\title{
Dynamic Emergence of Neuronal SYNCHRONY DURING KINDLING
}

\author{
A Dissertation \\ Presented to \\ the faculty of the School of Engineering and Applied Science \\ University of Virginia \\ in partial fulfillment \\ of the requirements for the Degree \\ of Doctor of Philosophy \\ by
}

Xin Ren

August

2013 
APPROVAL SHEET

The dissertation

is submitted in partial fulfillment of the requirements

for the degree of

Doctor of Philosophy

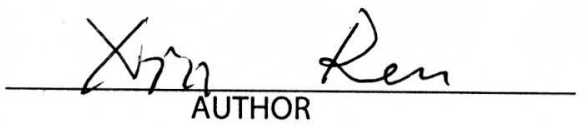

The dissertation has been read and approved by the examining committee:

\begin{tabular}{c} 
John L. Hudson \\
\hline $\begin{array}{c}\text { Advisor } \\
\text { Jaideep Kapur }\end{array}$ \\
\hline Giorgio Carta \\
\hline Howard Goodkin \\
\hline Michael Shirts \\
\hline
\end{tabular}

Accepted for the School of Engineering and Applied Science:

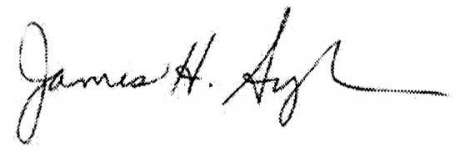

Dean, School of Engineering and Applied Science

August

2013 


\section{Abstract}

The hippocampus is involved in the generation and propagation of epileptic seizures. Although it has been stated that seizures are hypersynchronized activity of neurons, some studies showed desynchronization during seizure onsets. Most of the previous studies were conducted in brain slices. However, the structure of synchronization and propagation during hippocampal seizures in vivo is still undeveloped.

The purpose of this dissertation was to understand the dynamic properties of neuronal firing and quantify the synchrony of high frequency firing in both anesthetized and awake rats during epileptogenesis. A high-speed dynamic recording apparatus was constructed with a microelectrode array to record electrically evoked seizures. We found that as the epileptogenesis progresses, firing of neurons in CA1 region become more synchronized and the propagation is along the lamellar axis in CA1, but not the septotemporal axis. The synchrony of neurons was measured by using the following methods: cross correlation, theta phase synchronization and event synchronization. Cross correlation revealed that the firing pattern was highly correlated during evoked seizures along the lamellar axis but not the septotemporal. Both theta phase synchronization and event synchronization demonstrated that neuronal synchrony increased along the lamellar axis of CA1 pyramidal neurons as kindling progressed, while synchrony along the septotemporal axis remained at a 
relatively low level. Additionally, the theta phase distribution demonstrated that the firings of CA1 pyramidal cells became preferential for the negative peak of the theta oscillations as the seizure progresses. This was only true in the lamellar direction. Lastly, event synchronization shows that neuronal firings along the lamellar axis were more synchronized than those along the septotemporal axis.

The effects of two commonly prescribed antiepileptic drugs, phenytoin and levetiracetam, on seizure activity and neuronal synchronization in the electrically kindled model were examined. There was a marked decrease in synchronization and propagation after treatment with both phenytoin and levetiracetam. The preferred firing phase, which is around the negative peak of theta oscillation, was lost after both drug treatments.

In this dissertation, we discovered the temporal relationship between the structure of synchrony in CA1 and seizure severity. It can be used to help design a new deep brain stimulation algorithm to interrupt synchrony during epileptogenesis. 


\section{Acknowledgments}

I would like to thank my advisor, Professor Hudson, and my mentor, Professor Kapur, for their time, patience and guidance over the course of this dissertation.

Thank you to all the current and past members of the Hudson and Kapur lab groups for the research based and non-research based discussions and for assistance in my research. In particular, I would like to thank Craig Rusin, Sarah Johnson and Karen Blaha for taking the time to answer my questions, helping me with the rig, and making the days go faster. Also, many thanks to my lovely friends and family.

Last, but not least, I would like to thank my dear boyfriend, Samuel Kowalski, for his generous help, support and patience.

This work was funded through NIH and the Biomedical Innovation Fund/Ivy Foundation at UVA. 


\section{Contents}

1 Introduction $\quad 1$

2 Background 4

2.1 Hippocampal Seizures . . . . . . . . . . . . . 4

2.2 Microelectrode Array . . . . . . . . . . . . . . . . . 4

2.3 Electrical Kindling and Hippocampal Circuits . . . . . . . . . . 6

2.4 Seizure Propagation . . . . . . . . . . . . . 8

2.5 Synchronization Measurements . . . . . . . . . . . . . 9

2.6 Anti-Epileptic Drugs . . . . . . . . . . . . . . 13

2.7 Scope and Outline of this Dissertation . . . . . . . . . 14

3 Experimental Methods $\quad 15$

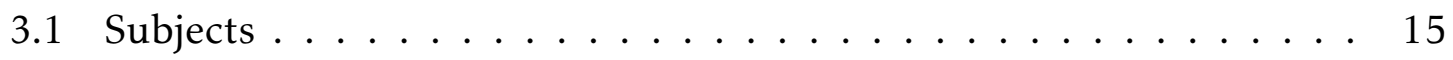

3.2 Electrode Design . . . . . . . . . . . . . . 15

3.3 Survival Surgery . . . . . . . . . . . . . . 16

3.4 Electrode Placements . . . . . . . . . . . . . . 18

3.5 Recording and Data Acquisition . . . . . . . . . . . . 19

3.6 Seizure induction ....................... 20

3.7 Anti-epileptic Drugs . . . . . . . . . . . . . . 21

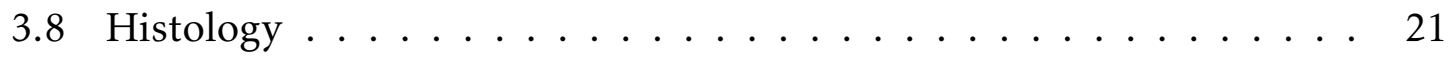


3.9 Synchronization and Propagation Measurement . . . . . . . . . 22

3.9 .1 Cross Correlation . . . . . . . . . . . . . . . 23

3.9.2 Theta Phase Synchronization . . . . . . . . . . 23

3.9.3 Event synchronization $\ldots \ldots \ldots \ldots \ldots \ldots$

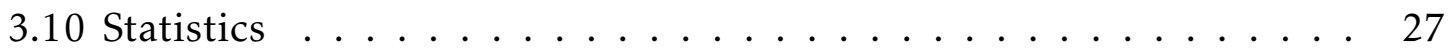

4 Synchronization along the CA1 Lamellar Axis in Anesthetized Rats 28

4.1 Microelectrode Array . . . . . . . . . . . . . . . 28

4.2 Electrically Evoked Seizures _ . . . . . . . . . . . 30

4.3 Synchronization . . . . . . . . . . . . . . . 31

4.3.1 Cross Correlation . . . . . . . . . . . . . . 33

4.3.2 Theta phase Synchronization . . . . . . . . . 34

4.3 .3 Event Synchronization . . . . . . . . . . . . . . 38

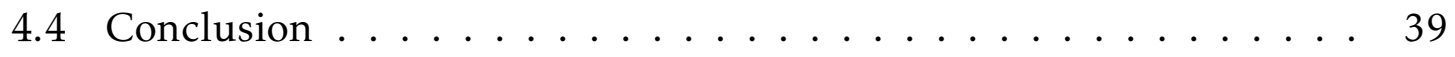

5 Synchronization along the CA1 Lamellar and Septotemporal Axes in Awake Rats 43

5.1 Evoked Seizures . . . . . . . . . . . . . . . . . . . 43

5.2 Behavioral Seizure Scores and After-discharge Duration . . . . . 45

5.3 High Frequency Firing $\ldots \ldots \ldots \ldots$

5.4 Spike Rate . . . . . . . . . . . . . . . . 47

5.5 Cross Correlation . . . . . . . . . . . . . . . . 52

5.6 Theta Phase Synchronization . . . . . . . . . . . . . . 52

5.6.1 Oriented Along the Lamellar Axis . . . . . . . . . . . . 52

5.6 .2 Oriented along the Septotemporal Axis . . . . . . . . . 57

5.7 Event Synchronization $\ldots \ldots \ldots \ldots \ldots$

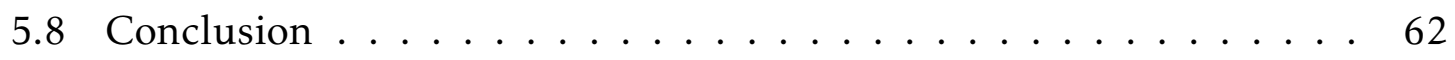


6 Synchronization during Anti-epileptic Drug Treatment 64

6.1 Phenytoin Treatment . . . . . . . . . . . . . 65

6.1.1 Behavioral Seizure Scores and After-discharge Duration . . . 65

6.1 .2 Spike Rate ... . . . . . . . . . . . 66

6.1.3 Theta Phase Synchronization ............ 67

6.1 .4 Event Synchronization . . . . . . . . . . 70

6.2 Levetiracetam Treatment . . . . . . . . . . . . . 71

6.2.1 Behavioral Seizure Scores and After-discharge Duration . . . 71

6.2 .2 Spike Rate ...................... 73

6.2.3 Theta Phase Synchronization ............ 73

6.2.4 Event Synchronization . . . . . . . . . . . 76

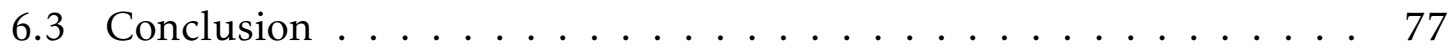

$\begin{array}{lll}7 & \text { Discussion } & 78\end{array}$

8 Future Work $\quad 83$

8.1 Neuronal Firing Patterns in the Dentate Gyrus and Hippocampal CA1 during Seizures .................... 83

8.2 Deep Brain Stimulation . . . . . . . . . . . . . . . 85

A MATLAB Code for EEG Data Analysis 86

A.1 Spike Detection . . . . . . . . . . . . . . 86

A.2 Theta Phase Synchronization . . . . . . . . . . . 88

A.2.1 Assign Theta Phase ............... . . . 88

A.2.2 Shannon Entropy . . . . . . . . . . . . . 91

A.3 Event Synchronization . . . . . . . . . . . . . . 92

B List of Publications Based on the Dissertation 97 
viii

\section{List of Figures}

2.1 Hippocampal Circuits and Formation . . . . . . . . . . . . 7

2.2 The Rat Hippocampus . . . . . . . . . . . . . . . . 10

3.1 Electrode Design . . . . . . . . . . . . . . 17

3.2 Electrode Placements and Data Acquisition . . . . . . . . . . 19

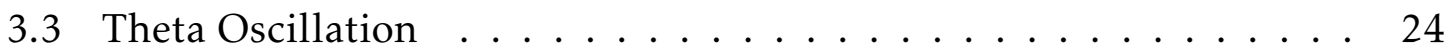

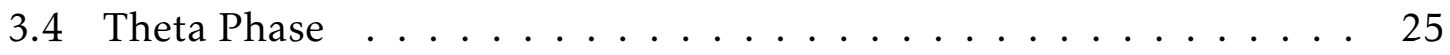

4.1 Microelectrode Array . . . . . . . . . . . . . . . . . . 29

4.2 EEG of An Evoked Seizure in Anesthetized Rats . . . . . . . . . . . . 31

4.3 ADD and the Number of Stimuli. . . . . . . . . . . . . . 32

4.4 High Frequency Firing during An Electrically Evoked Seizure . . . . 33

4.5 Correlation Coefficients Between Two Channels . . . . . . . . . . . . 35

4.6 Correlation Coefficients Between All Four Channels . . . . . . . . . 35

4.7 Temporal Relationship Between High Frequency Neuronal Firing and the Hippocampal Theta Oscillations . . . . . . . . . . 36

4.8 Theta Phase Distribution during An Evoked Seizure . . . . . . . 37

4.9 Distribution of Theta Phase Difference Between All Four Channels . 38

4.10 Schematic Plot of Propagation Direction in Hippocampal CA1 . . . 39

4.11 Schematic Plot of Propagation Pattern in Hippocampal CA1 . . . . . 40 
5.1 Artifact-free Electrically Kindled Seizure in An Awake Rat . . . . . 45

5.2 BSS and ADD During Kindling $\ldots \ldots \ldots \ldots \ldots$

5.3 High Frequency Firing Along the Lamellar Axis . . . . . . . . . . 48

5.4 High Frequency Firing Along the Septotemporal Axis . . . . . . 49

5.5 Spike Rate Distributions During Kindling . . . . . . . . . . . 50

5.6 Correlation Coefficients Between Two Channels Along Both the Lamellar and Septotemporal Axes . . . . . . . . . . . . . . . 51

5.7 Shannon Entropy Change During Kindling . . . . . . . . . . . . 53

5.8 Shannon Entropy of Stage 1 and Stage 5 Seizures Along the Lamellar

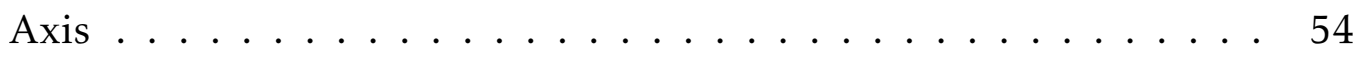

5.9 Theta Phase Distribution Along the Lamellar Axis . . . . . . . . 55

5.10 Theta Phase Difference Distributions Along the Lamellar Axis . . 56

5.11 Theta Phase Difference Distributions During Kindling . . . . . . . 56

5.12 Shannon Entropy During Kindling Along the Septotemporal Axis . 58

5.13 Shannon Entropy of Stage 1 and Stage 5 Seizures Along the Septotemporal Axis . . . . . . . . . . . . . . . . . . 59

5.14 Theta Phase Distribution Along the Septotemporal Axis . . . . . . 59

5.15 Theta Phase Difference Distributions Along the Septotemporal Axis 60

5.16 Event Synchronization Comparison Between the Lamellar and Septotemporal configurations $\ldots \ldots \ldots \ldots \ldots$. . . . . . 61

5.17 Event Synchronization During Kindling Along the Lamellar Axis . . 61

5.18 Event Synchronization During Kindling Along the Septotemporal Axis 62

6.1 BSS Before and After the Phenytoin Treatment $\ldots \ldots \ldots \ldots$

6.2 ADD Before and After the Phenytoin Treatment . . . . . . . . 66

6.3 Spike rate Before and After the Phenytoin Treatment . . . . . . . 67

6.4 Shannon Entropy Before and After the Phenytoin Treatment . . . . 68 
6.5 Theta Phase Distribution Before and After Phenytoin Treatment . . 69

6.6 Theta Phase Difference Distribution Before and Afte $r$ the Phenytoin

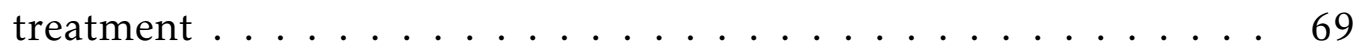

6.7 Event Synchronization Before and After the Phenytoin Treatment . 70

6.8 BSS Before and After the LEV treatment $\ldots \ldots \ldots \ldots \ldots \ldots$

6.9 ADD Before and After the LEV treatment . . . . . . . . 72

6.10 Spike rate Before and After the LEV Treatment $\ldots \ldots \ldots \ldots$

6.11 Shannon Entropy Before and After the LEV Treatment . . . . . . . 74

6.12 Theta Phase Distribution Before and After the LEV Treatment . . . 75

6.13 Theta Phase Difference Distribution Before and After the LEV Treat-

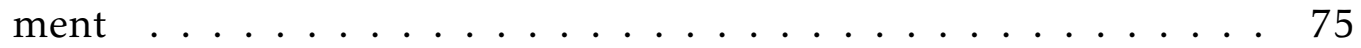

6.14 Event Synchronization Before and After the LEV Treatment . . . . 76 


\section{List of Tables}

3.1 The Racine five point behavioral seizure score . . . . . . . . . . . 21 


\section{Chapter 1}

\section{Introduction}

It has been hypothesized that epilepsy is a dynamic disease, characterized by a rapid transition from normal neural field firings to a hyper-synchronous, pathological state which is capable of spontaneously producing seizures [1]. Seizures are episodes of disturbed brain activity that cause changes in attention or behavior [2]. Recurrent epileptic seizures involving the hippocampus are endemic to temporal lobe epilepsy [3], and the propagation of seizures within the hippocampus is poorly understood. Studies have shown that even though the CA1 lamellar organization remains a useful concept for understanding hippocampal connectivity [3], both lamellar and septotemporal directions are involved in seizure propagation in the hippocampal CA1 $[4,5]$. However, most studies testing these hypotheses were conducted with brain slices, therefore hippocampal seizure propagation patterns in freely-moving animals is still undeveloped. While many studies have yielded deep insights into both synchronization and seizure propagation [6-9], there is still no consensus as to which quantitative measurement of synchrony is the best way of defining relationships within and between brain regions.

Kindling refers to the repeated administration of stimuli in order to elicit a 
heightened response from an experimental animal [10]. In the kindling model, seizures occur at the will of the investigator; thus this model ideally is suited for investigating the mechanisms of epilepsy [11]. This takes the form of periodically exposing the animal to stimulation trains in order to generate seizures. It is maintained until a certain threshold is reached (in our case, 3-6 successive stage 5 behavioral seizures), at which point the animal is considered to be fully kindled.

A microelectrode array was developed to record groups of neuronal activities in animal models of temporal lobe epilepsy during kindled seizures $[12,13]$. The experimental protocol used a common reference for the microelectrode array instead of recording differentially.

Different linear and nonlinear synchronization analyses have been previously used with limited success. In recent studies, different phase synchronization methods were used to determine the firing patterns in seizures $[14,15]$. However, the application of phase synchronization to electroencephalography (EEG) signals is fraught with difficulty [16]. Holmes et al. studied spontaneous hippocampal firing and its relationship to theta rhythms in rats after status epilepticus [9]. Grassberger et al. used event synchronization (ES), which is not based on phase analysis, to quantify the degree to which events are synchronized between two time series, such as rat EEG signals [17]. In this study, we used two nonlinear synchronization methods, theta phase synchronization and event synchronization, together with linear cross correlation to quantify neuronal synchronization and propagation.

Various antiepileptic drugs (AEDs) are widely used and are primarily intended to treat epileptic seizures [17]. The ultimate goal of antiepileptic drugs is to eliminate seizures while minimizing side effects $[18,19]$. Intensive studies have been done in vitro to discern the mechanisms by which different AED's function in vitro [18-20]. However, it is still unknown how, or even if, antiepileptic drugs change neuronal 
synchrony in vivo.

The aim of this project was to understand the dynamic properties of neuronal firings and to evaluate the synchrony between simultaneously-recorded neuronal activities. The experiments are divided into three parts. First, we examined the dynamic properties of neuronal firing and developed methods to quantify synchrony between neuronal firings. Second, neuronal synchronization and propagation patterns, in both the lamellar and septotemporal directions of the hippocampus CA1 region, were studied in freely moving rats in an electrical kindling model of temporal lobe epilepsy. Third, two antiepileptic drugs were tested for their effects on neuronal synchrony and propagation along the lamellar axis in CA1 region.

In this dissertation, we extended the existing body of research in several ways. Firstly, seizure propagation was examined in the hippocampus of anesthetized and freely moving rats, rather than in brain slices. Secondly, seizure synchronization and propagation were investigated in both the lamellar and septotemporal directions of the CA1 region in order to create a two dimensional synchrony and propagation structure in the hippocampus. Third, two antiepileptic drugs were tested for their effects on neuronal synchronization and propagation during kindling. 


\section{Chapter 2}

\section{Background}

\subsection{Hippocampal Seizures}

The hippocampus belongs to the limbic system and plays an important role in the consolidation of information from short-term memory to long-term memory, as well as spatial navigation [21]. The majority of neurons in the hippocampal layers are densely packed pyramidal cells and granule neurons. Hippocampal seizures are believed to be synchronized tonic-clonic bursts of action potentials riding on a prolonged wave of depolarization through a network of neurons [21-23]. Seizures appear to arise from a perversion of normal physiological characteristics of neurons and neuronal networks [24, 25].

\subsection{Microelectrode Array}

For decades, epileptologists have been relied on macroscopic electroencephalogram (EEG) recordings from the scalp (non-invasive) to produce global and local measurements of scalp potentials [26-31]. However, hippocampal seizures some- 
times cannot be satisfactorily recorded with non-invasive techniques [32]. Depth electrodes are capable of recording signals from buried cortex region, such as the hippocampus, which can be hard to detect with other types of electodes [33]. They have been used to find dysfunctional brain regions and onset of seizures [34]. With the development of depth recording, a new technique called microelectrode array is used for extracellular neural recording [6, 35-39]. In this dissertation, a microelectrode array was incorporated into our recording system to investigate the dynamics of neuronal firings during the development of electrically evoked seizures in vivo. The microelectrode array simultaneously recorded from several local groups of cells neighboring each other during evoked seizures. The spatial scale of our experiment was limited to a local hippocampal CA1 cell layer. This technique helps us analyze the complicated circuit.

Seizures are composed of high frequency firing superimposed over the low frequency waveforms [21]. In this study, we were interested in the high frequency firing which constitutes the bursts during seizures. These bursts represent neuronal firings from local fields. In order to capture the fast firings, the sampling rate (Fs) must be large enough to ensure that multiple samples are recorded during the most rapid extracellular voltage swings. Empirically, measured extracellular waveforms show significant frequency content at values up to about $8 \mathrm{kHz}$ [40]. According to Nyquist sampling theorem, the minimum sampling rate to record $8 \mathrm{kHz}$ is about 16 $\mathrm{kHz}$. Sanchez et al. showed a pronounced increase in high frequency oscillations (like bursts) during seizures when the sampling rate was $24 \mathrm{kHz}$ [41]. Therefore we used a sampling rate of $25 \mathrm{KHz}$ for our experiments. 


\subsection{Electrical Kindling and Hippocampal Circuits}

Kindling was originally discovered by Graham Goddard [3]. Kindling refers to a dynamic process during which prolonged afterdischarges are induced by repeated administration of electrical stimulation [42]. Conventional kindling, i.e. electrical stimulation of the amygdala for 1 second once daily, induces secondary after-discharges in the hippocampus. However, this process is time and labor consuming. In contrast, rapid kindling, i.e. electrical stimulation of the CA3 region for 10 seconds every 30 minutes 12 times per-day, is characterized by delivering long stimulus trains at short intervals to elicit a reliable series of seizures [43]. The repetition of the stimulus every other day leads to electrographic and behavioral advancements, hence the fully kindled state (more details in 3.1.2).

The electrically kindled rat model is widely used in studies of hippocampal seizures [44]. With electrically kindled rats, the focus of the seizure is controlled by the placement of the stimulating electrode, and the occurrence of seizures is controlled by the investigator [3]. As kindling is much less neurologically insulting than the status epilepticus models, electrical kindling is ideal for studying the process of epileptogenesis and testing the effectiveness of antiepileptic drugs on temporal lobe epilepsy [45]. Electrical kindling involves both the pyramidal cells of the CA1 region and granule cells in the dentate gyrus [42]. The hippocampus is a key structure in kindling and limbic systemic epileptogenesis, and different regions in the hippocampus serve different roles. The hippocampal CA1 region is known for its innate capacity for generating seizures, and the CA3 region is especially suited to the development of interictal spikes, which are highly correlated with the occurrence of spontaneous seizures [46].

The main pathway in functional hippocampus anatomy is the trisynaptic pathway 


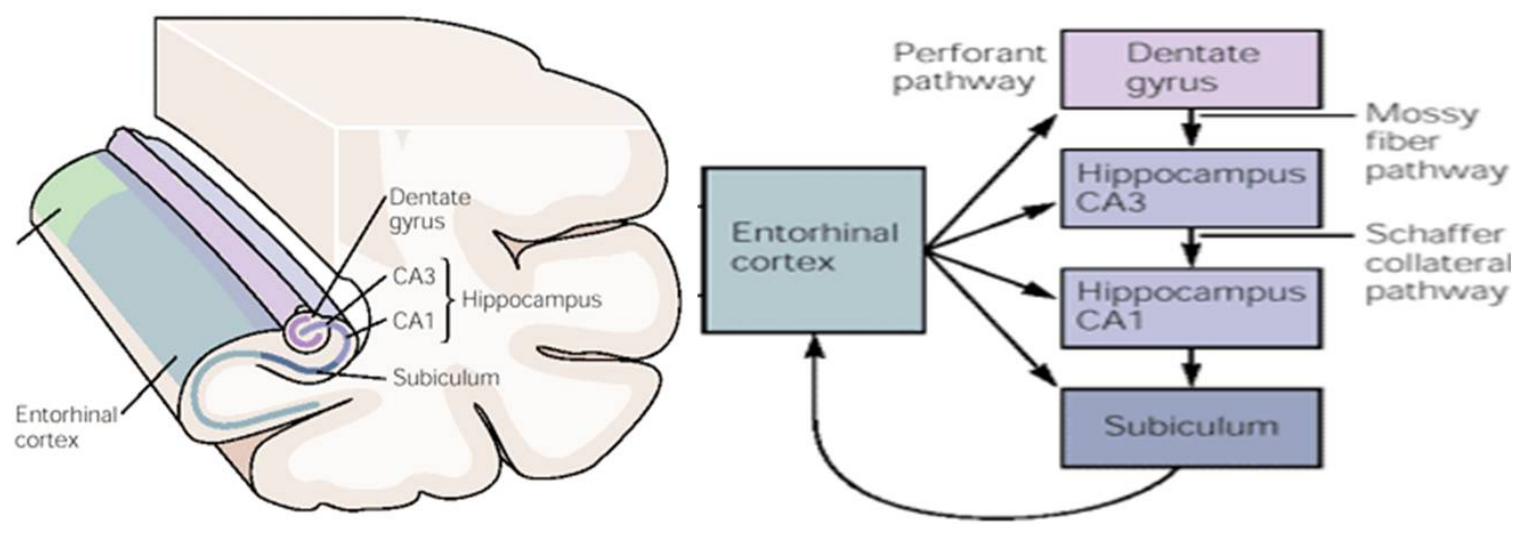

Figure 2.1: (Left) The anatomical organization of the hippocampal formation; (Right) Functional anatomy of hippocampal circuits in epileptogenesis. Figure adapted from Schultz[49].

(see Figure 2.1), which involves three consecutive excitatory synapses (Perforant pathway, Mossy fiber pathway and Schaffer collateral pathway) [46]. The first synapse is the perforant pathway, between the entorhinal cortex neuron axons and the granule cells of the dentate gyrus. The second is the mossy fibers, from granule cells and terminating on CA3 cells. The third is the Schaeffer collateral, from CA3 pyramidal cells and ending on CA1 pyramidal cells.

Even though kindling does not cause a large loss of neurons in the hippocampus [47], it induces neuronal circuit reorganization (mossy fibers sprouting from preexisting axons within the hilus of the dentate gyrus and along the dorsal-ventral hippocampal axis) and a variety of cellular alterations [45]. The hippocampal circuitry damage, which is caused by kindled seizures, induces memory dysfunction and deficits. Therefore, kindling might be potentially relevant to mechanisms of learning and memory formation [48]. 


\subsection{Seizure Propagation}

The generation and propagation of epileptiform activity in the hippocampus has been studied extensively. The lamellar hypothesis was first introduced in the early 1970s by Andersen et.al [50] from studies of anesthetized rabbits. They hypothesized that the hippocampal cells were activated in a lamellar fashion and that the principal excitatory pathways of the hippocampus were organized in a lamellar fashion. In Andersen's study, a lamellar, a group of cells in a transverse band, was used to represent a functional unit of the hippocampus (Figure 2.2). The lamellar hypothesis had tremendous influence on the study of hippocampal information processing. However, other researchers demonstrated that the connection between CA3 neurons and CA1 pyramidal cells, the Schaffer collateral, was heavily branched in a markedly diverging pattern [51]. Amaral and Witter [4] proposed that the major projection from CA3 to CA1 would be more divergent than is consistent with a strict interpretation of the lamellar hypothesis. From the physiological and anatomical data, they concluded that it is more reasonable to consider the hippocampus as a three-dimensional cortical region with important information processing taking place in both the transverse and longitudinal axes. Recently, with the development of intact in vitro hippocampal preparation, the propagation of intra-hippocampal epileptiform activity in both the longitudinal and transverse directions has been studied $[1,5,52]$. These studies concluded that the epileptiform wave propagation pattern is three dimensional. Andersen et al. [52] further proposed that, in spite of the fact that the Schaffer collateral originates and radiates from CA3 neurons within a wide fan-shaped area, the amplitude of the action potential was largest in transverse band across the CA1.

Most of the previous work on the propagation of such epileptiform activity has 
been done with brain slices $[50,53]$. With the development of multi-electrode arrays, many studies have been done to map firing patterns during seizures in vitro. It has been shown that ictal discharges at seizure onset may fail to recruit adjacent territories in vitro [54]. There are some limitations in the slice studies, such as neuronal connectivity might be interrupted by slicing the tissue. Seizure propagation along the lamellar and septotemporal directions has not been tested in freely moving animals. Quantitative methods are needed to measure propagation.

\subsection{Synchronization Measurements}

In 1954, Penfield and Jasper [56] first reported that seizures were characterized of hypersynchronized neuronal firings. Ever since then, numerous experiments have been done to prove this excessive synchronization of activity in a large population of neurons [57-59]. It is yet unclear if seizures can be simply described as a hypersynchronized state, or if there are more complex mechanisms underlying seizure generation, maintenance and propagation. Several studies suggest that seizures are very complex activities, and may even involve both synchronized and desynchronized neuronal dynamics [2, 58, 60-64]. Schevon et al. [54] suggested that the core area within the seizure onset zone had intense, hypersynchronous tonic/clonic ictal patterns in human seizures; while the surrounding area showed heterogeneous and unstructured firings.

In a review article published earlier in 2013, they showed that synchronization in seizures depends on several factors, such as the spatial scale, which signals were processed and the definition of synchrony [65]. Existing methods to measure synchronization include cross-correlation, mutual information, spectrum-based coherence, nonlinear interdependence, and phase synchronization [66-75]. The 

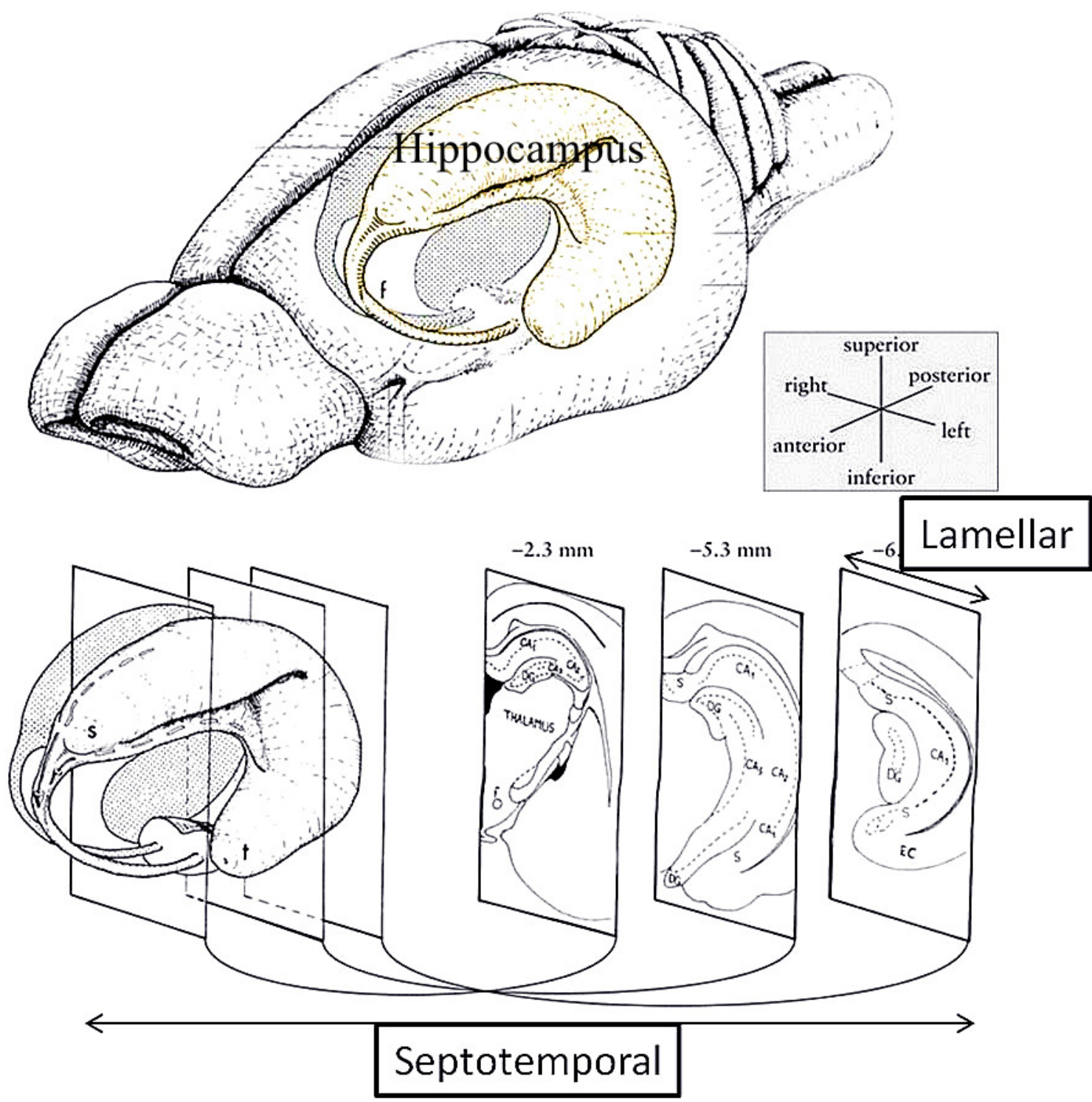

Figure 2.2: The rat hippocampus is indicated in yellow in the upper figure. The lower part shows the hippocampus in both the lamellar and septotemporal directions. Figure adapted from Amaral and Witter [55]. 
methods that give useful information about the interdependency of the signals are chosen based on experience of the researches. This indicates that the definition of synchronization needs to be clarified before application. Generally speaking, synchronization measurements fall into two categories, linear and non-linear. Linear correlation evaluates a linear relationship, while non-linear synchronization reveals a non-linear functional relationship between the dynamics of two systems [76]. The most commonly used linear measurement of synchrony is cross correlation [77-85]. Cross correlation studies the similarity of two waveforms; it has been used to calculate the correlation between local feild potentials [66]. Two most commonly used non-linear methods are time delays and phase synchronization. In recent years, several phase synchronization methods have been proposed and applied successfully to different types of data $[17,86]$. Event synchronization, which is based on time delays, has been used as a tool to determine seizure severity, as well as the underlying network dynamics of seizures [17]. Schiff et al. [73] explained that non-linear synchronization is a better candidate for complex neuronal systems, such as seizures, than linear methods. However, the comparison of which method (linear or non-linear) is better at detecting synchronization in epilepsy remains an active and open area.

In this study, we were particularly interested in three different methods for the measurement of synchrony (cross correlation, theta phase synchronization and event synchronization). Since the neurons do not fire periodically like chemical oscillators, the actual application of the phase model to neuronal firing data is vastly more difficult. As we are interested in seizure activity in the hippocampus, an underlying clock for our phase model was needed. Theta oscillations are a prominent $4-10 \mathrm{~Hz}$ rhythm in the hippocampal local field potential [87], and it play an important role in modulating spiking and act like the internal clock of 
the hippocampal circuit[88-90]. Theta phase synchronization was designed to use theta oscillations to investigate the synchrony of hippocampal high frequency firing. Theta phase increases linearly from the trough of one spike in the theta oscillations to the next. The firings during the seizures are assigned with theta phase values, which are between 0 and $2 \pi$. A phase distribution is created for each seizure. Shannon entropy is used to calculate the order of a phase distribution, which is then used to describe the synchrony of the system. If the temporal relationship between the neuronal firings and theta rhythm is random, then phase distribution is uniformly distributed. However, if the firings have a preferential relationship with the theta rhythm, the phase distribution is unimodal, bimodal or multimodal. For the simplest cases, if the neurons fire strictly at a specific theta phase, the neurons are said to be phase locked [91]. For more complicated cases, if the neurons oscillate faster than the frequency of the theta rhythm, a progressive phase precession of firing is observed [92]. Due to this phase precession phenomenon, two cells with partially overlapping fields will fire at specific but different phases of the ongoing theta oscillation.

The definition of event synchronization is less stringent, where synchronization only implies that defined events are occurring simultaneously, or near simultaneously, in time. Action potentials are defined as discrete events in time and synchronization is calculated from the near-simultaneous occurrence of action potentials within a small time window. The advantage of event synchronization is that it is not affected by frequency changes during bursting activity. 


\subsection{Anti-Epileptic Drugs}

In the past decade, the mechanism and behavioral effects of various antiepileptic drugs (AEDs) have been studied intensely. We were particularly interested in two drugs, phenytoin (PHT) and levetiracetam (LEV).

Phenytoin was introduced in the 1930s [93]. It is a very effective anticonvulsant drug in controlling a wide variety of seizure disorders [94]. Phenytoin obstructs recurrent action potentials by blocking voltage-dependent sodium channels [20, 94]. In the electrical kindling model, phenytoin is a powerful suppressor of limbic behavioral seizures and afterdischarges that are elicited by brief stimulation trains [46].

Levetiracetam (LEV) is a relatively new antiseizure drug. It is an effective and well tolerated adjunctive therapy for refractory focal seizures $[95,96]$. LEV has multiple mechanisms of action $[97,98]$. Relevant to this research, it has been shown to bind to synaptic vesicle protein 2 (SV2) [99]. Loss of SV2 reduces the release probability of action potentials, which means that synaptic strength is decreased specifically during high frequency firing, like bursting activity [100]. It has been shown in some in vivo kindling models to reduce the severity and duration of seizures $[101,102]$.

Although, previous works have revealed changes in the behavioral seizure scores and duration of after-discharges after phenytoin and levetiracetam treatments [46, 101-103], the changes in neuronal synchronization with anti-epileptic treatment have not been fully investigated and are poorly understood. 


\subsection{Scope and Outline of this Dissertation}

The goal of this dissertation was to examine synchronization in the CA1 cell layer in an electrical kindling model of temporal lobe epilepsy in rats.

Chapter 3 examined a technique called the microelectrode array, which was used to record groups of neuronal firings during electrically evoked seizures in anesthetized rats.

Chapter 4 studied synchronization in both lamellar and septotemporal directions in hippocampus CA1 region in awake rats. The dynamic synchronization and propagation of neuronal firings along both the lamellar and septotemporal axes during evoked seizures were analyzed by the same methods. Distinct firing patterns were seen along the lamellar axis of hippocampal CA1 region, but not along the septotemporal axis. Both theta synchronization and event synchronization showed that as kindling progressed, synchronization increased in the lamellar direction.

Chapter 5 demonstrated the effects of two conventional AEDs, with different mechanisms, on synchronization. Both of the AEDs attenuated the synchronization of neuronal firings. These changes were statistically significant changes in the synchronization level after both AEDs treatments. 


\section{Chapter 3}

\section{Experimental Methods}

\subsection{Subjects}

All experiments were performed in accordance with the National Institutes of Health's Guide for the Care and Use of Laboratory Animals, and were approved by University of Virginia Animal Care and Use Committee (ACUC).

Adult male Sprague-Dawley rats, weight 250-300g, were used for all experiments. They were housed two per cage on a light/dark cycle in a temperature-controlled room with access to water.

\subsection{Electrode Design}

Bipolar electrodes were constructed from two equal length $500 \mu \mathrm{m}$ stainless-steel coated wires (A-M System). The wires were twisted and cut at an angle to prevent short-circuiting between the tips.

The microelectrode array was fabricated from 8 strands of $50 \mu \mathrm{m}$ nichrome coated wires (A-M System). 
The procedure used to fabricate the electrode array is as follows: All wires were lined up with the tool, and then glued together (See Figure 3.1). Several hours were allowed for the glue to harden. The tips were cut transversely with sharp scissors so that the tips were at the same surface level. Adjacent electrodes were separated by a spacing wire (50 $\mu \mathrm{m}$ nichrome coated wire). Before implantation, the electrodes tips were examined under microscope to make sure no superglue was present at the exposed surface. The impedance of each electrode was typically 25-40 KHz. Figure 3.1(bottom) shows the dimensions of the wire and resulting electrode configuration. The common reference was a stainless steel wire placed in the skull or scalp, and the potential difference was measured with respect to the common reference.

Each electrode was attached to a lightweight head-stage and connected to the input of a customized operational amplifier. The outputs of the operational amplifier were connected to the analog inputs of a recording amplifier.

\subsection{Survival Surgery}

During the electrode placement, the rats were anesthetized with urethane (1000 $\mathrm{mg} / \mathrm{kg}$ ) or isoflurane, placed in a Kopf stereotaxic frame, and maintained on a heating pad $\left(37 \pm 0.5^{\circ} \mathrm{C}\right)$. The surgical area was shaved, cleaned with povidoneiodine, and prepared for aseptic surgery using the following protocol:

1. A single incision was made along the midline of the scalp to expose the skull, and the skin was propped open. Small hemostats were used to hold the skin open. The periosteum was removed from the scull by scalpel blade.

2. Four small holes were drilled for screws, which were in contact with dura mater. Stainless screws were inserted into the skull to stabilize the electrodes. 


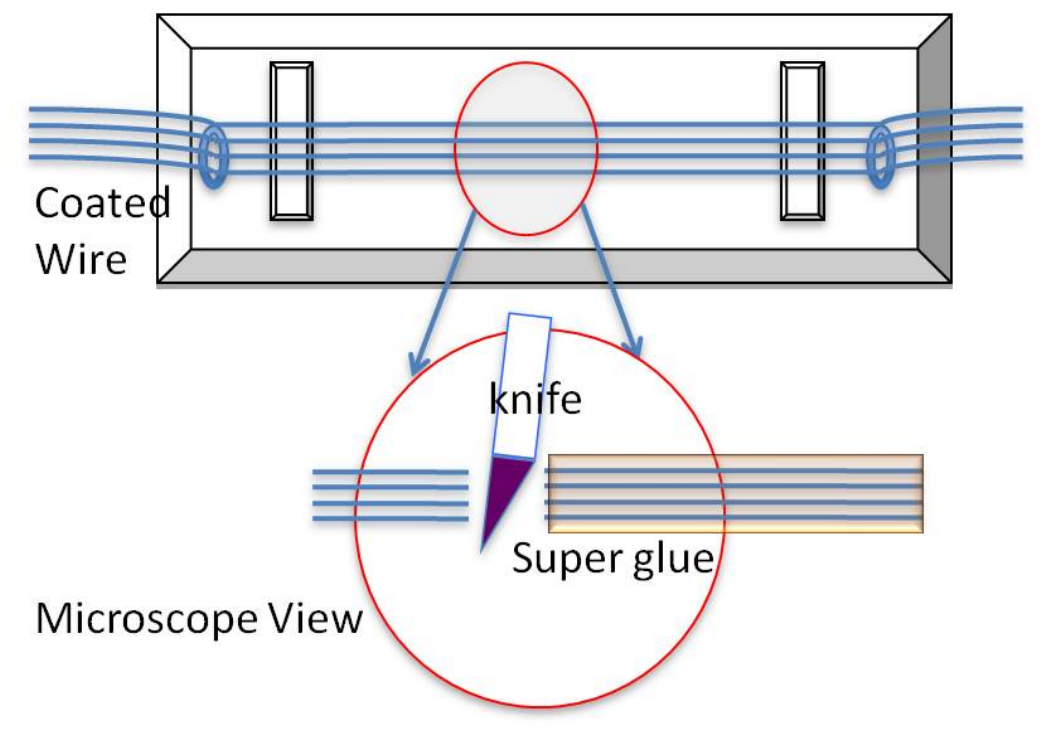

$0.5 \mathrm{~mm}$
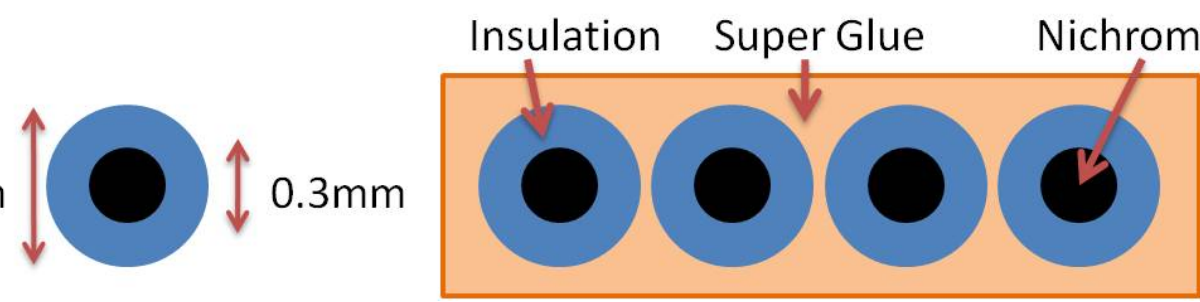

Figure 3.1: (Top) Drawing of the electrode construction. The tips were cut transversely with a sharp knife and examined under microscope to make sure no superglue was attached on the cross section surface. (Bottom) Dimensions of the outer and inner diameters of the wire.

3. The cranium were drilled to accommodate the electrode arrays. The underlying dura mater was pierced to accommodate electrode insertion without producing compression of the brain. The skull was dabbed with saline until all the bone dust was removed.

4. A bipolar stainless-steel stimulating electrode was placed in the CA3 at the coordinates described in the next section. The microelectrode array was then implanted, and its final coordinates tuned to the appropriate site via the method described in Electrode Placements.

5. A layer of dental cranioplastic was used to cement the electrodes, screws 
and cranium to each other, in order to prevent any shifting. The electrodes outgoing connector was embedded in cranioplastic as well, in order to form a mechanically stable cap.

6. The scalp was restored around the cap using a single staple at the rear of the incision.

Recording began immediately after electrode implantation in the anesthetized rat studies. In the freely moving studies, the animal received ketoprofen, $10 \mathrm{mg} / \mathrm{kg}$, i.p for analgesia following the surgeries and were allowed 7-10 days recovery after surgeries before being used in an experiment.

\subsection{Electrode Placements}

A bipolar electrode (500 $\mu \mathrm{m}$ coated stainless-steel wires, A-M Systems) was stereotactically implanted for stimulation in hippocampal CA3 region $(\mathrm{A},-3 \mathrm{~mm} ; \mathrm{L}$, $3.5 \mathrm{~mm}$; V, 2.5-3.0 mm below dura). A microelectrode array was implanted in the contralateral hippocampal CA1 region (A, $3.0 \mathrm{~mm}$; L, $2.0 \mathrm{~mm}$; V,2-2.5 mm below dura) along the transverse or longitudinal hippocampal axis for field recording (see Figure 3.2 (Left)). The microelectrode array was fabricated from 8 strands of $50 \mu \mathrm{m}$ coated nichrome wires (10 $\mu \mathrm{m}$ coating, A-M System). The recording sites are 100 $\mu \mathrm{m}$ apart from center to center. Responses to perforant pathway stimulation were used to find the optimal microelectrode array position to record CA1 pyramidal cell firing. Electrode positions were confirmed via histological examination, as described below. A coated stainless steel wire (1 mm, A-M System) was placed in the skull or scalpel to serve as a common reference electrode. 


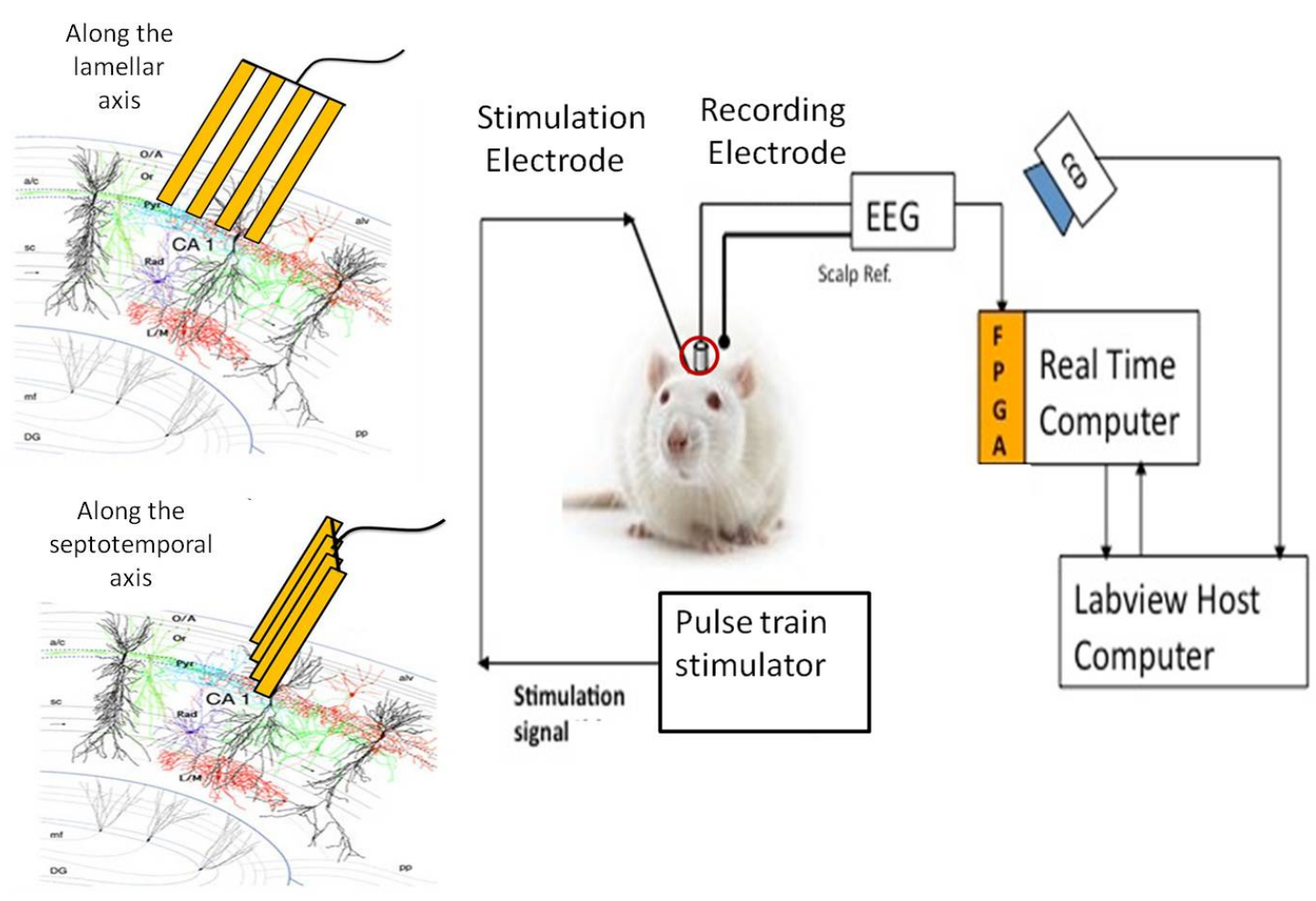

Figure 3.2: (Left)Schematic figures of the microelectrode array placement in the hippocampal CA1 cell layer. (Right)Diagram of the dynamic recording apparatus. Stimulation from the pulse train stimulator is applied to the animal. EEG signal is recorded and fed into the real time computer through an amplifier.

\subsection{Recording and Data Acquisition}

The animals were enclosed in a metal mesh cage that was connected to the ground of the system in order to decrease electrical noise. The signals from the microelectrode array were buffered with an operational amplifier attached to a lightweight head-set (gain=1). The operational amplifiers were connected to 4 channel amplifiers (A-M systems, model 1700) via flexible cables, through a rotating commutator. The analog output port of the amplifier was connected to an analog input channel of the DAQ system. The DAQ system consisted of two computers, a real-time controller and a LabVIEW-based host computer. The controller utilized 
a high-speed FPGA processor to acquire data at a $25 \mathrm{KHz}$ sampling rate, yielding a resolution of $40 \mu$ s. The LabVIEW-based host computer was used for the postprocessing and visualization of the experimental data (see Figure 3.2 (Right)). The recording was continuous before, during, and after the electrically kindled seizures. All channels of the microelectrode array were recorded from $1 \mathrm{~Hz}$ to $1 \mathrm{KHz}$ and later on passed through a high-pass filter of $300 \mathrm{~Hz}$ for off-line high frequency firing analysis.

\subsection{Seizure induction}

For anesthetized rats, stimuli trains $(10 \mathrm{~s}, 50 \mathrm{~Hz}, 0.5 \mathrm{~ms}$ biphasic pulses) were delivered at 15 minute intervals, for a total of 12 times. Recordings were obtained continuously before, during, and after electrically evoked seizures.

A rapid kindling protocol was used for freely moving rats. According to the protocol, stimuli trains (10 seconds, $50 \mathrm{~Hz}, 1 \mathrm{~ms}$ biphasic pulses) were delivered at 30 minutes intervals 10 to 12 times per day. The rats were stimulated every other day to avoid artificial after-discharge threshold elevation. The kindling threshold was determined for each animal by checking for the presence of an after-discharge (ADD) and was varied from $400 \mu \mathrm{A}$ to $800 \mu \mathrm{A}$. To determine the after-discharge threshold, we started with a low intensity $(400 \mu \mathrm{A})$. Intensity was then increased until a seizure was trigged. ADDs were measured from the end of the stimulus to the termination of the after-discharge. The five point behavioral seizure score (BSS) system was utilized to rank the degree of seizure severity (see Table 3.1) [104]. The animals were required to demonstrate 3 to 6 Stage 5 seizures in succession to be considered fully kindled. Recordings were obtained in the same manner as the anesthetized animals. 
Table 3.1: The Racine five point behavioral seizure score

Stage Behavior

$1 \quad$ Mouth and facial movement

2 Head nodding

3 Forelimb clonus

$4 \quad$ Rearing with forelimb clonus

$5 \quad$ Rearing and falling with forelimb clonus (generalized motor convulsions)

\subsection{Anti-epileptic Drugs}

Before the treatment phase, it was verified that all the animals were fully kindled, means that at least three kindled stage 5 seizures had occurred consecutively. Four animals received 1 dose of phenytoin $75 \mathrm{mg} / \mathrm{kg}$, and another four animals received 1 dose of levetiracetam $125 \mathrm{mg} / \mathrm{kg}$. Drug doses were based on the basis of prior studies on the behavioral effects of these drugs [53-55].

Phenytoin (Sigma Chemical Co., St. Louis, MO) was dissolved in Dimethyl sulfoxide (Sigma Chemical Co., St. Louis, MO). Levetiracetam (Sigma Chemical Co., St. Louis, $\mathrm{MO}$ ) was dissolved in $0.9 \%$ sodium chloride solution. Both drugs were given i.p. 10 minutes after the fully kindled seizure and 30 minutes before the next stimulation.

\subsection{Histology}

After the recording was completed, the animals were sacrificed via $\mathrm{CO} 2$ asphyxiation. The brains were removed and stored in a solution of $1 \%$ potassium ferricyanide and $4 \%$ paraformaldehyde in $0.1 \mathrm{M}$ phosphate buffer at $4{ }^{\circ} \mathrm{C}$ for at least one night. Potassium ferricyanide was used because it reacts with residual iron from the electrodes to form prussian blue, thus aiding in the confirmation of 
electrode placement. Brains were then frozen and sectioned perpendicular to the septotemporal axis (on a sliding microtome) to a thickness of $40 \mu \mathrm{m}$. The electrode locations appeared as azure spots on the slices. The location of the recording sites was confirmed for all animals used in this study.

\subsection{Synchronization and Propagation Measurement}

In neuronal synchronization, firing events from different neurons occur at the same time, or at least within a short time frame. Synchronization of the network of pyramidal neurons can rapidly occur because of extensive positive feedback that is mediated by recurrent axon collateral in the CA3 region. Synchronization in the CA1 region occurs when inhibition is suppressed. Pyramidal neurons have intrinsic bursting properties and can rapidly synchronize and propagate bursts, resulting in seizures [22]. This makes the CA1 region a good target for the study of synchronization of neuronal firings. In this study, we implant the microelectrode array in the CA1 region. The microelectrode array enables simultaneous recording in multiple sites, which can be analyzed for their functional interactions, such as synchronization and propagation [7]. Neurons are connected to each other through sprawling and interwoven networks. Many studies have shed light on synchronization and seizure propagation within such neuronal systems $[6-9,54]$. However, there is no consensus as to which quantitative measurement of synchrony is the best way to define relationships within brain regions. Existing methods include: cross-correlation, mutual information, spectrum-based coherence, nonlinear interdependence, and phase synchronization $[6-9,54]$. In this study, various techniques, including phase synchronization and event synchronization, are compared in order to find the most robust measurement of synchrony. 


\subsubsection{Cross Correlation}

Conventional approaches to identify the synchronization of firings are based on the use of cross correlation techniques, applied to groups of neuronal activities. In this dissertation, cross correlation represents a time-averaged correlation among the firing events of the participating neurons. Cross correlation is a standard method of estimating the degree to which two time series are correlated. Consider two time series $x(i)$ and $y(i)$ where $i=0,1,2 \ldots N$. The cross correlation coefficient $r$ is defined as

$$
r=\frac{\sum_{i}\left(x_{i}-m_{x}\right)\left(y_{i}-m_{y}\right)}{\sqrt{\sum_{i}\left(x_{i}-m_{x}\right)^{2}} \sqrt{\sum_{i}\left(y_{i}-m_{y}\right)^{2}}}
$$

where $m_{x}, m_{y}$ are the means of the corresponding time series, and $\mathrm{N}$ is the number of samples in the time series. The analysis is based on field potentials that are produced by synchronous activity in groups of neurons close to each recording electrode of the microelectrode array. Correlation coefficients for two channels of firings were calculated across the duration of the seizure. The correlation between two groups of firing was measured by the value of coefficients.

\subsubsection{Theta Phase Synchronization}

The theta rhythm, a hippocampal network pattern in the $4-10 \mathrm{~Hz}$ frequency band, is often recognized as the defining electrophysiological signature of hippocampal activity during temporal coding/decoding of active neuron ensembles. The extracellular currents underlying theta waves are generated mainly by the entorhinal input, CA3 Schaffer collateral, and voltage-dependent Ca2+ currents in pyramidal cell dendrites [105]. It is important to reveal the relationship between synaptic activity (as reflected by field theta oscillation) and local high frequency neuronal firings 


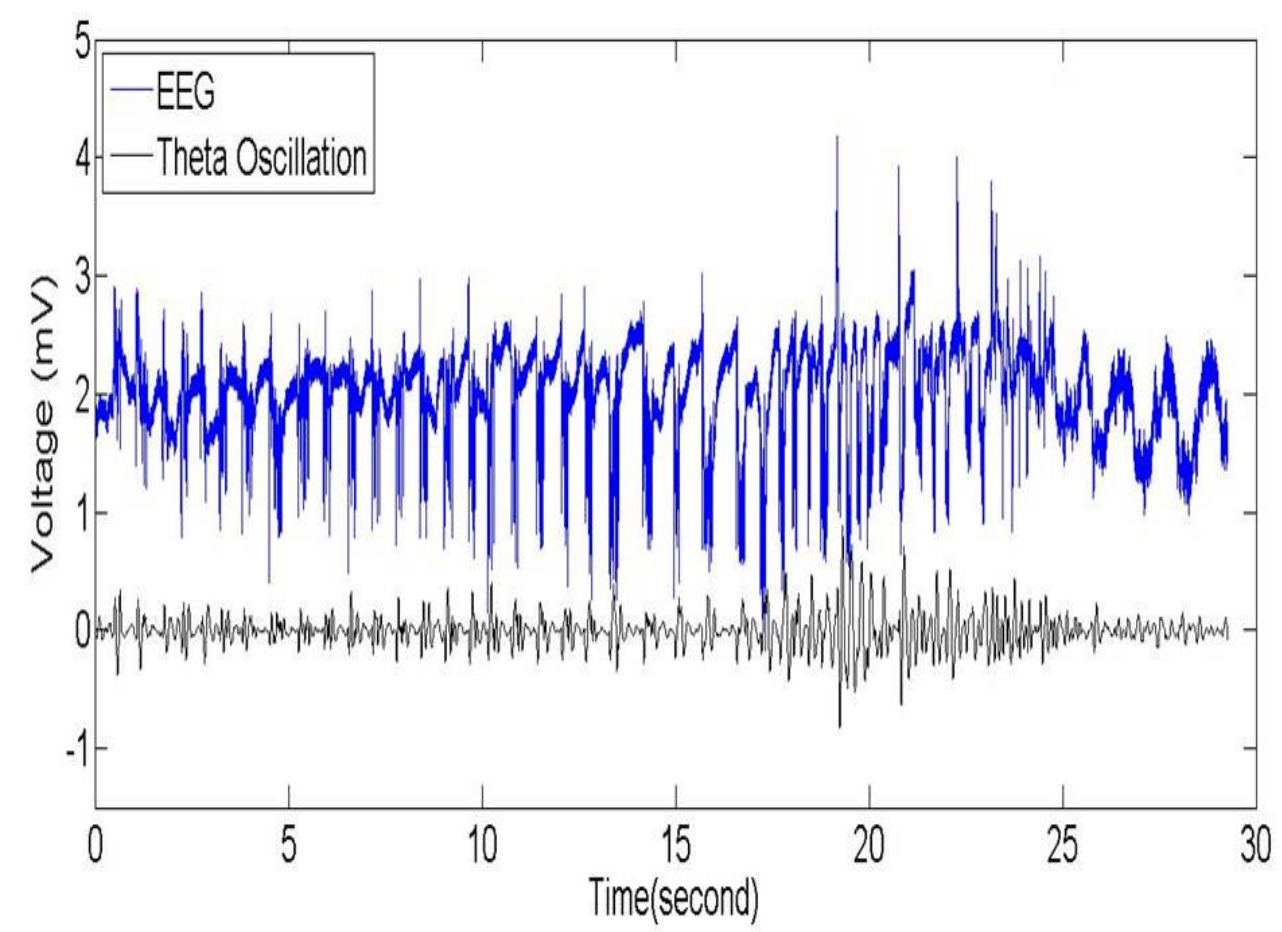

Figure 3.3: The top trace is EEG signal and the bottom trace is theta oscillation.

$[105,106]$.

The EEG signal was band-pass filtered between $4-10 \mathrm{~Hz}$ to extract the theta oscillation. The theta cycle is defined as the time between two adjacent negative peaks in the theta oscillation (Figure 3.3). Theta phase is linearly increased from 0 to $2 \pi$ from the peak of one negative peak to the next. For this definition of synchronization, the change in the phase is of interest, and amplitude is largely irrelevant.

In the case of neuronal firings, the peaks of action potentials were assigned with theta phase values that were determined by the firing time within that phase window (Figure 3.4).The propagation pattern in hippocampus CA1 region was evaluated by calculating the phase differences between groups of firing. The phase difference can be any value from 0 to $2 \pi$. 


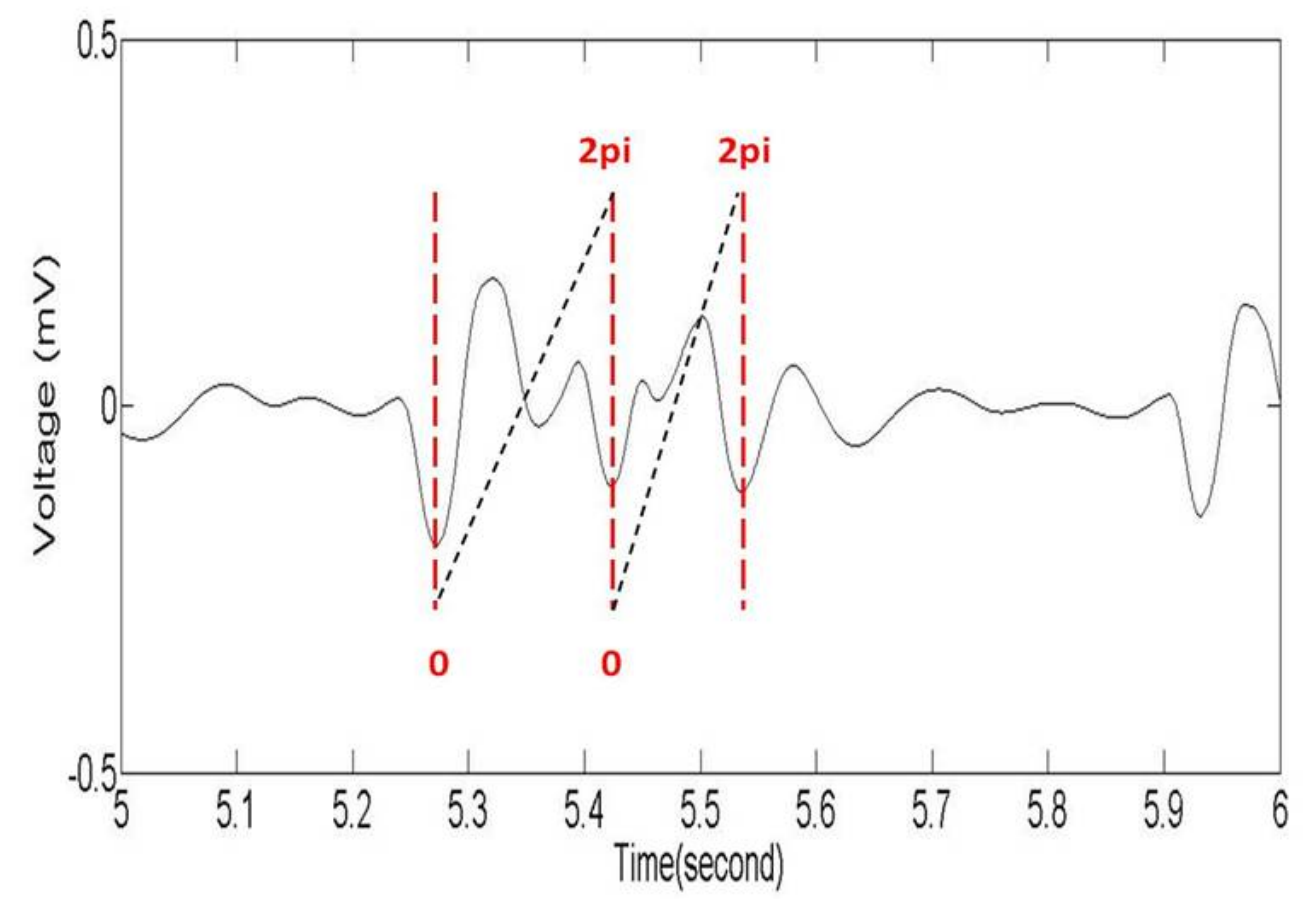

Figure 3.4: The waveform is the theta oscillation. The red dotted lines indicate the negative peaks of the theta oscillation. The theta cycle is defined as the time between two negative peaks. Each time point is assigned with a theta phase value from 0 to $2 \pi$ via linear interpolation.

The Shannon entropy is a standard measurement of the order state of a sequence of data (in this case, the theta phase data during a seizure). It quantifies the degree of skew in the distribution. Therefore, Shannon entropy was used to quantify the order of theta phase distributions.

The phase synchronization between two oscillating elements was calculated by Shannon entropy $(S)$ :

$$
S=-\sum_{i} p_{i} \ln p_{i}
$$

with $S_{\max }=\ln M$, where $M$ is the number of bins and $p_{i}$ is the probability of the phase difference being in the $i$-th bin. For complete desynchronization, the value is zero. 


\subsubsection{Event synchronization}

This measurement of synchrony is less stringent, it only implies that defined events are occurring simultaneously or near simultaneously in time. Event synchronization (ES) is used to quantify the degree of synchronization of events in two time series, such as rat EEG signals [17]. ES quantifies the number of times an event occurs in two time series within a small window and does not require the notion of phase. Given recordings from two electrodes of the microelectrode array, time series $x$ and $y$, the event times are defined as $t_{i}^{x}$ and $t_{j}^{y}$. Action potentials that occur in both signals within a time interval $\pm \tau$ are considered to be synchronized. The number of times an event appears in $\mathrm{x}$ shortly after it appears in $\mathrm{y}$ is defined as

$$
c^{\tau}(x \mid y)=\sum_{i=1}^{n_{x}} \sum_{j=1}^{n_{y}} J_{i j}^{\tau}
$$

with

$$
J_{i j}^{\tau}= \begin{cases}1, & \text { if } 0<t_{i}^{x}-t_{j}^{y} \leq \tau \\ 1 / 2, & \text { if } t_{i}^{x}=t_{j}^{y} \\ 0, & \text { else }\end{cases}
$$

where $n_{x}$ is the number of action potentials in neuron $x$, and $n_{y}$ is the number of action potentials for neuron $y$, with $i=1, \ldots, n_{x}$ and $j=1, \ldots, n_{y}$. The values of $c^{\tau}(x \mid y)$ and $c^{\tau}(y \mid x)$ are then combined symmetrically $(Q)$ and antisymmetrically $(q)$ :

$$
Q_{\tau}=\frac{c^{\tau}(y \mid x)+c^{\tau}(x \mid y)}{\sqrt{n_{x} n_{y}}}
$$

where $Q_{\tau}$ is the strength of the ES. $Q_{\tau}$ varies from 0 to 1 with 0 being no synchronized events and 1 being completely synchronized events. 


\subsection{Statistics}

The minimum level for determining significance was $\mathrm{p}<0.05$ for all statistical tests performed. Different numbers of stars $(*, * *$, and $* * *)$ represent the levels of statistical significance. $*$ denotes $\mathrm{p}<0.05 ; * *$ denotes $\mathrm{p}<0.01$; and $* * *$ denotes $\mathrm{p}$ $<0.001$. Data was reported as mean \pm SD unless noted otherwise. The statistical tests used were the Student's t-test (MATLAB), ANOVA (MATLAB) and the Kruskalwallis test (MATLAB) as noted in the text and figures. Post-hoc multicomparison tests were done using the Tukey method (MATLAB). The Kolmogorov-Smirnov test (MATLAB) was used to test whether the difference in spike rate during different epochs was significant. 


\section{Chapter 4}

\section{Synchronization along the CA1 \\ Lamellar Axis in Anesthetized Rats}

The purpose of this study was to test the effectiveness of the microelectrode array at recording neuronal firings during electrically evoked seizures in anesthetized rats. Additionally, we used three measurements of synchrony to understand the dynamic properties of neuronal firings during seizures.

\subsection{Microelectrode Array}

To test this technique, a microelectrode array, which consists of a linear array of four electrodes, was implanted in hippocampus CA1 in depth. This design was based on the theory that extracellular field potentials are local current sinks, or sources, that are generated by the collective action potentials of many neurons [108]. A negative wave corresponds to a current sink, which is caused by positive charges entering cells through postsynaptic glutamate receptors, while a positive wave is generated by the current that leaves the cell (at the cell body) $[109,110]$. 


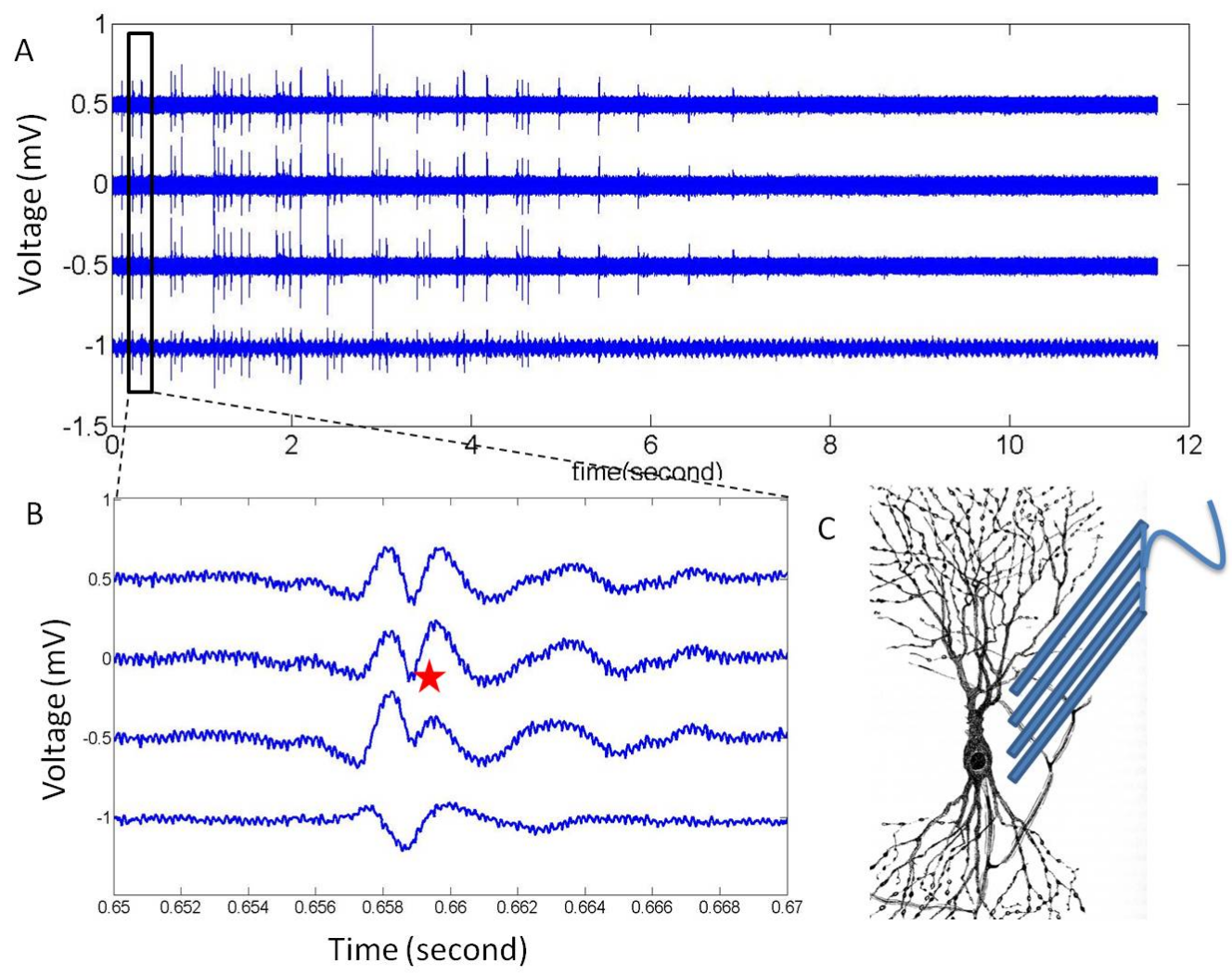

Figure 4.1: Waveform information is necessary for electrode placement. (A) Four channels of band-pass filtered data (300-4000Hz): An electrically evoked seizure was immediately recorded after 10 s of stimulation. (B) Waveform of a single spike recorded by the microelectrode array. (C) Schematic figure of the microelectrode array placement along the somatodendritic axis. The figure was adapted from frontalcortex.com [107]

As shown in Figure $4.1 \mathrm{C}$, the microelectrode array was placed in a vertical manner along the somatodendritic axis along the hippocampal CA1 cell layer. The top trace shows a seizure recorded by a microelectrode array. The field potentials recorded at various positions along the soma and dendritic tree are shown on the bottom. The waveform of the spike varied as a function of distance from the cell body. The population of spikes (star) was negative when recording from the cell body layer. This observation supports the assumption that the waveform of a single spike can be quite different depending upon the recording site. Also, the fact that 
the waveform changes with electrode advancement along the somatodendritic axis can be used as a guide for the electrode placements. The electrode array was placed in the cell body layer for following experiments.

\subsection{Electrically Evoked Seizures}

For anesthetized rats, firing activity was rarely seen in baseline EEG data. After several hours of kindling, varying with the individual rat $(n=6)$ and its response to anesthesia, recurrent seizures were observed. The first recording started after 2 hours of kindling. EEG was recorded at $25 \mathrm{KHz}$ by a microelectrode array in hippocampal CA1 along the lamellar axis. As shown in Figure 4.2, a 10s stimulation train was followed by 21 seconds of bursting activity. The duration of this bursting activity is called after-discharge duration (ADD). When the synchronous spiking died out, the EEG signal returned to the baseline amplitude.

Among 72 seizures recorded in six rats, the average ADD increased slightly as additional stimuli were delivered (see Figure 4.3). However, there was a slight increase of ADD over the time. There are several reasons why we did not observe a rapid increase in the ADD. Firstly, since the rats were under anesthesia, the kindling threshold varied substantially from rat to rat. This means that several rounds of testing stimulus were required to reliably evoked seizures. Secondly, we started to record after repeated seizures were observed, which about 1 to 2 hours into kindling was. Some of the rats generated relatively stable and consistent seizures after only a few exposures to the stimulus; while others were comparatively hard to kindle. The ADD was found to vary considerably from rat to rat, despite using the same kindling protocol. This was not surprising because the ADD is dependent upon the metabolic state of the neurons, which varies between rats. In general, 

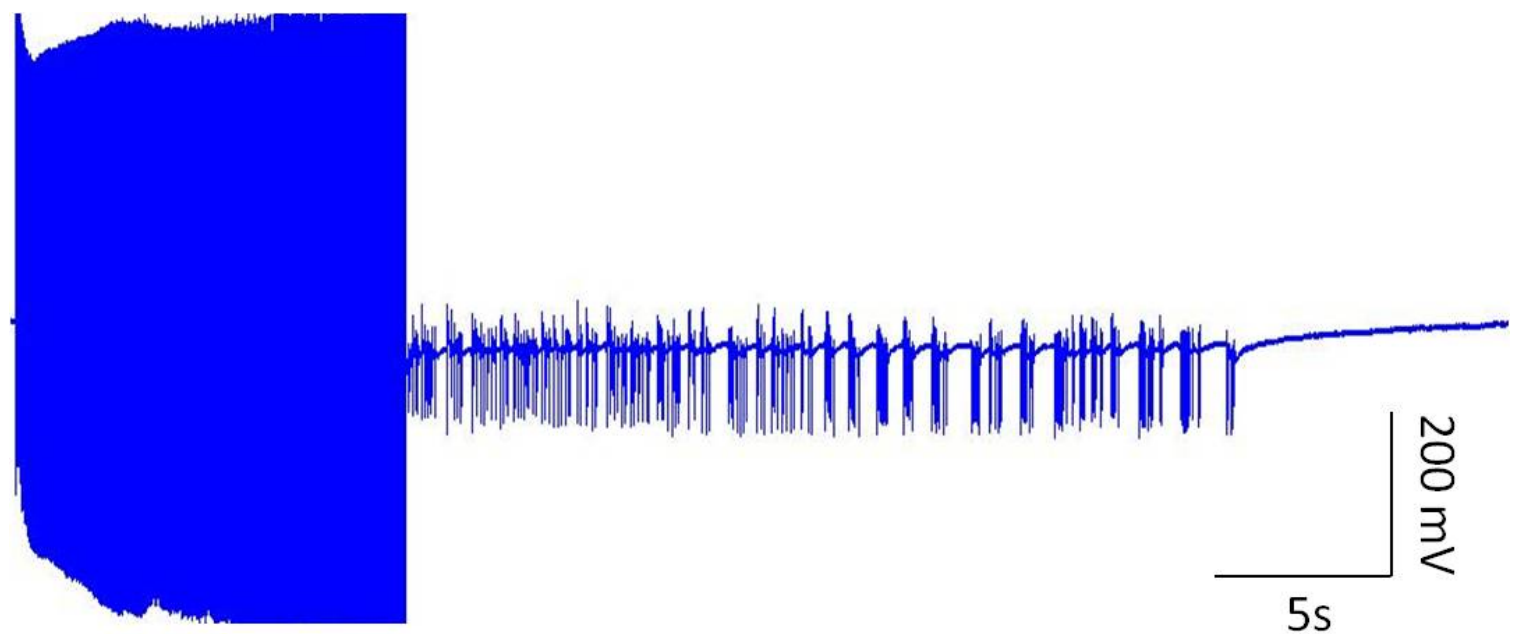

$5 s$

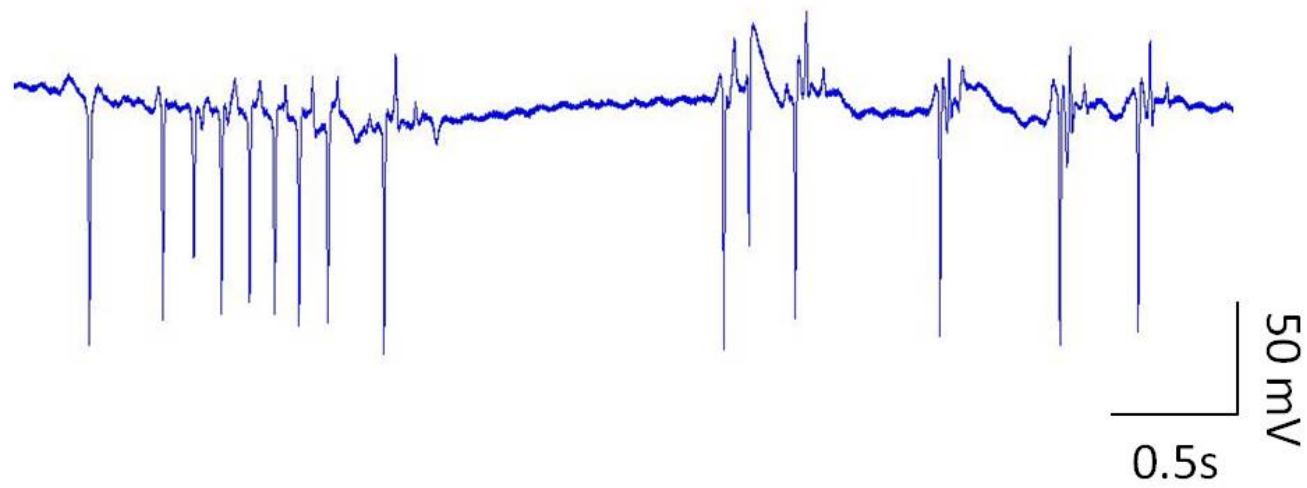

Figure 4.2: EEG of an evoked seizure in anesthetized rats (Top) EEG recording: A seizure recorded in the anesthetized rat. (Bottom) Expanded segment of the top trace. The bursting activity has a characteristic spike-and-wave shape. A high amplitude fast wave (spiking) was followed by a much lower amplitude slow wave.

the stimulation protocol successfully generated seizures whose ADD progressively lengthened with recurrent exposures to the stimulus.

\subsection{Synchronization}

Seizures are believed to represent synchronized neuronal activity. The most relevant features to quantify synchrony are phase and frequency. As for our syn- 


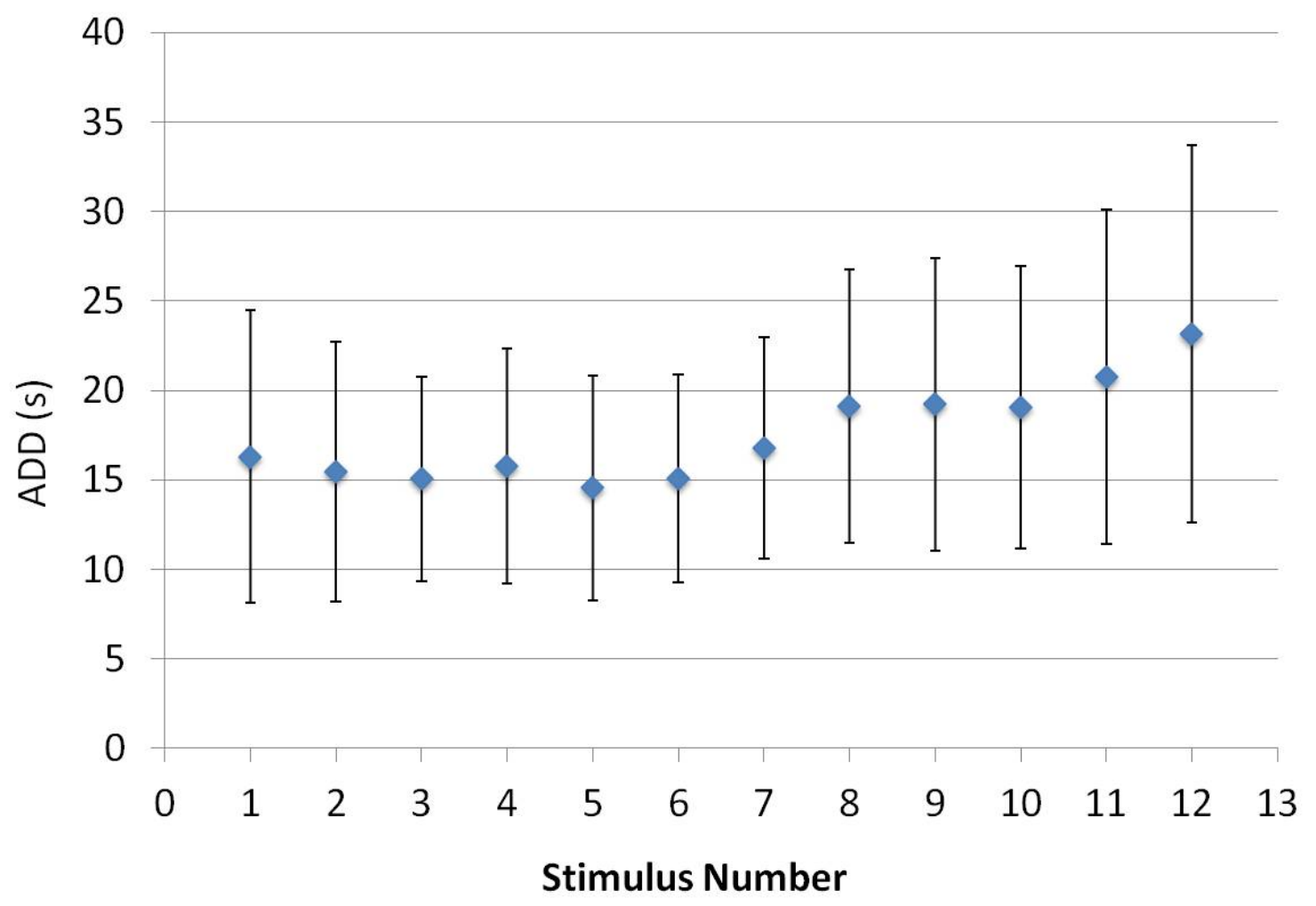

Figure 4.3: ADD increased over a number of stimuli.

chronization analysis, we were interested in the high frequency components during seizures. In order to measure these features, the raw signal was first band-pass filtered between $4-10 \mathrm{~Hz}$ to extract theta oscillations. The microelectrode array signals were passed through a band-pass filter between 300 to $4000 \mathrm{~Hz}$ for high frequency firing analysis (Figure 4.4). Expanded segments of a single spike are shown in the bottom; the waveforms of three firing events are on the same time scale. Similar waveforms were observed in all channels. During the early and middle phases of seizures, the waveform of a given spike displayed small variations between the four channels. By the end of a seizure, the amplitude of the firings was smaller, and more waveform variations were seen in different channels. The differences in amplitude and waveform observed from different channels suggest that the four closely spaced electrodes record from different groups of neurons. The 

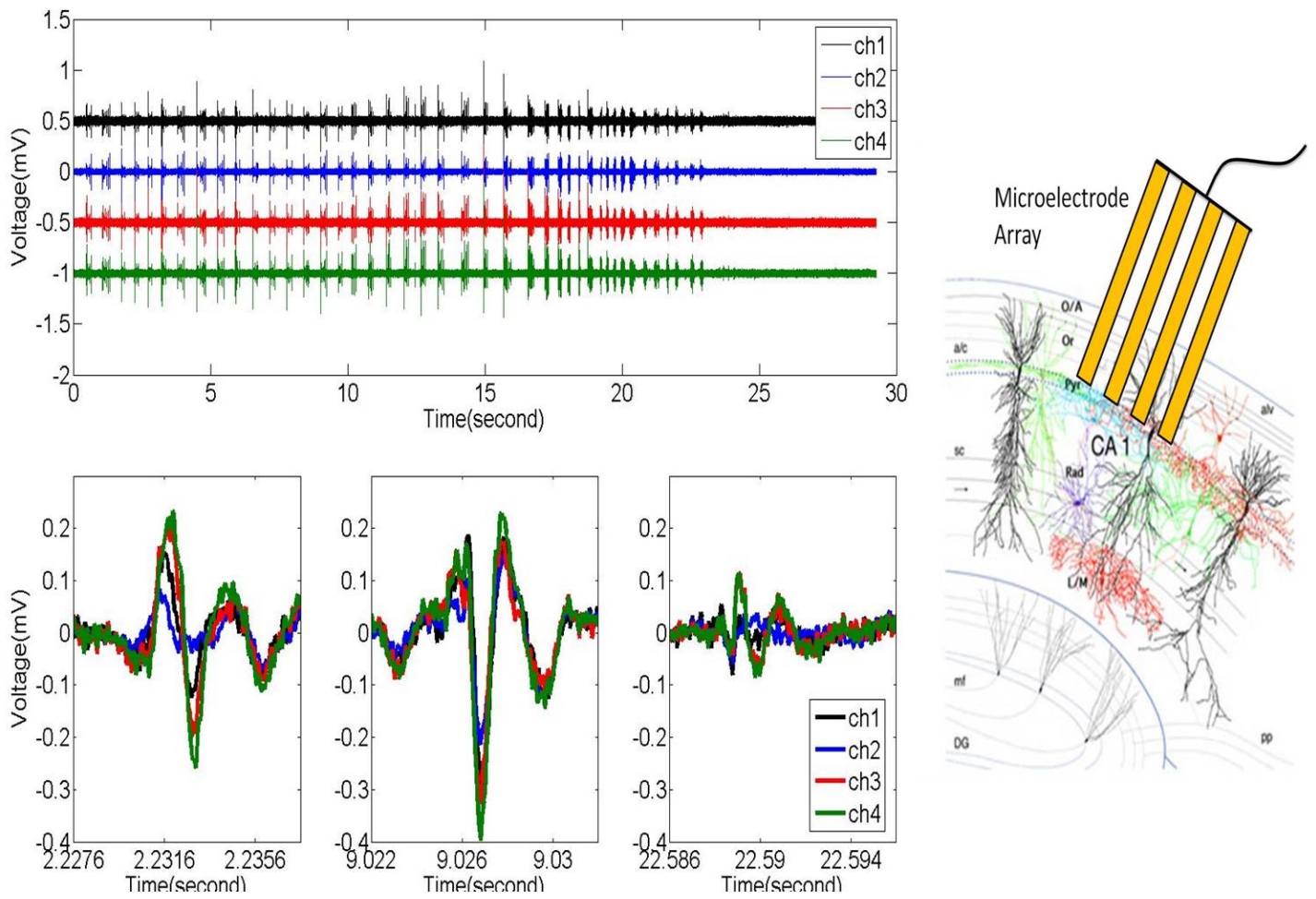

Figure 4.4: High frequency firing during an electrically evoked seizure in an anesthetized rat. (Left top) An electrically evoked seizure was recorded by the microelectrode array. All channels were filtered between 300 and 4000 $\mathrm{Hz}$. The 10 seconds of stimulation is not included in this figure. The EEG recording began immediately after stimulation. (Left Bottom) Expanded view of the top trace. Waveforms did not change much until the end of the seizure. (Right) Schematic figure of the microelectrode array placement along the hippocampal CA1 lamellar axis.

amplitude difference also suggests that different numbers of neurons are recorded by each electrode.

\subsubsection{Cross Correlation}

To start the analysis, correlation coefficients were calculated between every two channels of the recording which was filtered between 300 and $4000 \mathrm{~Hz}$. Cross correlation coefficients were plotted as a function of time (See Figure 4.5). The correlation coefficients started out at 0.61 , fluctuated in the range of 0.61 to 0.82 then 
dropped to 0.53 at the end of the seizure. Figure 4.6 shows the cross correlogram of pairs of channels during an evoked seizure. The mean correlation coefficients between channels varied from 0.66 to 0.92 . The cross correlation coefficients increased from a relatively low value and deviated around the mean during the middle phase of the seizure. For 5 out of the 6 pairs, the correlation coefficients attenuated towards the end of the seizure, which indicates that the correlation between the two time series became disrupted. Cross correlation successfully demonstrates how the correlation level changes during a single seizure. Additionally, it reveals that the correlation level varies as a function of the distance between the recording sites. Pair 1-2, 2-3, 3-4 were next to each other, hence they were more correlated than other pairs. Pair 1-4 was the furthest apart out of all pairs, and consequently channel 1 and channel 4 were the least correlated. The differences between different pairs' correlation coefficients also lessened as the seizure carried on. This suggests that the firings become more synchronized as the seizure continued within the area local to the microelectrode array.

\subsubsection{Theta phase Synchronization}

The temporal relationship between neuronal firings and theta oscillations was investigated to determine the structure of synchrony and propagation in the CA1 region. Theta oscillations function as a unique solution to the temporal segregates and links neuronal assemblies. The temporal relationship of high frequency neuronal firing to the hippocampal theta rhythm in electrically evoked seizures is illustrated in Figure 4.7. Different color marks in the figure correspond to peaks of the firing events in all four channels. The timing of the peaks varied from channel to channel. Some peaks only appeared in certain time series, which indicates the neurons seen by each electrode are different, but have some overlap. As mentioned in section 3.9, 

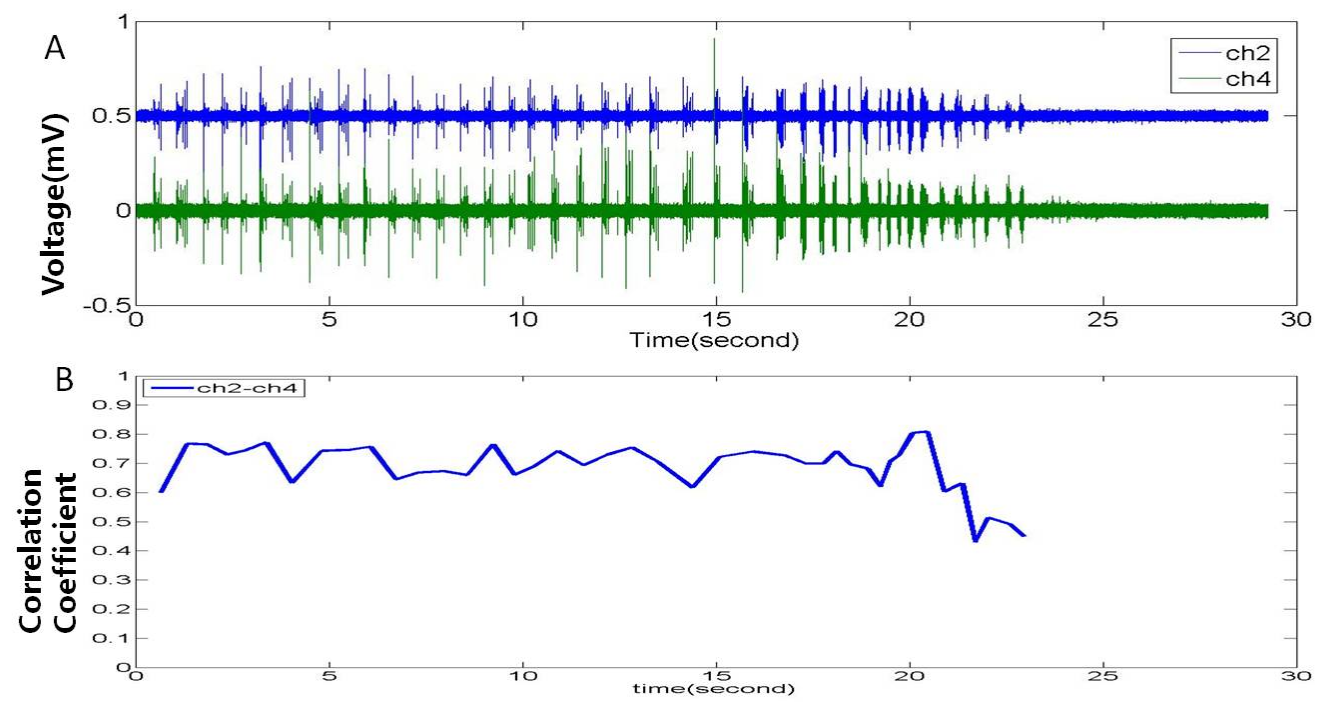

Figure 4.5: An example of the correlation profile between two channels of the microelectrode array recording during a seizure in an anesthetized rat.(A) Two channels of band-pass filtered data (300-4000 Hz) (B) Correlation coefficients between the two channels are plotted with time.

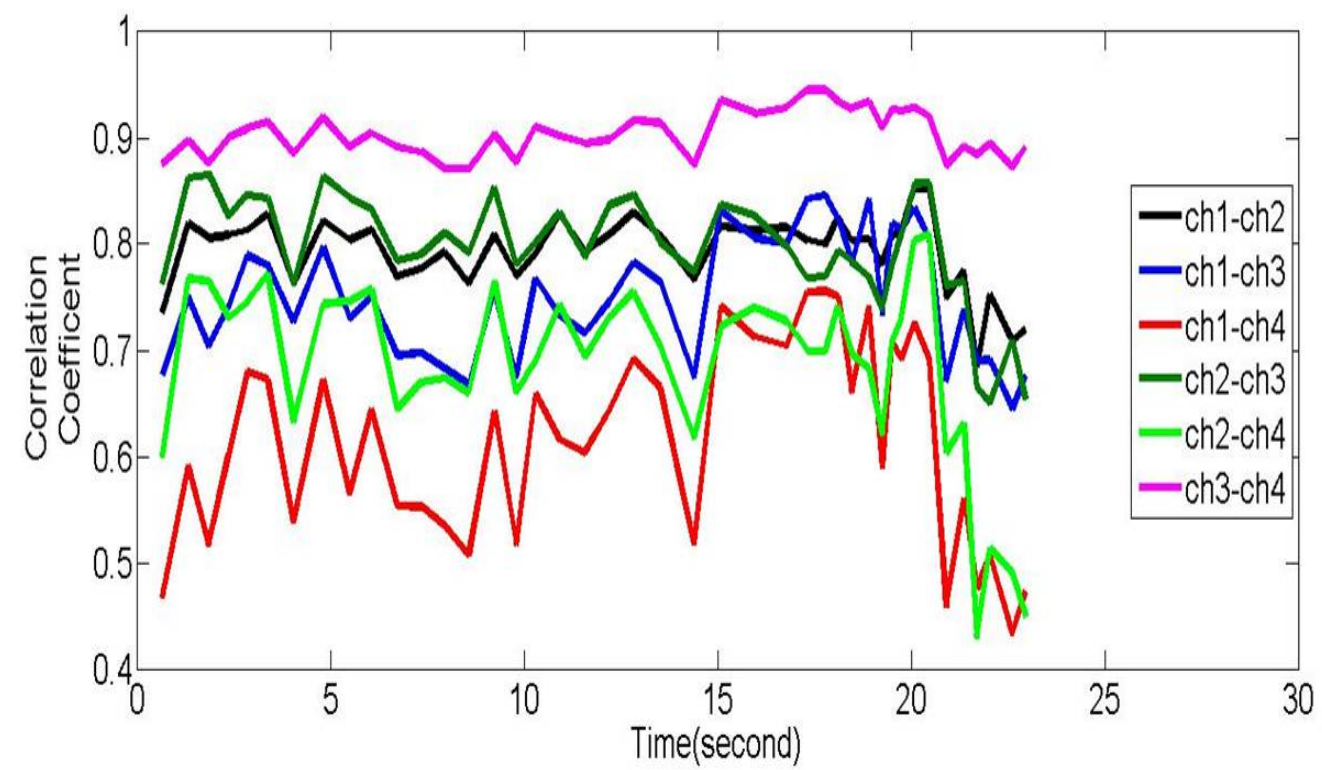

Figure 4.6: An example of the correlation profile between all channels of the microelectrode array recording during a seizure in an anesthetized rat. 


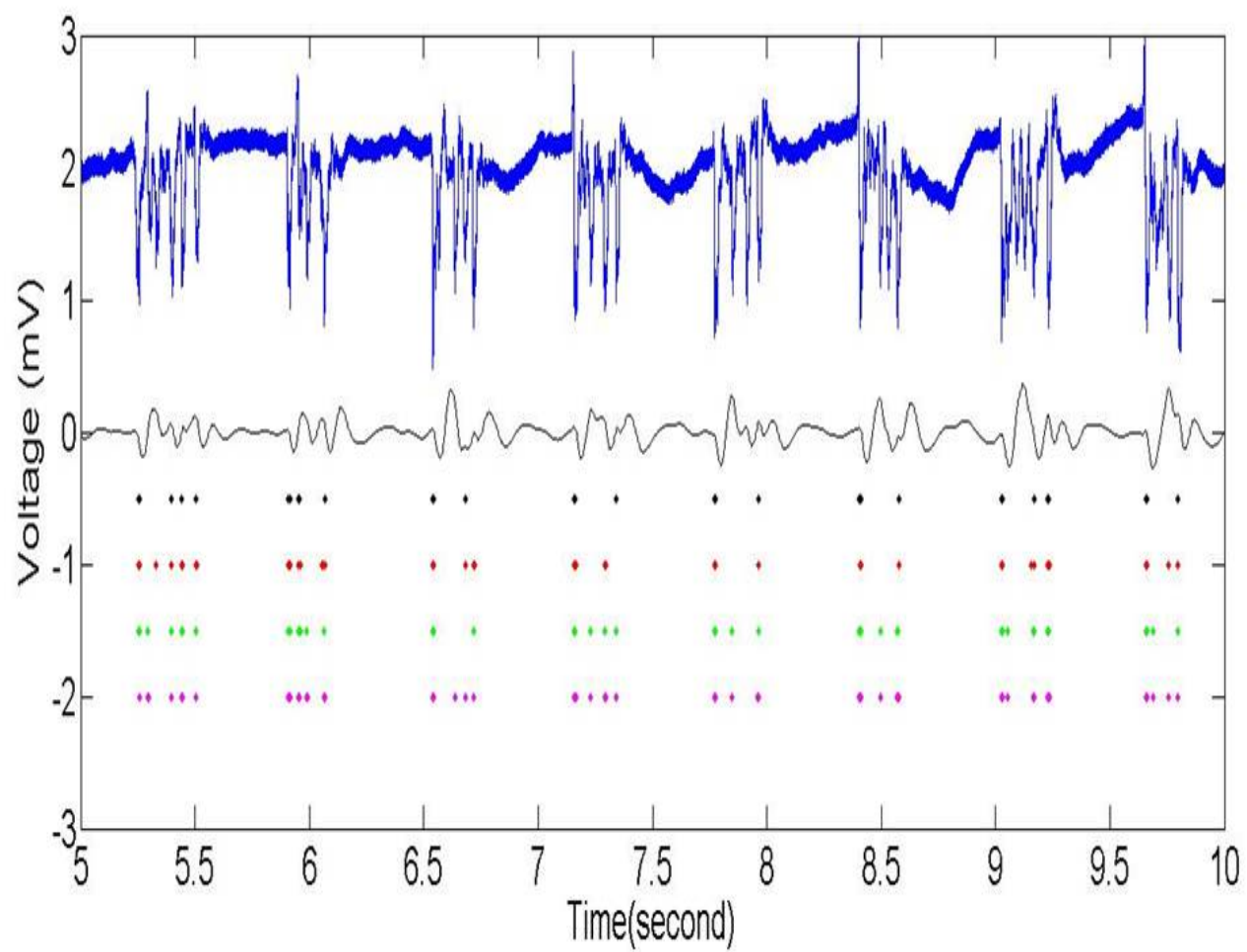

Figure 4.7: The top trace is the EEG signal of an electrically evoked seizure recorded by one channel of the microelectrode array 5 seconds after stimulation. The second waveform is the theta rhythm, which is the EEG signal band-pass filtered between 4 and $10 \mathrm{~Hz}$. The bottom dots are the raster of peaks of the firings. Each vertical tick mark represents a peak of a spike, and each row of tick marks represents the peaks of firing events in one channel over 5 seconds.

theta cycle was defined as the time between two adjacent negative peaks in the theta oscillation. Theta phase linearly increases from 0 to $2 \pi$, from one negative peak of the theta oscillation to the next. Each mark in the figure was assigned with a phase value between 0 and $2 \pi$. During an evoked seizure, theta phase distribution spread between 0 and $2 \pi$ for each channel (See Figure 4.8). The high frequency firing had a preferential relationship with the theta oscillations, with firings clustering around $1.8 \pi$. This indicates the hippocampal CA1 neurons tend to fire around the troughs of the theta oscillations. 

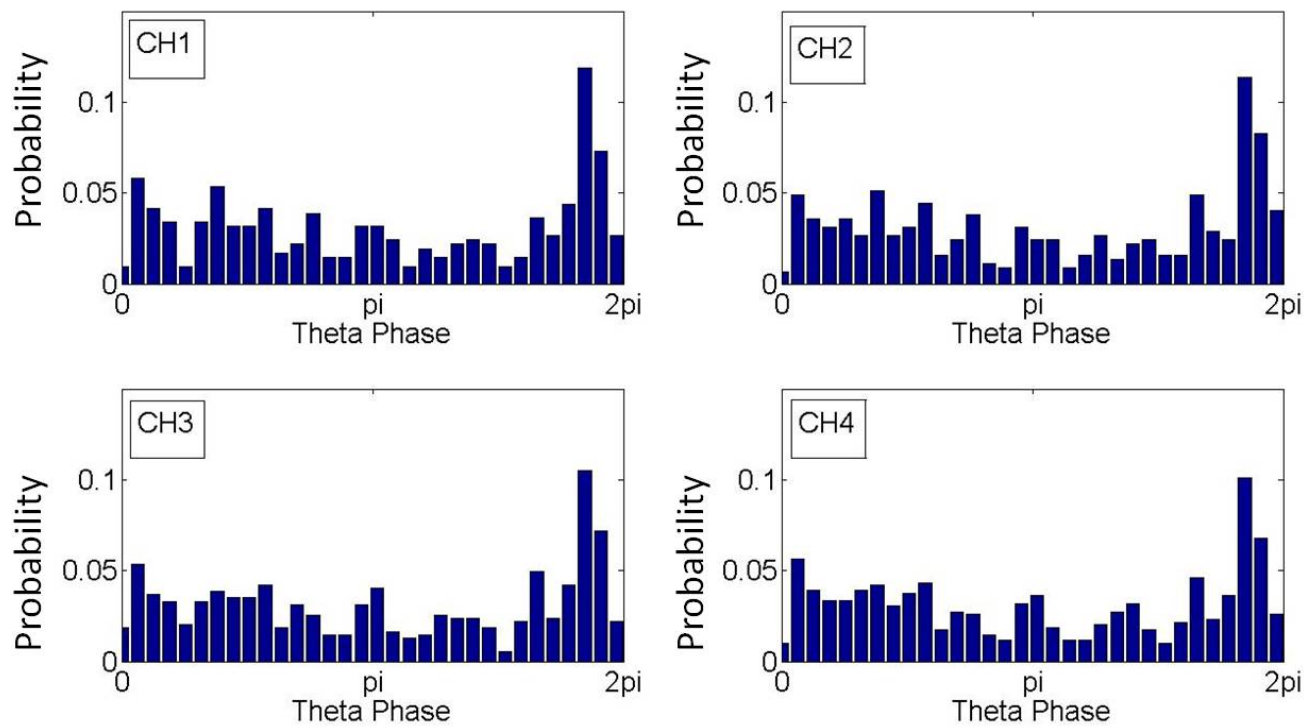

Figure 4.8: Theta phase distributions during an evoked seizure. For each channel of the microelectrode array, the theta phase distribution is spread between 0 and $2 \pi$ around a preferred theta phase. The theta phase distribution did not vary from channel to channel.

In order to understand the propagation pattern along the lamellar axis, phase differences between channels were investigated. Figure 4.9 shows the phase difference distributions between different channels. Firstly, the values of the phase differences are very small. Secondly, all the phase difference distributions were negatively skewed, which suggests that the firing recorded by one channel leads the other. Peaks of the firings in channel 1 were always earlier than in the other channels. By calculating the phase difference for all pairs, the firing pattern was shown to propagate in a linear fashion, channel $1 \rightarrow$ channel $2 \rightarrow$ channel $3 \rightarrow$ channel 4 (Figure 4.10). The major source of input to the hippocampus is the hippocampus itself. The hippocampal CA3 is connected to CA1 by Schaffer collateral connections (See Figure 2.1). Each CA3 pyramidal cell gives rise to highly collateralized axons that follow both the transverse and oblique orientations of the CA1. This firing pattern indicates the seizures were evoked by stimulating the hippocampal CA3 

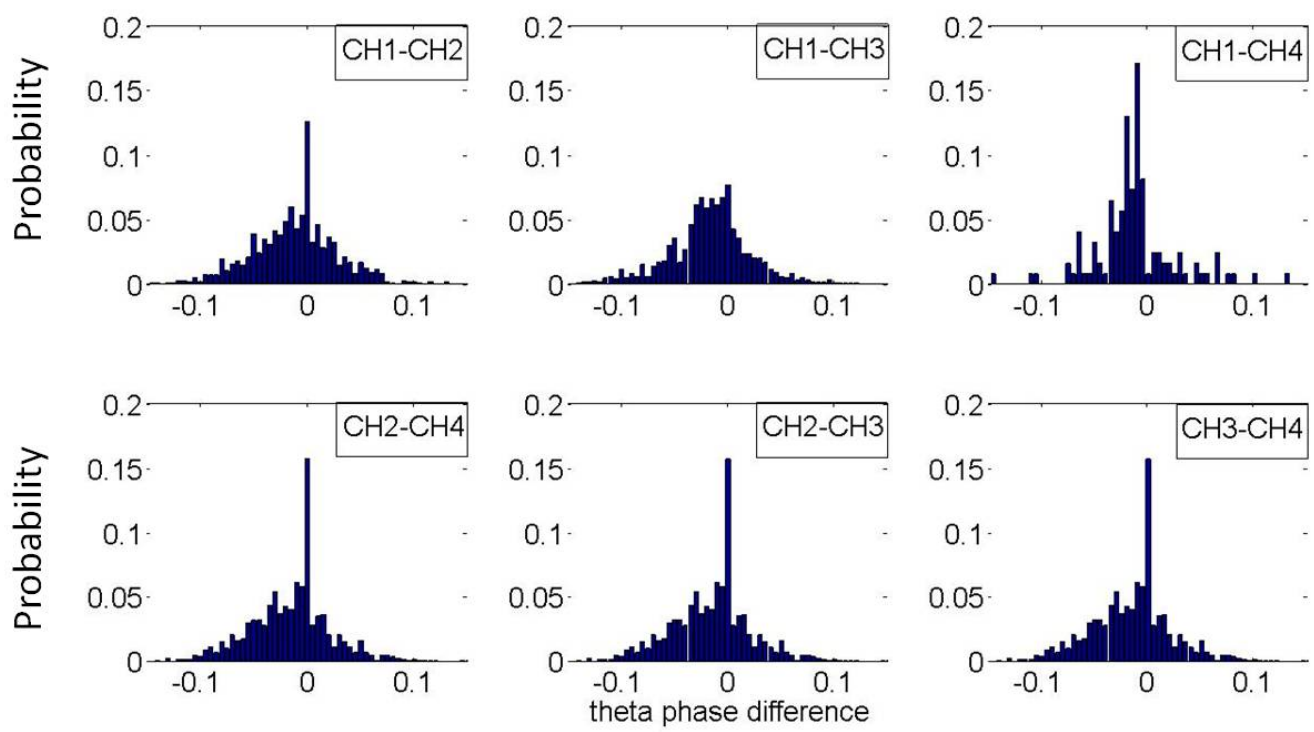

Figure 4.9: The distributions of the phase difference between different channels. Each histogram is the distribution of the theta phase difference between two channels. All the distributions are skewed with a negative mean and median, indicating that the high frequency firing recorded by one channel proceeds the other.

and they propagate along the CA1 lamellar layer- starting from the part close to CA3 and spreading out from there. The neuronal firings in CA1 were shown to be synchronous, with a propagation pattern along lamellar direction from proximal to distal in CA1 region.

\subsubsection{Event Synchronization}

The event synchronization was evaluated between all channels. A colormap of the average event synchronization level was shown in Figure 4.11. The average Q for each pair of channels is $0.77 \pm 0.03$ (channel 1-2), $0.67 \pm 0.02$ (channel 1-3), 0.70 \pm 0.02 (channel 1-4), $0.69 \pm 0.02$ (channel 2-3), $0.73 \pm 0.02$ (channel 2-4) and $0.81 \pm$ 0.03 (channel 3-4). Synchronization was the highest between channel 1 and channel 2, channel 3 and channel 4 . Other combinations did not show significant differences 


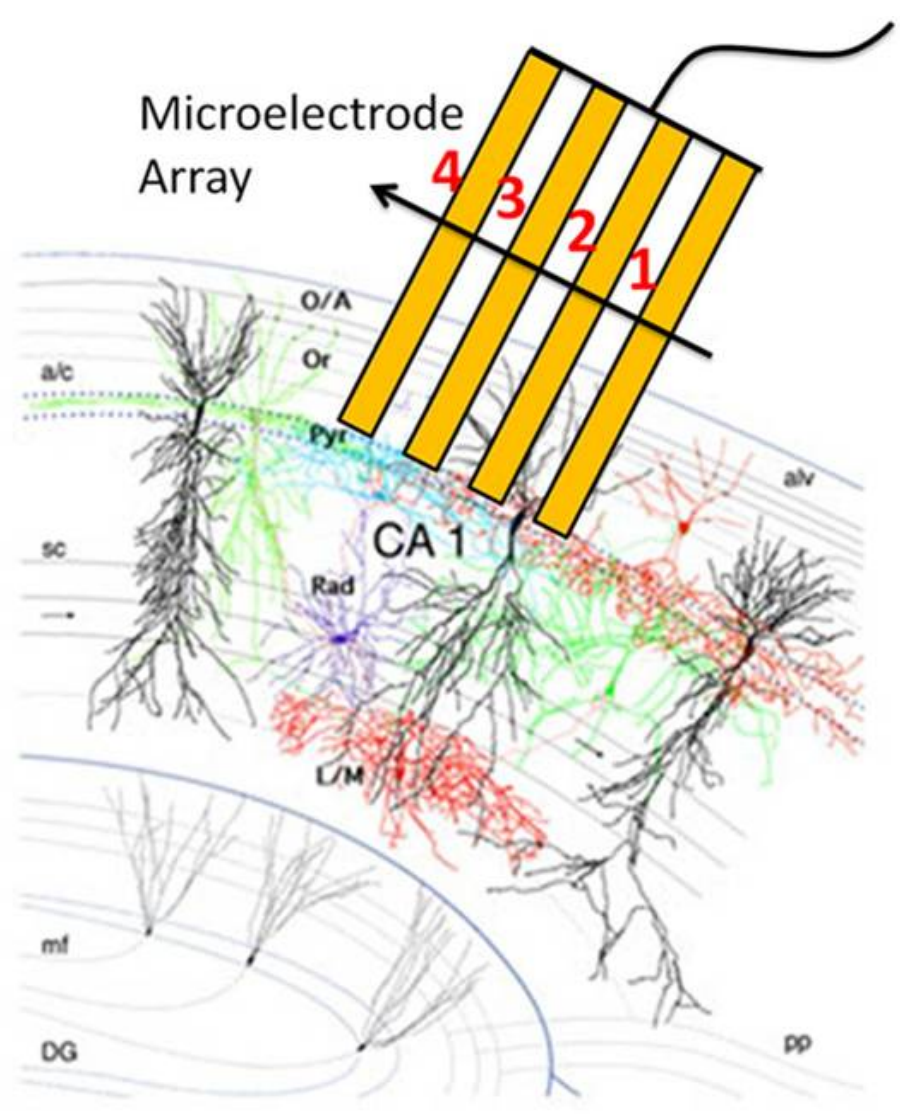

Figure 4.10: Schematic plot of the firing propagation pattern in hippocampal CA1. The arrow indicates the direction of propagation, from channel 1 to channel 4 along the lamellar axis.

between each other (ANOVA). The event synchronization suggests that the degree of neuronal firing synchrony in the small recording region is not homogeneous in the lamellar direction.

\subsection{Conclusion}

The microelectrode array has shown that it has the ability to simultaneously record from different groups of neurons. The underlying assumption is that each electrode recorded from one small local group of neurons. The electrodes are 


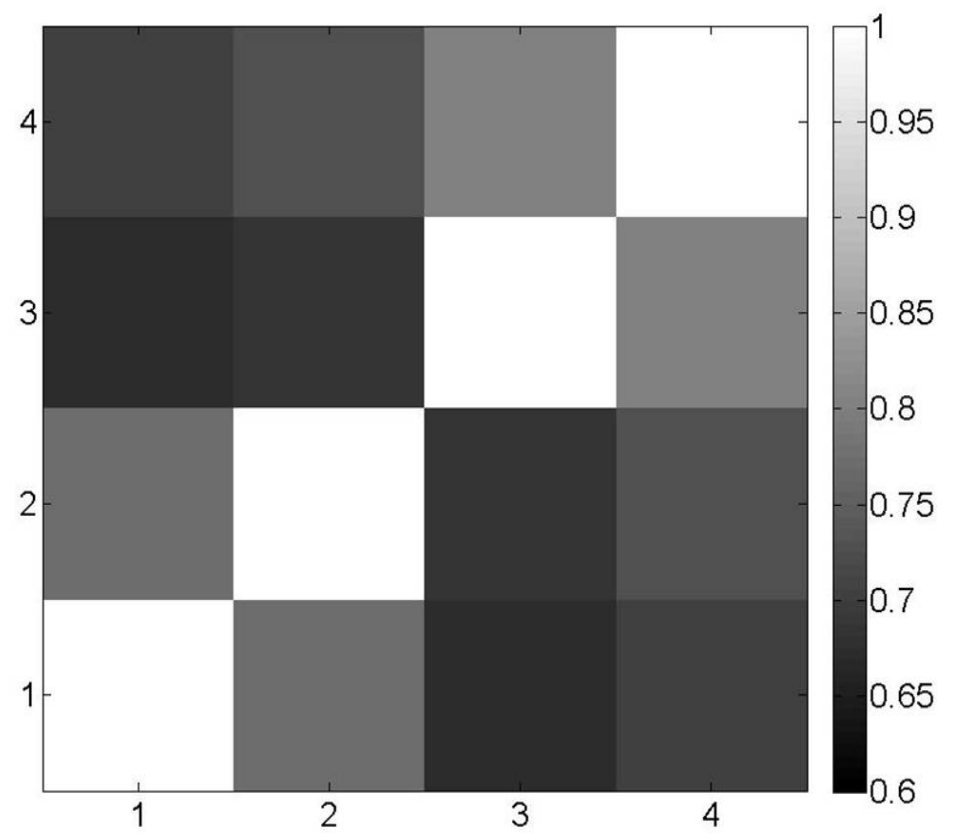

Figure 4.11: An example of the event synchronization level of high frequency neuronal firing. This is a $4 \times 4$ event synchronization strength matrix. The columns and rows are indexed by the channel number 1-4 of the microelectrode array.

spaced $50 \mu \mathrm{m}$ from each other, which is the diameter of a pyramidal cell soma. The firing figure showed that some neurons are recorded by two or more channels in the recording. Because of this, it cannot be said that the recording is a single unit recording. However, the goal of this experiment was not to count how many neurons were represented by each electrode. What interested us was how high frequency firing interacts and synchronizes in a small local region in the hippocampus CA1 cell layer. So, knowing that each electrode represents a small group of neurons was sufficient for our analysis.

The kindling protocol successfully generated recurrent seizures in anesthetized rats. The ADD of the evoked seizures increased with the number of stimulus exposures for each rat. The average ADD varied substantially from animal to 
animal.

We also studied neuronal synchronization during electrically evoked seizures in anesthetized rats. Three different synchronization measurements were applied to examine different aspects of high frequency neuronal firing. (1) The cross correlation showed that the firings was maximally correlated in the middle phase of a seizure. Both the beginning and the end stage of a seizure were less correlated. (2) Theta phase synchronization quantifies the synchrony level of each channel. Shannon entropy is an indicator of the synchrony of the theta phase distribution. All channels share a very similar theta phase distribution with a preferred firing phase during kindled seizures. The phase distributions indicated that hippocampal CA1 neurons tended to fire around the negative peaks of the theta oscillations. Similar results were reported by Holmes et al. [9], who found that the hippocampal pyramidal cells fired preferentially on the peaks of the CA1 theta oscillation. The theta phase difference distribution reveals the propagation pattern in hippocampal CA1 cell layer. We tested the lamellar hypothesis in this anesthetized animal experimental setup and found that high frequency firing of hippocampal neurons in the CA1 cell layer propagated along the lamellar axis and activated in a stripe-like fashion. The propagation direction is from proximal to distal in CA1 region. (3) Event synchronization determines the synchrony levels between channels in a small recording area. It demonstrates that synchrony is a function of distance between recording sites. Therefore the synchrony level was higher between the two closest recording sites, and lower between the others.

Overall we proved that in the anesthetized rat, electrically evoked seizures were not hypersynchronized along the lamellar axis because the synchronization level varied temporally and spatially within a small local area. However, there are several limitations to this experimental setup. Because the rats were under 
CHAPTER 4. SYNCHRONIZATION ALONG THE CA1 LAMELLAR AXIS IN

anesthesia, it is difficult to quantify the severity of the seizures through commonly used methods, with the exception of the ADD. Additionally, both normal EEG baseline and seizure activity were suppressed by the anesthetic drug. In order to overcome these problems, the kindling protocol was altered for awake animals in Chapter 5 . 


\section{Chapter 5}

\section{Synchronization along the CA1}

\section{Lamellar and Septotemporal Axes in}

\section{Awake Rats}

The purpose of this study was to understand the structure of synchronization and propagation in the hippocampal CA1 cell layer during electrical kindling in awake rats. Multiple simultaneous extracellular recordings were performed along the lamellar and septotemporal axes in the hippocampal CA1 cell layer. The dynamic synchronization and propagation of neuronal firings along both axes during evoked seizures were analyzed by using theta phase synchronization and event synchronization. More details on these two methods are in section 3.9.

\subsection{Evoked Seizures}

We were interested in the temporal relationship between synchrony and seizure severity. We were interested in determining how synchrony varies across a single 
seizure, and how synchrony changes during the kindling. Compared to chemoconvulsive methods, the kindling protocol was designed to electrically evoke seizures periodically over a relatively long period of time in order to evoke epileptogenesis. This method allows synchrony changes as kindling progresses to be investigated.

We delivered 10 minute long electrical pulse train stimulus to the hippocampal CA3 region every half an hour. The evoked seizures were detected and recorded immediately after the stimulus. After the first stimulus, an electroencephalographic seizure was evoked and high amplitude rhythmic seizure activity was clearly visible on all channels. Low-noise and artifact-free EEG recordings were selected for future analysis. The morphology of the firings changed throughout the seizure. Particular features of this EEG recording were enlarged to show temporal details (Figure 5.1). The amplitude of the firing events attenuated at the end phase of the evoked seizure.

The electrodes in the microelectrode array were spaced by the placement of a 50 $\mu \mathrm{m}$ wire between each electrode, which is about the diameter of a pyramidal cell body. There was also a thin layer of superglue applied to each electrode in order to fix the electrode wires in place. The spike waveform changes between channels during the evoked seizures showed that it is possible for the electrodes to record from different groups of neurons in the densely packed CA1 pyramidal cell layer. Our interpretation of our data is based on the assumption that each channel of the microelectrode array recorded from a largely unique set of neurons, with minimal crosstalk. 

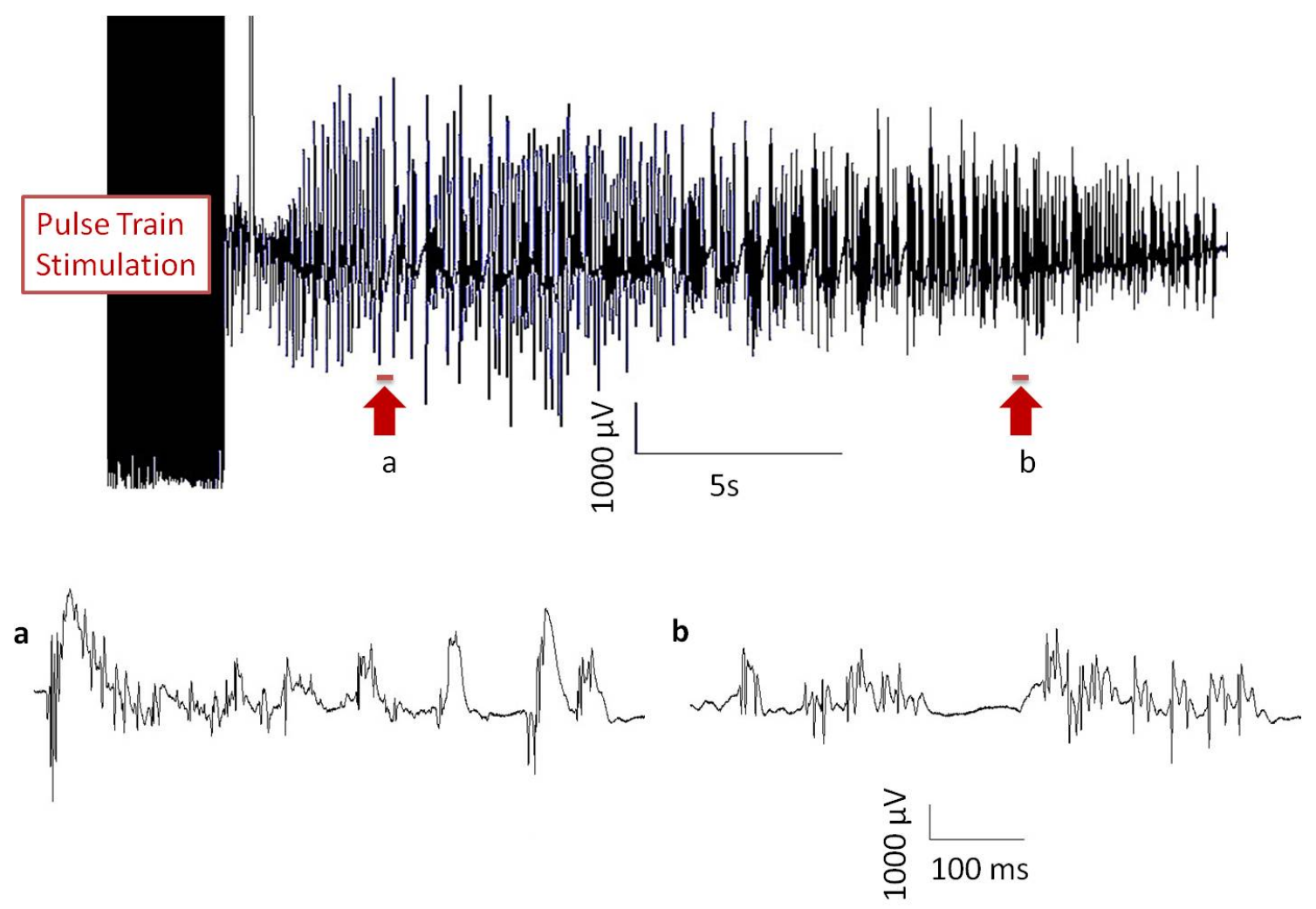

Figure 5.1: (Top) An example of an electrically evoked seizure was recorded in an awake rat immediately after 10 seconds of stimulation. The EEG was filtered above $1 \mathrm{~Hz}$. (Bottom a and b) Expanded views of two segments of the EEG data, taken from the sections indicated by the arrows beneath the top trace.

\subsection{Behavioral Seizure Scores and After-discharge Du- ration}

In order to quantify the severity of the evoked seizures, two commonly accepted measurements were used. One method was human categorization of the behavioral seizure stage (in this case Behavioral Seizure Score). During the seizures, behaviors were scored based on the Racine five-point method (see section 3.6). The other measurement used was after-discharge duration.

Figure 5.2 showed an example of ADD and BSS progression across a four-day 


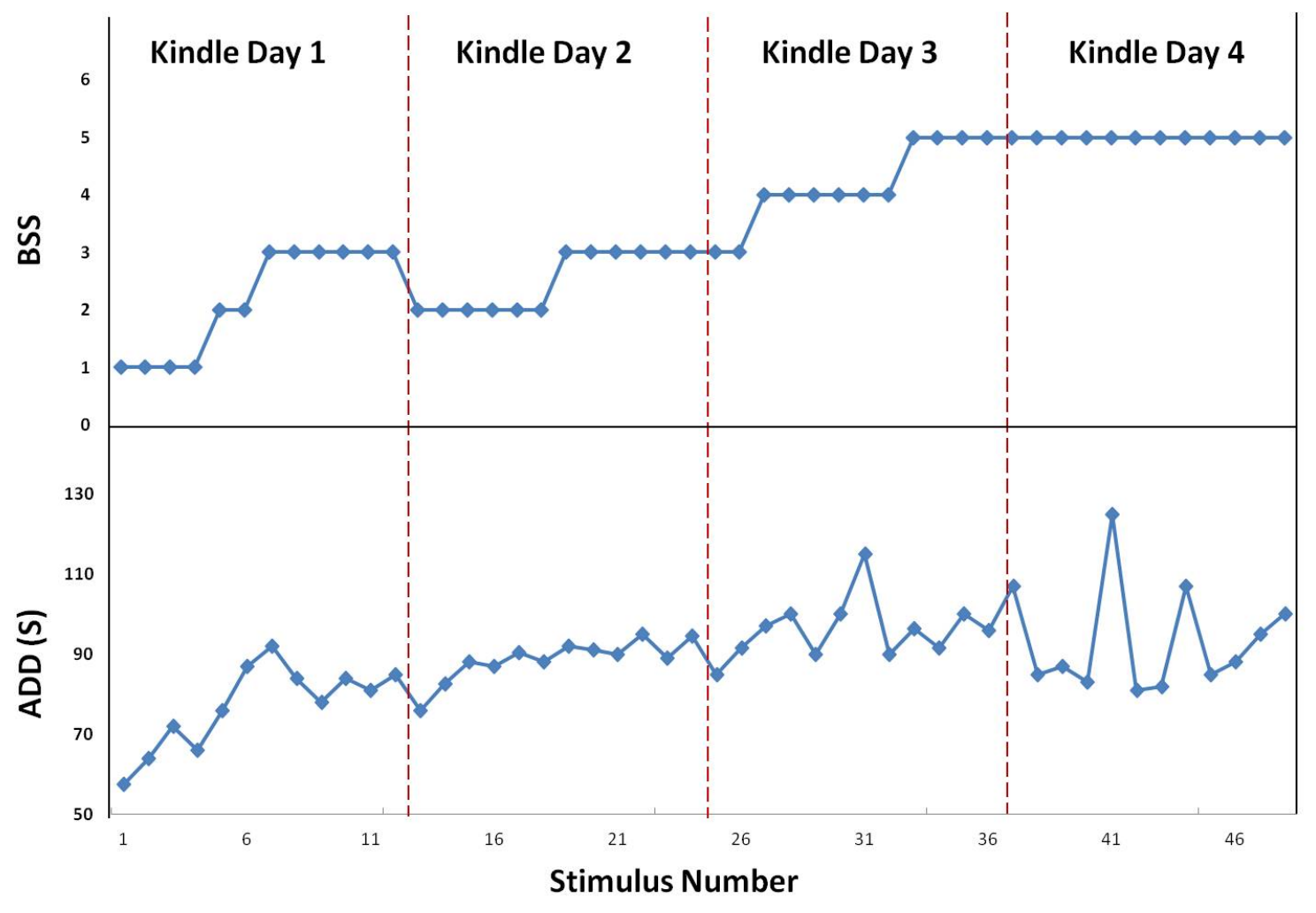

Figure 5.2: Behavioral seizure scores and after-charge duration increased in severity during kindling.

kindling for one animal. Animals were kindled until they reached BSS 5 . The amount of time it took to accomplish this varied from rat to rat, averaging around 2-5 days (approximately 24-60 sets of electrical stimuli). However, behavioral seizure stages demonstrated considerable volatility- sometimes relapsing to prior stages and at other times temporarily leaping ahead. The kindling protocol was not $100 \%$ effective either, with 3 out of 23 rats failing to reach or maintain stage 5.

ADD increased rapidly on the first day of kindling, increasing from 57.5 to 92 seconds (60\% increase). On days 2 and 3, the ADD did not substantially change, averaging $92.3 \pm 7.4$ seconds. On day 4 , when this rat was fully kindled, the mean ADD was $93.8 \pm 13.6$ seconds. The relatively large variation was due to the fact that after a long seizure, neurons need time to recover- hence longer seizures were 
typically followed by comparatively shorter ones.

\subsection{High Frequency Firing}

The signal from the microelectrode array was filtered between 300 and $4000 \mathrm{~Hz}$ prior to synchronization analysis. In figure 5.3 and figure 5.4, segments taken

from microelectrode arrays in both the lamellar and septotemporal configurations are shown. The neuronal firings taken from seizures that were recorded in both the lamellar and septotemporal configurations were organized topographically. The different recordings sites, which correspond to different electrodes of the microelectrode array, varied accordingly in their response latencies to the stimulation. In the lamellar configuration, seizures showed a growing preference for high frequency activities as the seizure progressed. Stage 5 seizures had substantially more high frequency activities than the lower stage seizures. We observed high frequency firing events throughout the seizure. While in the septotemporal direction, the firing frequency of stage 5 seizures seemed to remain the same as that of stage 1 seizures. The firing frequency during seizures was quantified in next section.

\subsection{Spike Rate}

A peak finder (run by MATLAB) was applied to the filtered data to find all of the peaks and troughs of the spikes. The code is attached at the end of this dissertation. The peak finder was coded to find the local maximums and minimums. Spike rate (the number of spike events in one second) was calculated during the evoked seizures.

Figure 5.5 shows the spike rate distributions of stage 1 and 5 seizures in both the 
A

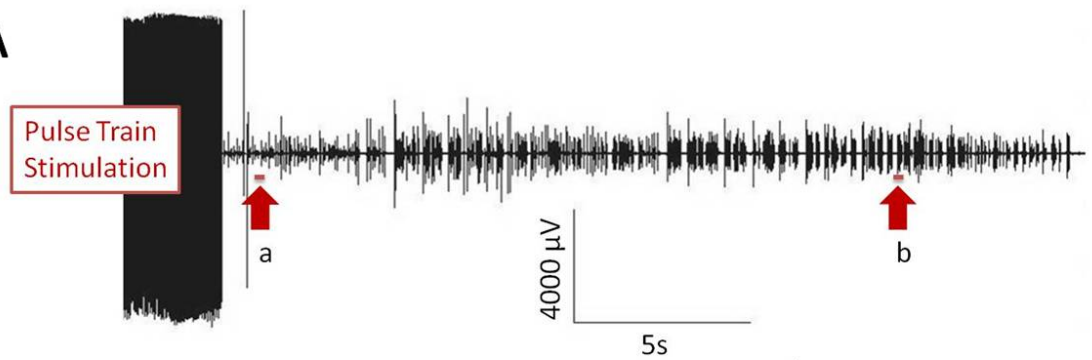

a
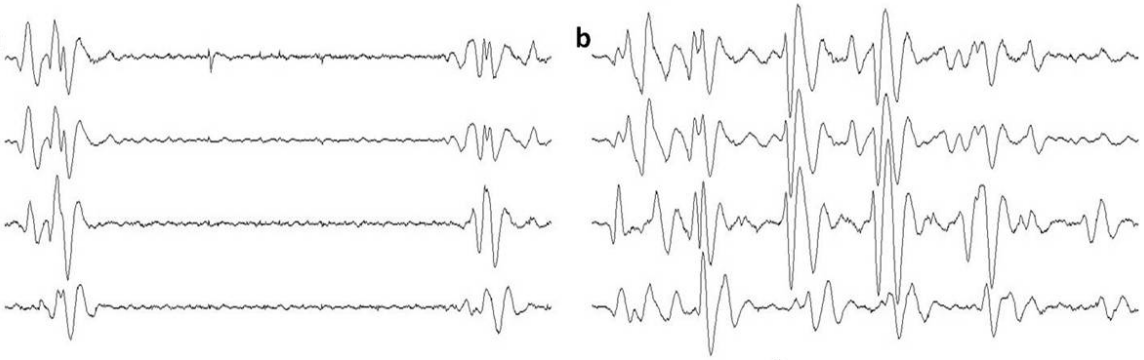

B

$$
\text { 인 난 }
$$

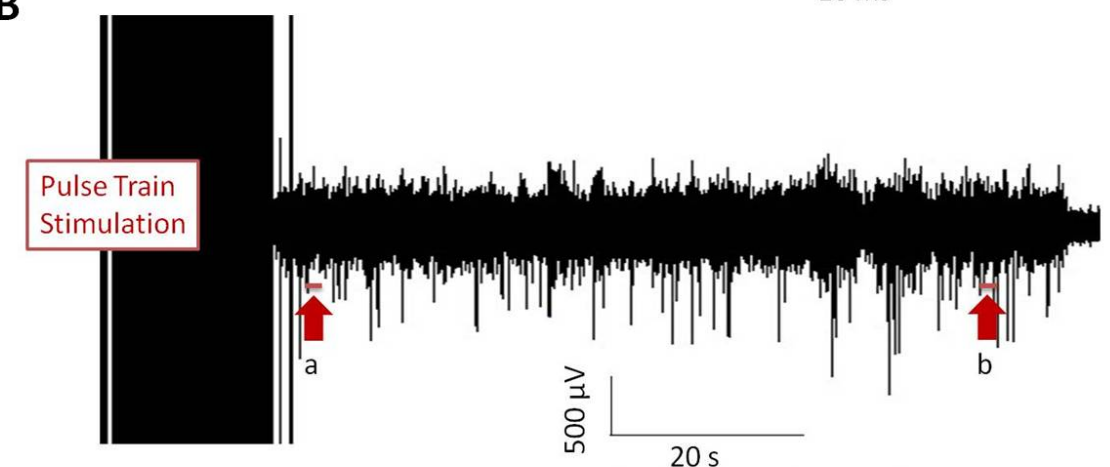

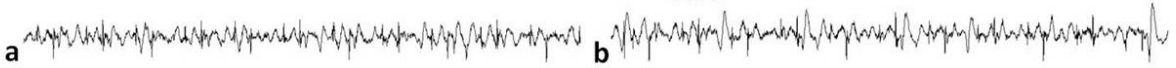

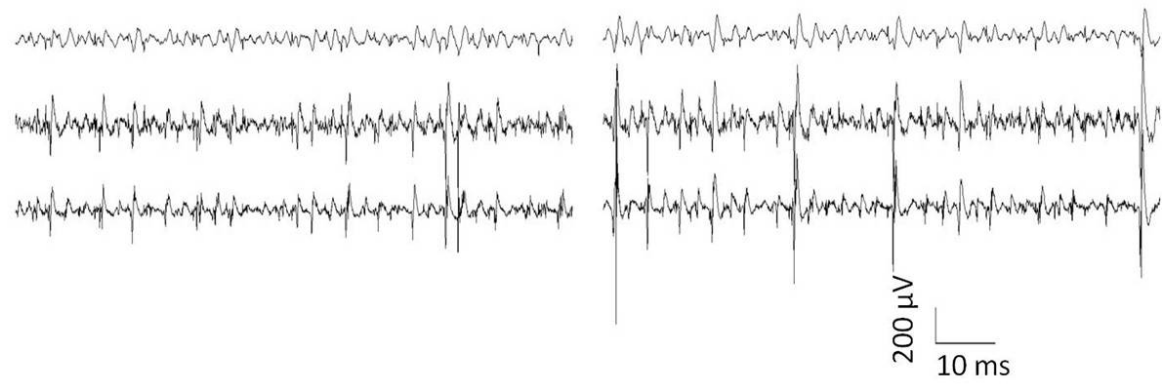

Figure 5.3: High frequency firing along the lamellar axis. EEG was filtered between 300 to $4000 \mathrm{~Hz}$ to extract high frequency firing data. High frequency firing during a stage 1 seizure (A) and a stage 5 seizure (B). The top traces are one channel of the filtered seizure data. Bottom a and $b$ traces are expanded views of the top traces indicated by the arrows. The schematic figure of the microelectrode array placement along the lamellar axis is on the right. 
A

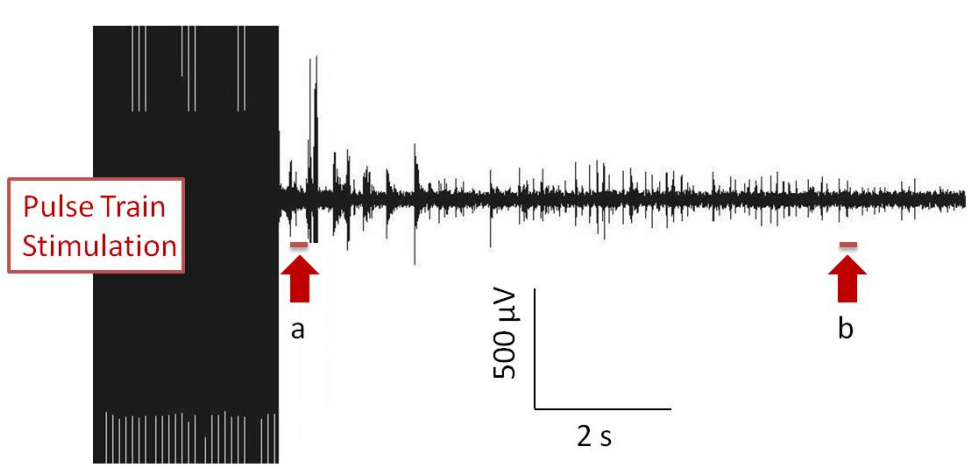

a

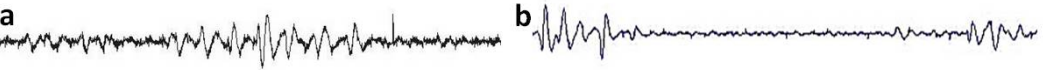

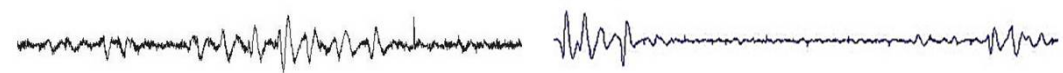
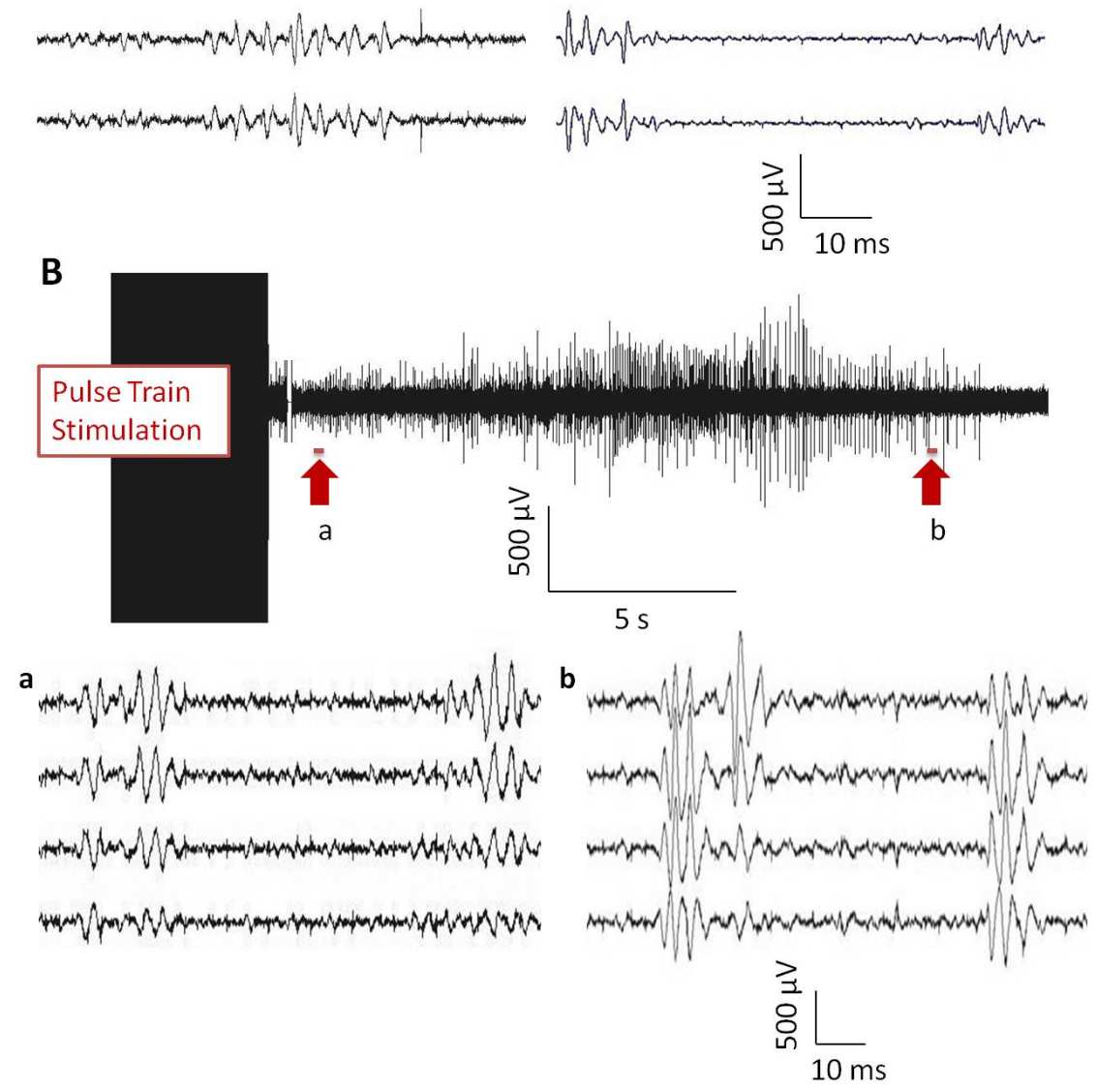

Figure 5.4: High frequency firing along the septotemporal axis. High frequency firing during a stage 1 seizure (A) and a stage 5 seizure (B). The top traces are one channel of the filtered seizure data. Bottom a and b traces are expanded views of the top traces indicated by the arrows. The schematic figure of the microelectrode array placement along the septotemporal axis is on the right. 

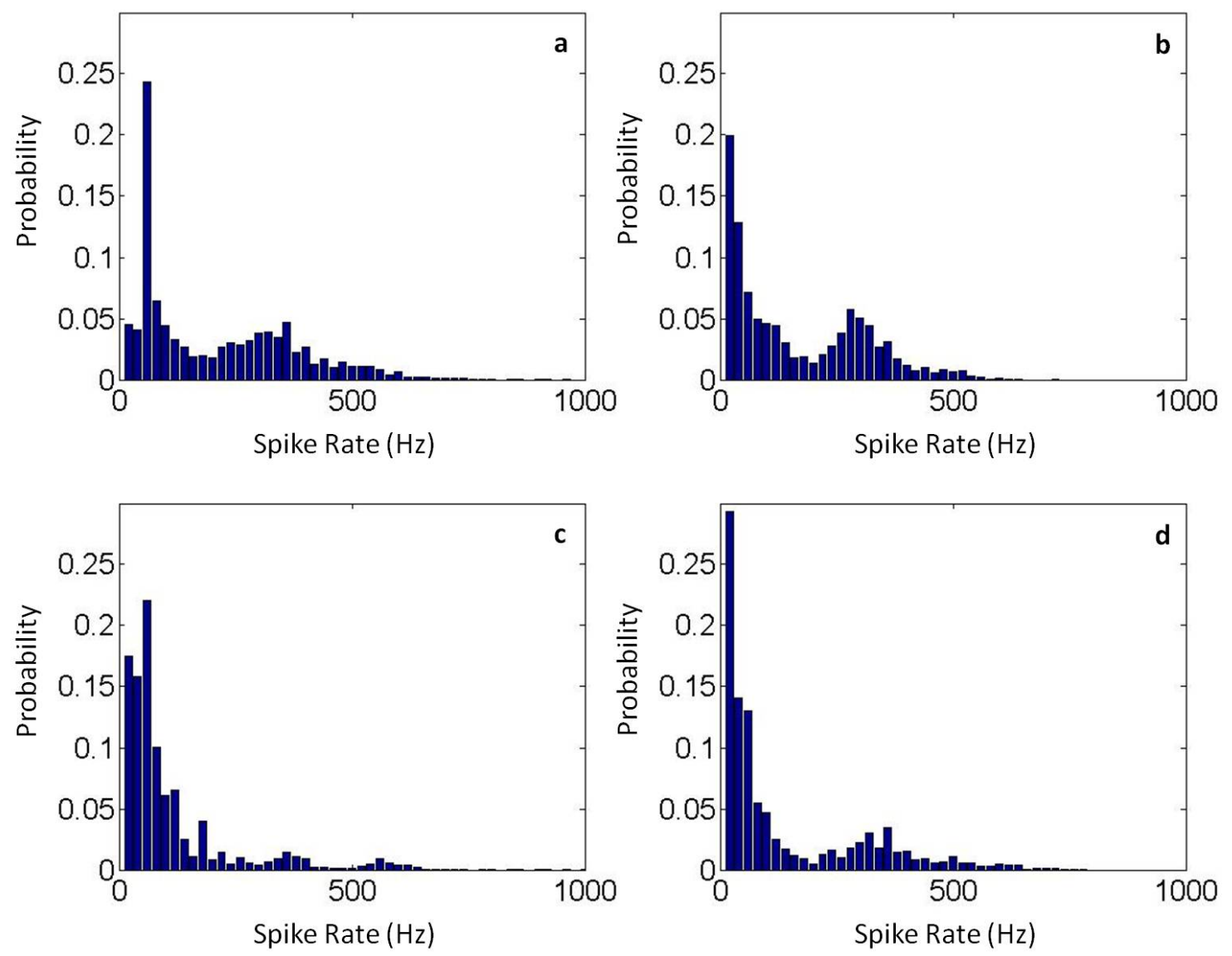

Figure 5.5: Spike rate distributions during kindling. Spike rates of stage 1 and stage 5 seizures in the lamellar configuration $(a, b)$ and the septotemporal configuration $(c, d)$.

lamellar and septotemporal configurations. The high frequency component (above $300 \mathrm{~Hz}$ ) increased as the seizure stage advanced in both the lamellar and septotemporal configurations. For the lamellar configuration, the spike rate distribution of the stage 1 seizures was significantly different from that of the stage 5 seizures (p $<0.05$, Kolmogorov-Smirnov test). Conversely, in the septotemporal configuration, there was no significant difference between the spike rates of the stage 1 and the stage 5 seizures $(\mathrm{p}=1$, Kolmogorov-Smirnov test). 

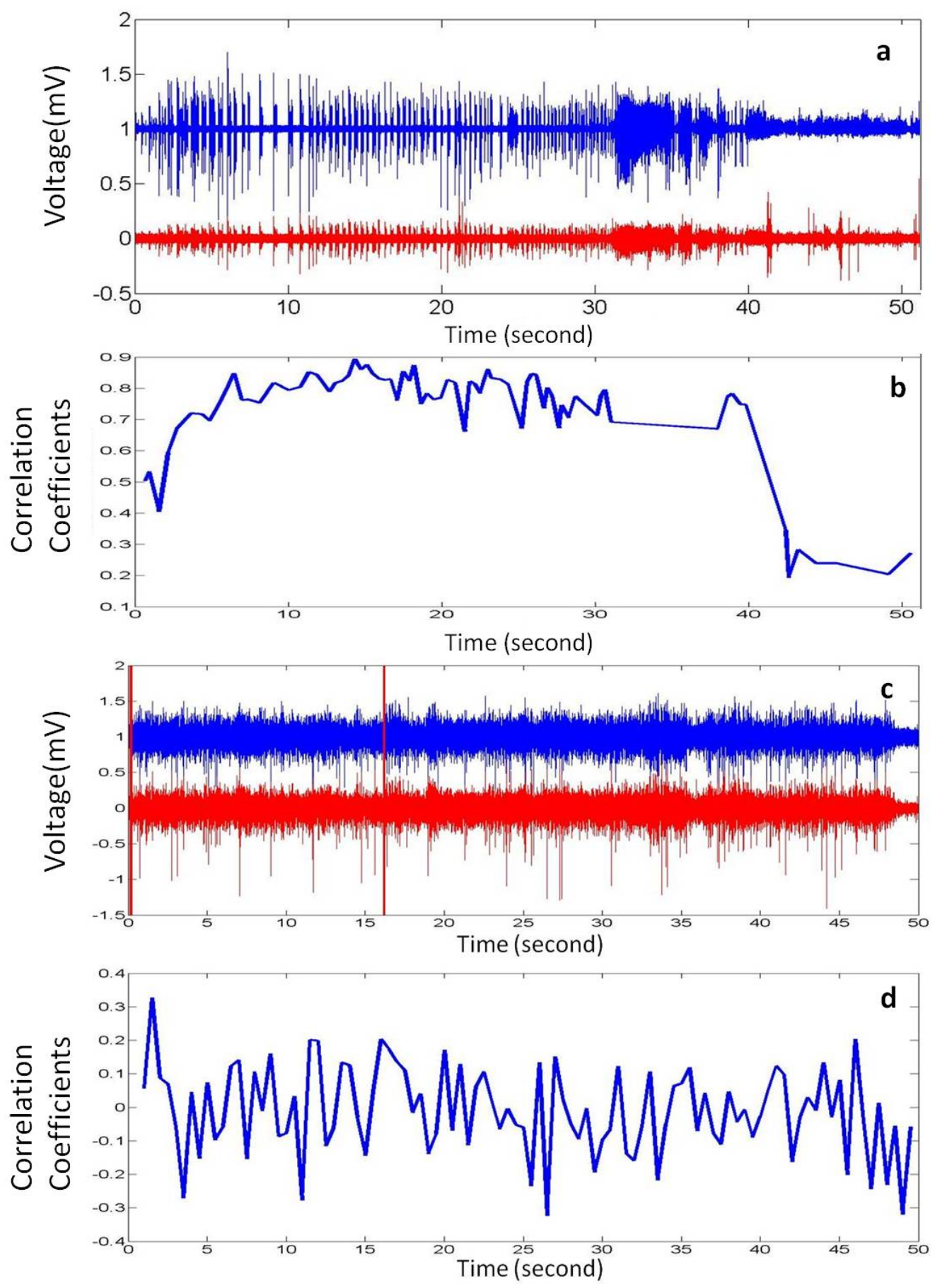

Figure 5.6: An example of the correlation profile between two channels of the microelectrode array recording during seizures in awake rats. EEG data and correlation coefficients are shown in (a and b) lamellar configuration and (c and $d$ ) septotemporal configuration. 


\subsection{Cross Correlation}

Cross correlation coefficients were calculated between each channel, as described in chapter 4 . For a visual example of cross correlation, Figure 5.6(a) for the sake of comparison, plotted two time series next to each other. And Figure $5.6 \mathrm{~b}$ graphed the correlation coefficient against time. During an electrically evoked seizure, the correlation coefficients rapidly increased from 0.42 to 0.84 during the first 8 seconds of seizure, stayed between 0.68 and 0.9 for the middle stage and dropped to about 0.21 at the end of this seizure. Correlation was disrupted at the end of the electrically evoked seizure, much like what was shown in anesthetized rats in last chapter. This may suggest that correlation is an important factor in gauging both the development of a seizure, and its natural inhibition.

In the septotemporal configuration, the correlation coefficients fluctuated randomly between -0.29 to 0.33 throughout the duration of the seizure, as shown in Figure $5.6 \mathrm{c}$ and $\mathrm{d}$. The negative correlation coefficients indicated that when the voltage of one channel increased, the voltage of the other channel decreased. Since these coefficients were quite small when compared to those of the lamellar configuration, it is not surprising that that there was little-to-no correlation in the septotemporal direction.

\subsection{Theta Phase Synchronization}

\subsubsection{Oriented Along the Lamellar Axis}

We recorded 150 evoked seizures from 6 animals with the microelectrode array placed along the lamellar axis. Theta phase analysis of these seizures robustly demonstrated that neuronal synchrony increased in the lamellar direction as kin- 


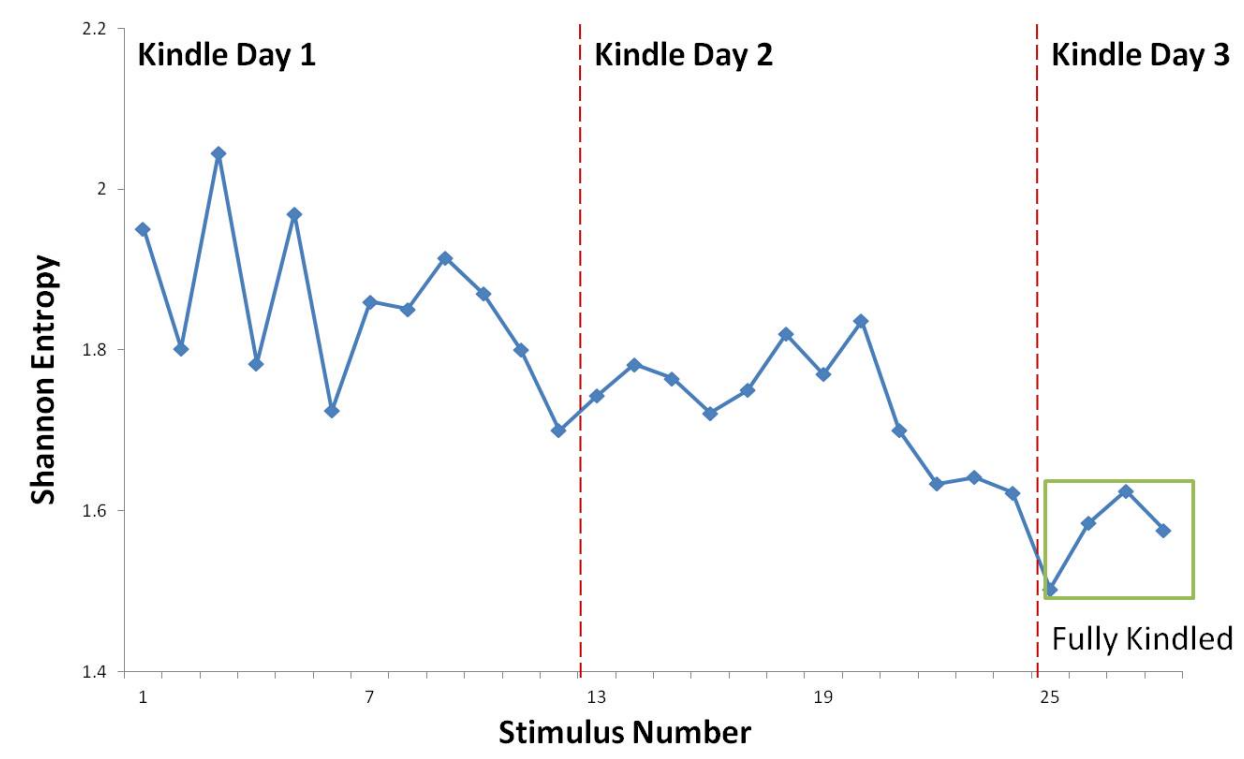

Figure 5.7: Shannon entropy attenuated as kindling progresses in the lamellar configuration.

dling progressed. Shannon entropy was calculated to quantify the degree of order in the theta phase distributions.

Temporally, figure 5.7 shows that Shannon entropy decreased significantly as kindling progressed ( $\mathrm{p}>0.05$, ANOVA). As the BSS stage advanced from 1 to 5 , the entropy level dropped for all four channels. Shannon entropy was significantly different between stage 1 and stage 5 in 3 out 4 channels (ANOVA, post-hoc)(See Figure 5.8). The mean Shannon entropy was $1.95 \pm 0.03$ for stage 1 seizures, and it decreased significantly to $1.51 \pm 0.05$ at the fully kindled stage $(\mathrm{p}<0.001$, ANOVA). The huge decrease in entropy quantitatively demonstrates that neuronal synchrony increased in the small monitored area as kindling progressed. It shows that the more severe the seizures are, the more synchronized the neurons are.

Spatially, Shannon entropy showed no significant differences between the four channels during stage 1 and stage 5 seizures ( $p>0.05$, both ANOVA and kruskalwallis test). This indicates that the level of synchrony do not vary significantly within the small recording area. 


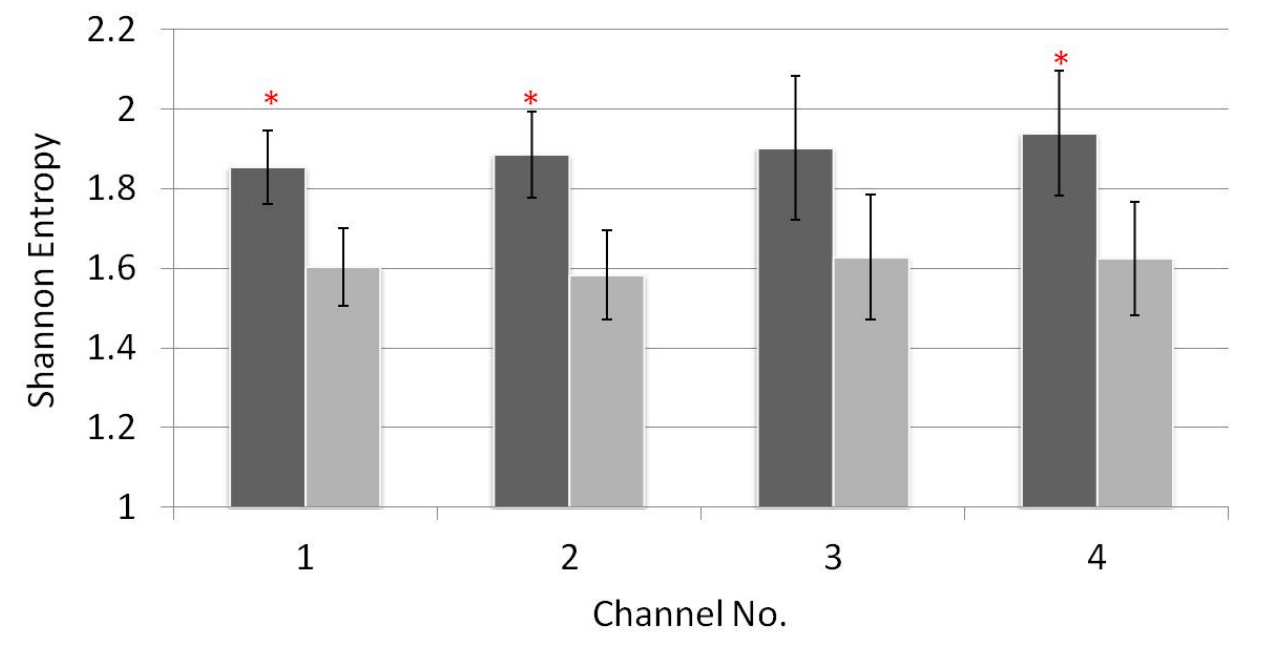

Figure 5.8: Shannon entropy of stage 1 seizures are represented by the black bar; and stage 5 seizures by the gray bar. Shannon entropy showed no significant differences between four recording channels throughout the kindling process. As the BSS stage advanced from 1 to 5 , the entropy level dropped for all four channels. Shannon entropy was significantly different between stage 1 and stage 5 in 3 out 4 channels. (ANOVA, post-hoc)

The theta phase distributions of stage 1 and stage 5 seizures are shown in Figure 5.9. They showed that during the early stage of kindling, the phase value was randomly spread between 0 to $2 \pi$, which indicates that the relationship between CA1 cell firing and theta rhythm wadfs random. This even distribution suggests that the likelihood that a given neuron will fire at arbitrary phase value $\mathrm{x}$, is the same as for any other values between 0 and $2 \pi$, which means that local neuronal firings are unaffected by the underlying theta oscillations. For stage 5 seizures, the phase distribution showed a preferred value of around $1.8 \pi$, which is close to the troughs of the theta oscillations. This is very similar to the distribution seen in anesthetized rats. The development of a preferred firing phase indicates that local neuronal firings became more strongly influence by the slow theta oscillations. Additionally, the marked transition from unaffected to strongly affected indicates that firings have become more organized as the animal was kindled. 


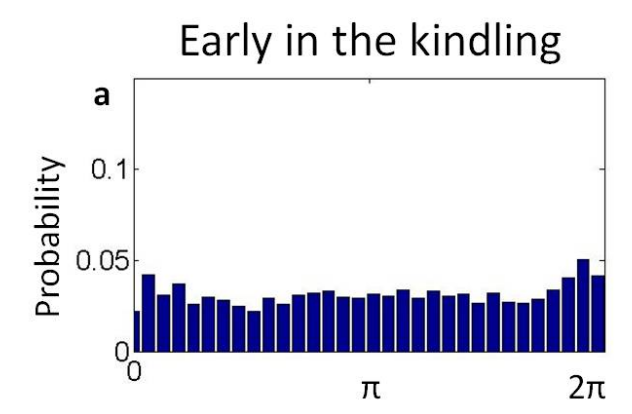

Theta Phase

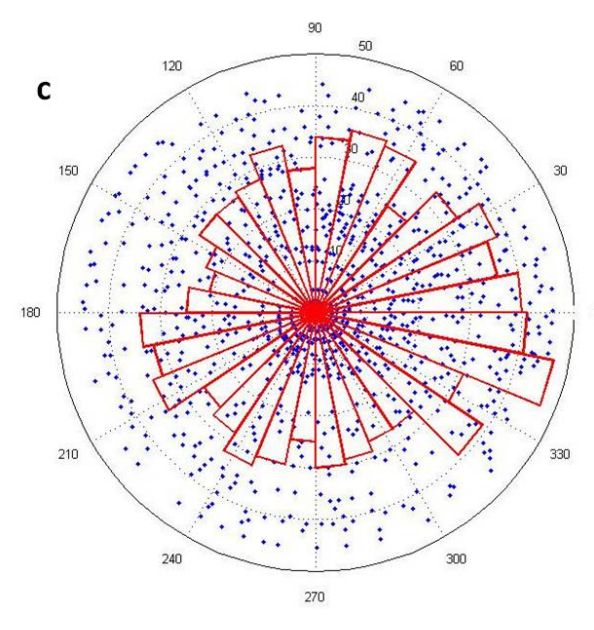

Fully Kindled

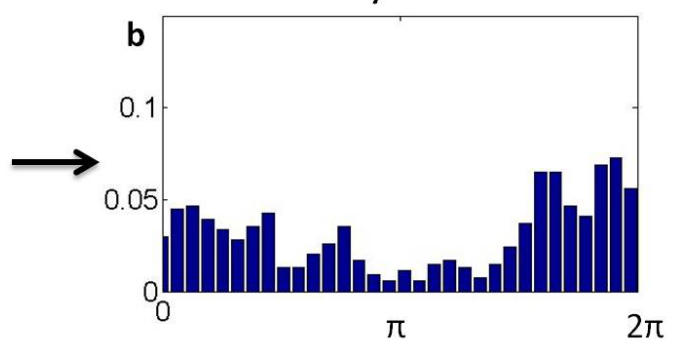

Theta Phase

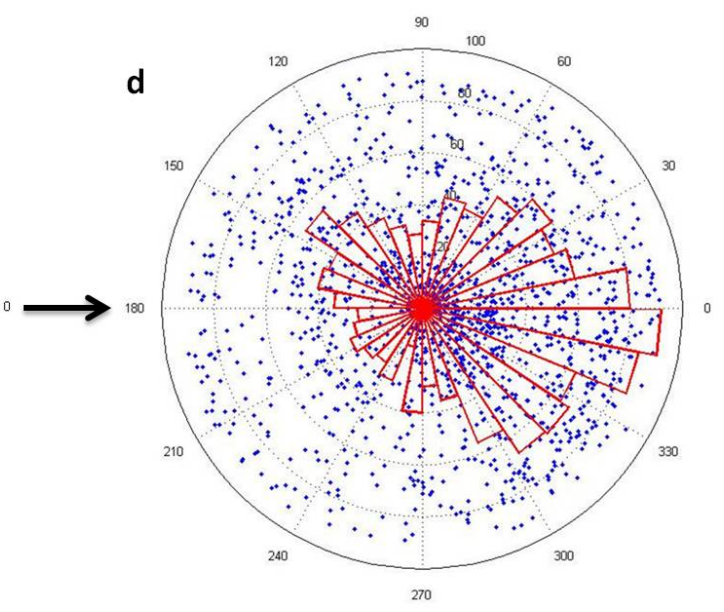

Figure 5.9: Theta phase distribution showed a preferred firing phase for stage 5 seizures. Theta phase distribution in the early stage of the kindling (a) and late stage of the kindling (b). Circle plot of the data in a and b (c and $\mathrm{d}$ respectively), graphed from 0 to $2 \pi$. All the dots represent firings during seizures.

Theta phase differences were calculated between all pairs of the channels, which in this case is 6 pairs. For the early stage seizures, all six distributions were negatively skewed (see Figure 5.10). The median and mean were negative for all distributions, which indicate that the firing recorded by one channel led the other channel. The microelectrode array channels were marked from 1 to 4 when the array was fabricated. The entire array was then implanted along the CA1 lamellar axis, and placed so that channel one was close to the hippocampal CA3 region. The neuronal firings recorded by the microelectrode array reveal propagation where 

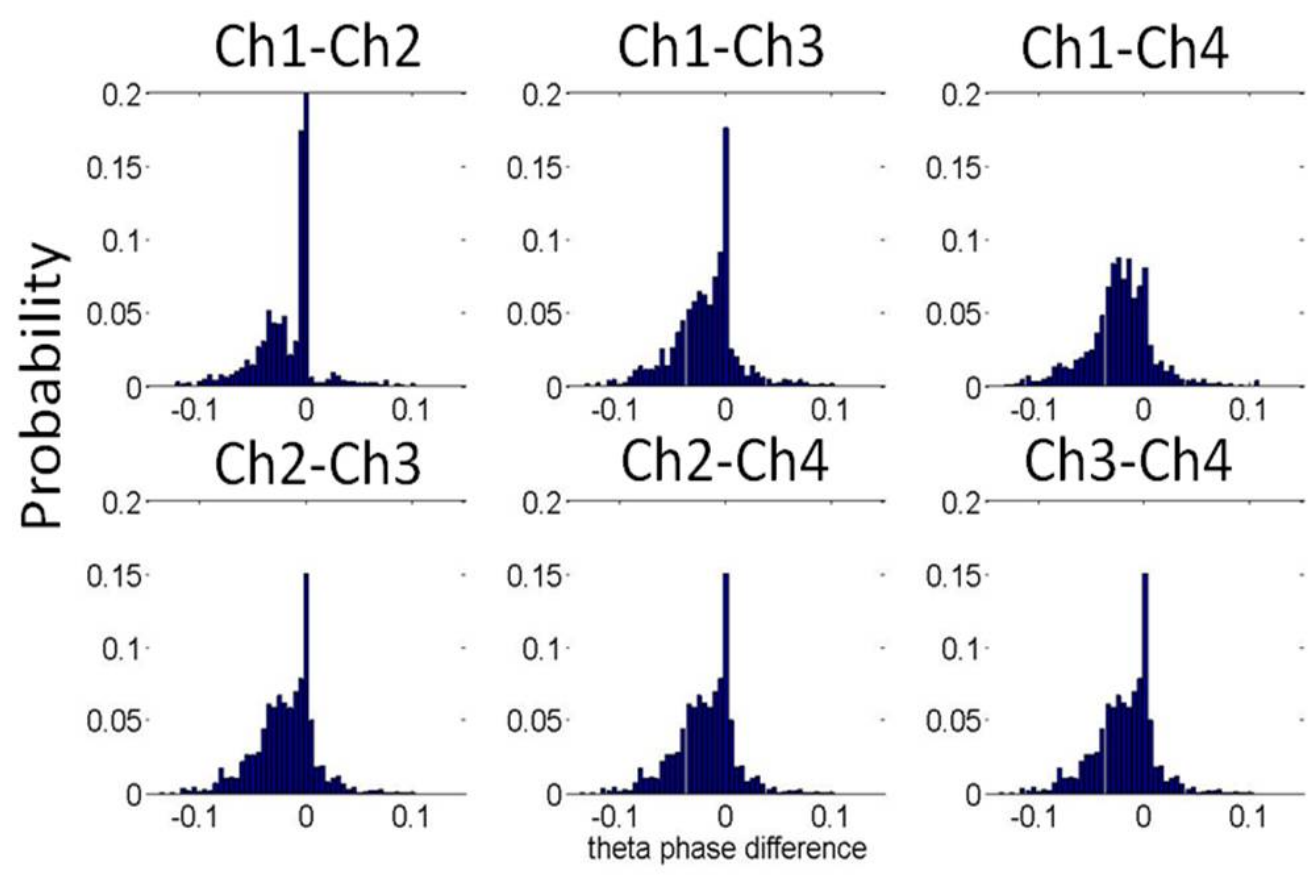

Figure 5.10: In ealry stage seizures, theta phase difference distributions among all pairs of the four channels are negatively skewed. This indicates that there is a propagation pattern between the channels of the microelectrode array that progresses in a proximal to distal manner.
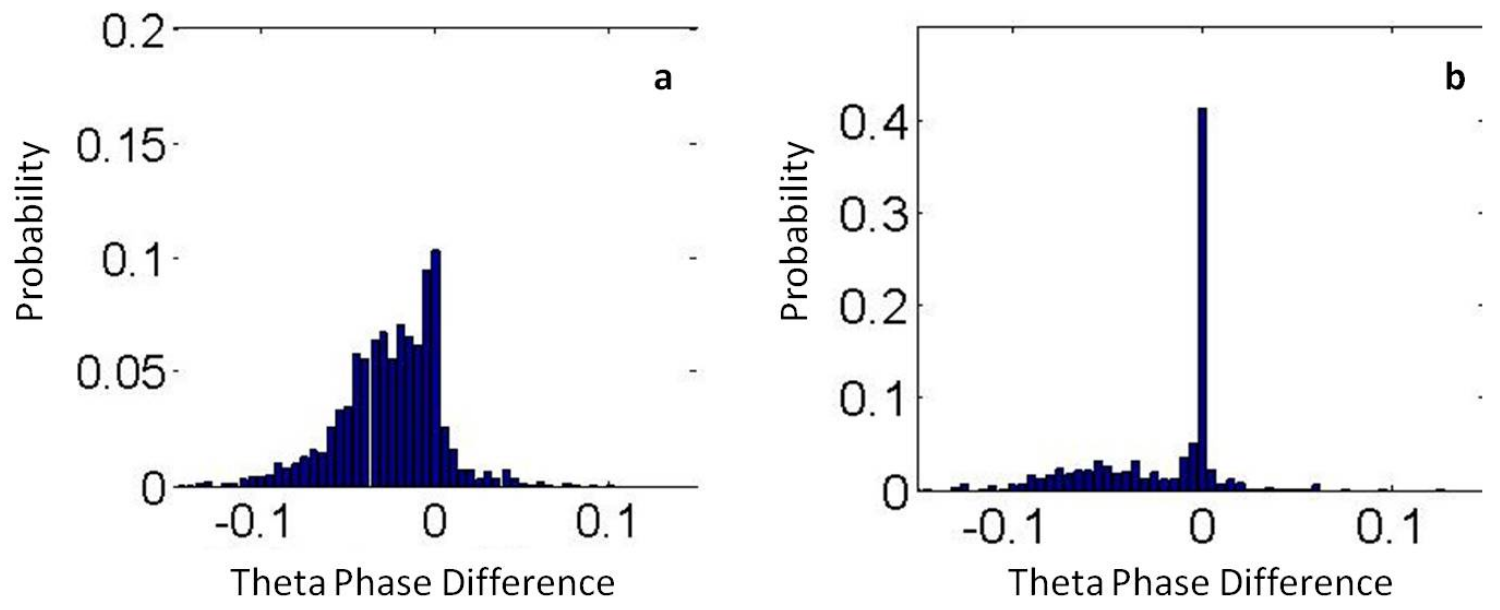

Figure 5.11: Theta phase difference distributions (a) in the early kindling stage; (b) in the fully kindled stage. Both distributions are negatively skewed, which means that the propagation pattern is sustained throughout the kindling process. 
channel 1 leads channel 2 which leads 3 which leads 4 . This pattern indicates that the seizure originates in hippocampal CA3 as a result of direct CA3 stimulation, and then propagates through the transverse extent of the monitored CA1 cell layer. The direction of propagation is from proximal to distal. This result is similar to what we have demonstrated in anesthetized rats.

The histograms of the theta phase difference distributions during both the early and final stages of kindling are shown in Figure 5.11 as comparison. Both distributions are skewed to the negative side. This implies that the propagation pattern is preserved throughout the whole kindling process.

Theta phase difference is also an indicator of how synchronized the channels are, because the distribution becomes progressively more skewed towards zero as the animal is kindled. This means that fully kindled animals are much more likely to exhibit simultaneous firing across all channels at the zero components of the theta phase distributions. Because all channels share this increased preference for, and probability of firing at, the zero component, this is evidence of increased synchronized activity.

In conclusion, theta phase analysis shows that the neuronal firings along the lamellar axis become more synchronized as kindling progresses, and that the firings propagate from the proximal to distal electrode along the lamellar axis.

\subsubsection{Oriented along the Septotemporal Axis}

Seizures $(n=145)$ were recorded within longitudinally placed microelectrode arrays in 6 rats. In Figure 5.12, the average Shannon entropy did not show a linear increase or decrease. Instead, it fluctuated up and down between 1.82 and 2.00. The Shannon entropy was $1.9 \pm 0.06$ at the early kindling stage, and $1.88 \pm 0.02$ at the fully kindled stage. There were no significant changes between the two stages ( $\mathrm{p}$ 


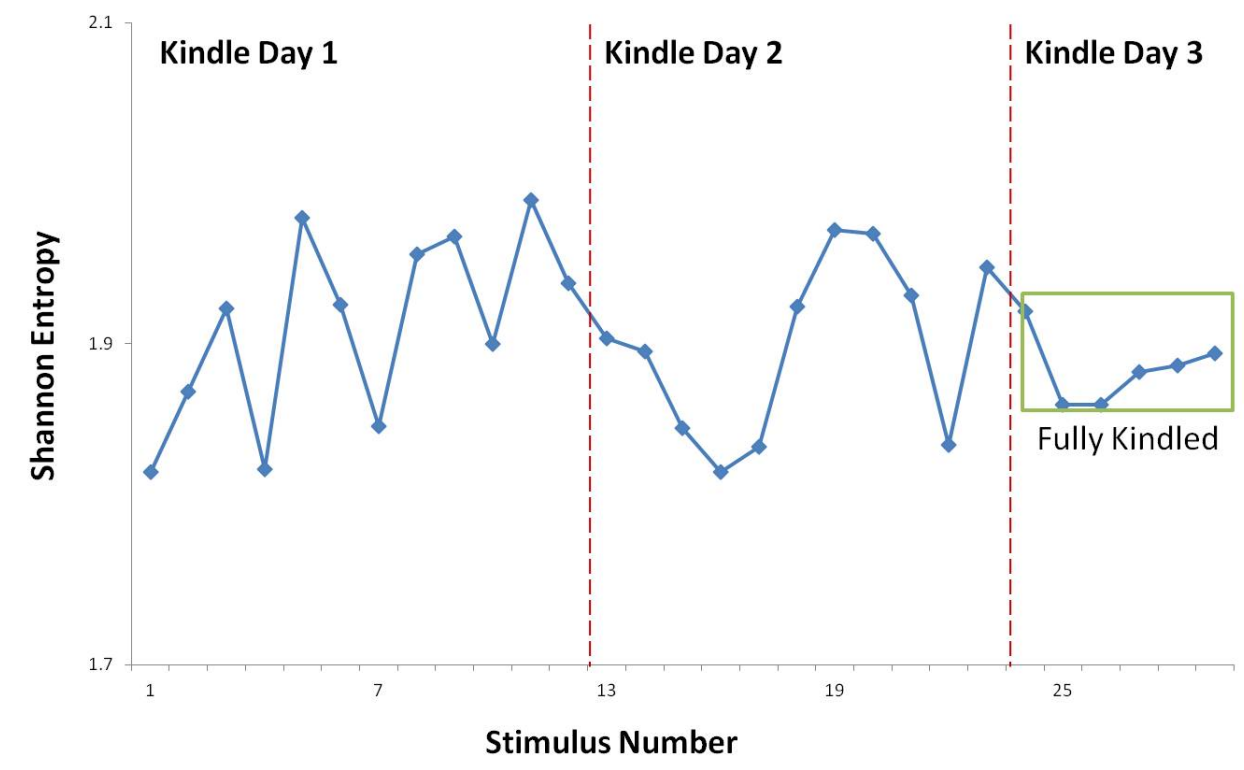

Figure 5.12: Shannon entropy fluctuated as kindling progressed along the septotemporal axis, but showed no significant changes between stages.

$>0.05$, ANOVA)(See Figure 5.13). Compared to the lamellar configuration, which was $1.95 \pm 0.03$ for stage 1 seizures and $1.51 \pm 0.05$ for stage 5 , the average Shannon entropy remained relatively high throughout kindling along the septotemporal axis. This means that the neurons did not become more synchronized as kindling progressed.

In Figure 5.13, the Shannon entropy of the four channels did not show any significant differences between each other during stage 1 or stage 5 seizures. ( $p$ $>0.05$,both ANOVA and kruskalwallis test). This indicates that the synchrony level was homogeneous in the small recording area throughout kindling. The neuron groups recorded by each electrode were not distinguishable from each other.

The theta phase distributions were spread randomly between 0 and $2 \pi$ at both the early and fully kindled stage (Figure 5.14). The neuronal firings did not interact with the underlying theta oscillations. Nor was a preferred firing phase observed in the septotemporal direction. 


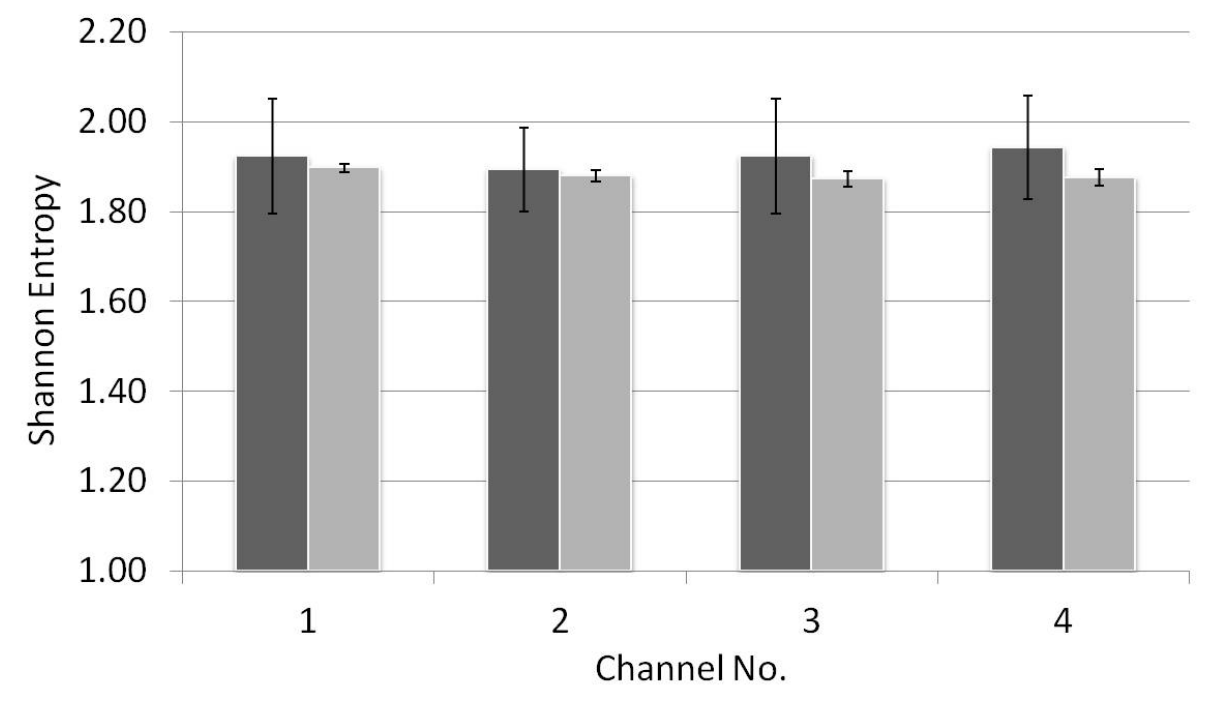

Figure 5.13: The shannon entropy of stage 1 seizures is represented by the black bar, and the stage 5 seizures are represented by the gray bar. There were no significant differences in Shannon entropy between stages or channels.

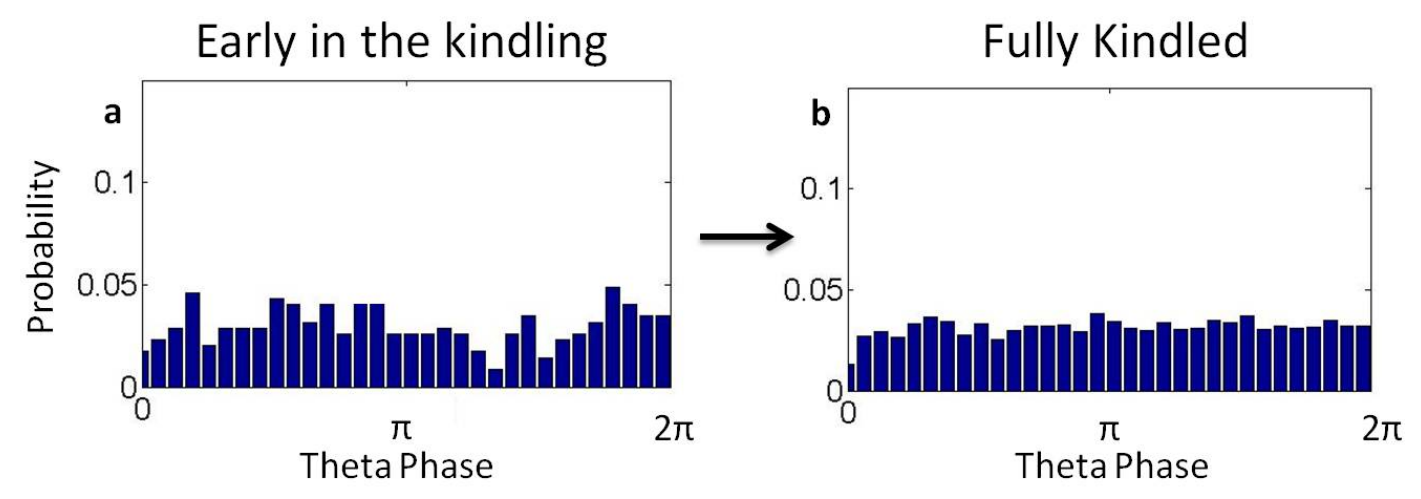

Figure 5.14: Theta phase distributions were evenly distributed between 0 and $2 \pi$, for both the early and fully kindled stages. A preferred firing phase was not seen in either stage.

As for the theta phase difference distributions, all distributions were Gaussian distribution regardless of stage (Figure 5.15). Theta phase difference distributions did not reveal any rapid propagation in the septotemporal direction.

In conclusion, Theta phase analysis shows that synchrony between the neuronal firings along the septotemporal axis did not show significant variation as kindling 


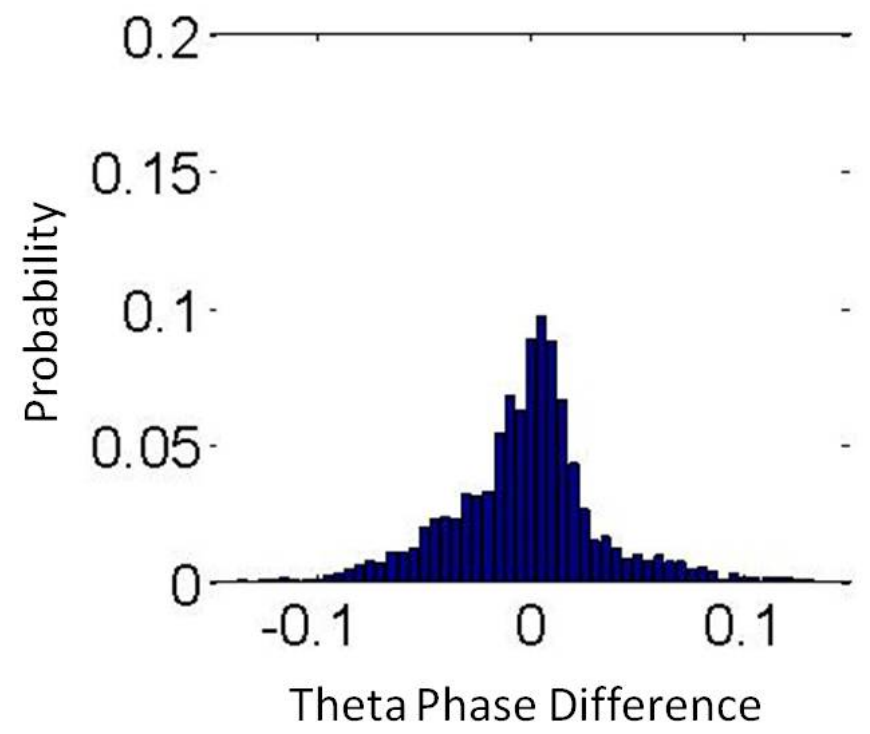

Figure 5.15: Theta phase difference distributions were Gaussian distributed around zero, which means that there was no propagation pattern in the septotemporal direction.

progressed. Also, there was no propagation pattern observed in the septotemporal direction.

\subsection{Event Synchronization}

We analyzed all of the recorded seizures, and calculated the mean and standard deviation of the event synchronization strength. It showed that neuronal firings along the lamellar axis were more synchronized (ES strength $=0.67 \pm 0.19$ ) compared to septotemporal axis (ES strength $=0.36 \pm 0.18$ ). They were significantly different from each other $(\mathrm{p}<0.001$, paired t-test $)$ as shown in Figure 5.16

Event synchronization examined the synchrony between channels of high frequency firing. In both the lamellar and septotemporal directions, channels 1 and 2, and channels 3 and 4 were more synchronized than other pairings (See Figure 5.17 and Figure 5.18). This was caused by the way the microelectrode array was 


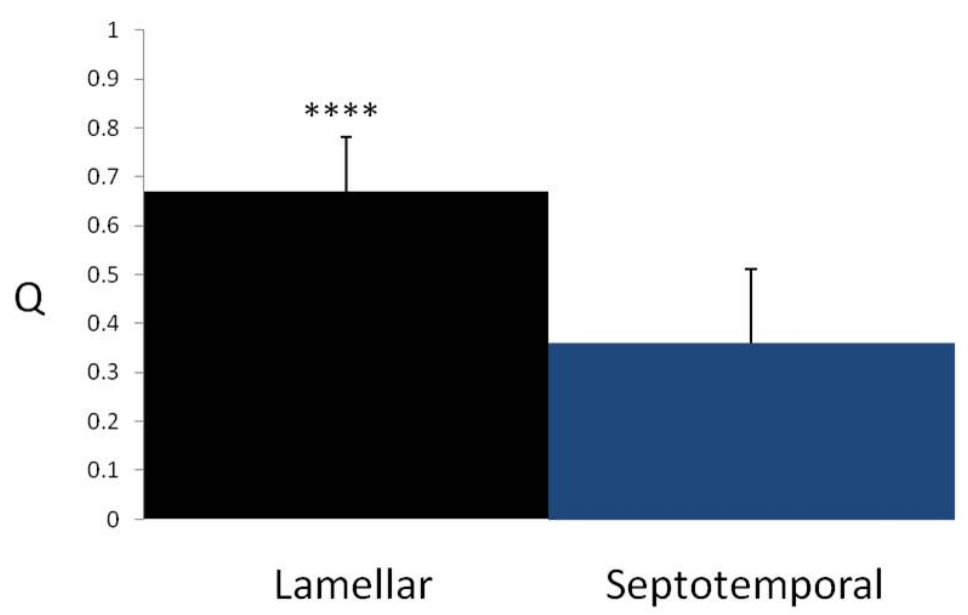

Figure 5.16: Event synchronization was significantly higher in the lamellar direction than septotemporal direction.

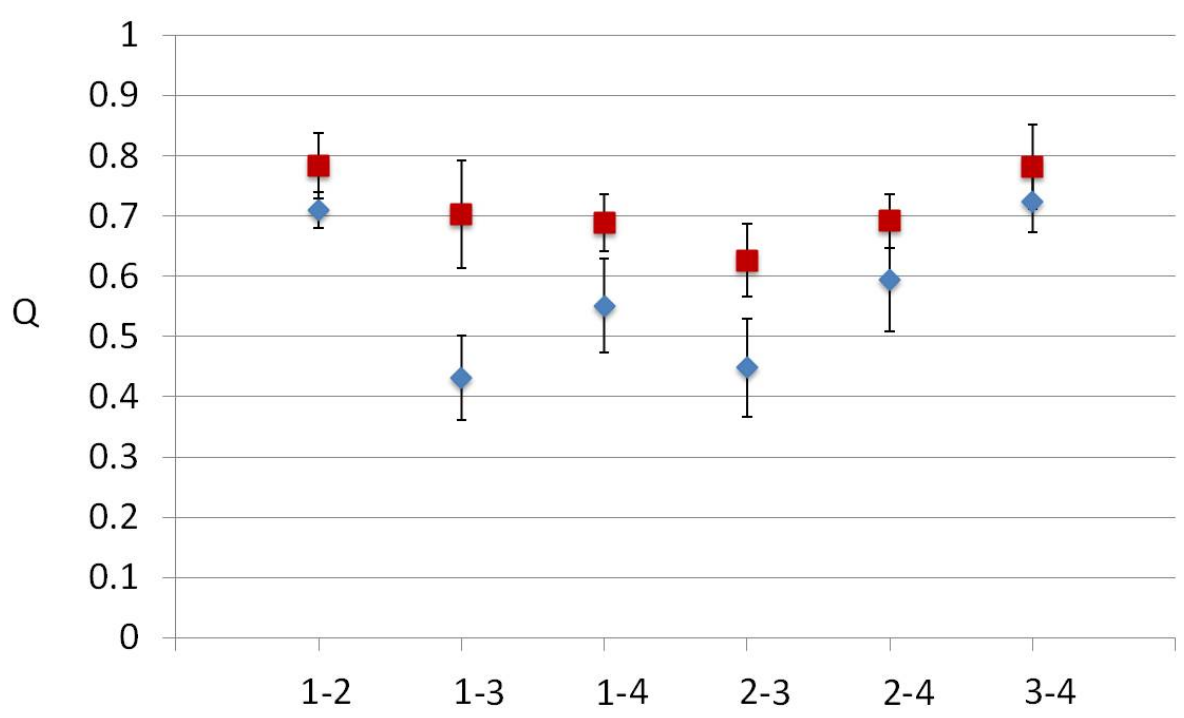

Figure 5.17: Event synchronization increased as kindling progressed along the lamellar axis. The rhombuses represent stage 1 seizures, while the squares represent stage 5 seizures.

fabricated. Channels 1 and 2, as well as 3 and 4 were closer to each other, as there was an extra layer of superglue between channels 2 and 3. Therefore the synchronization was the highest between channels 1 and channel 2, and channels 3 and channel 4 .

In the lamellar configuration (See Figure 5.17), the strength of event synchroniza- 


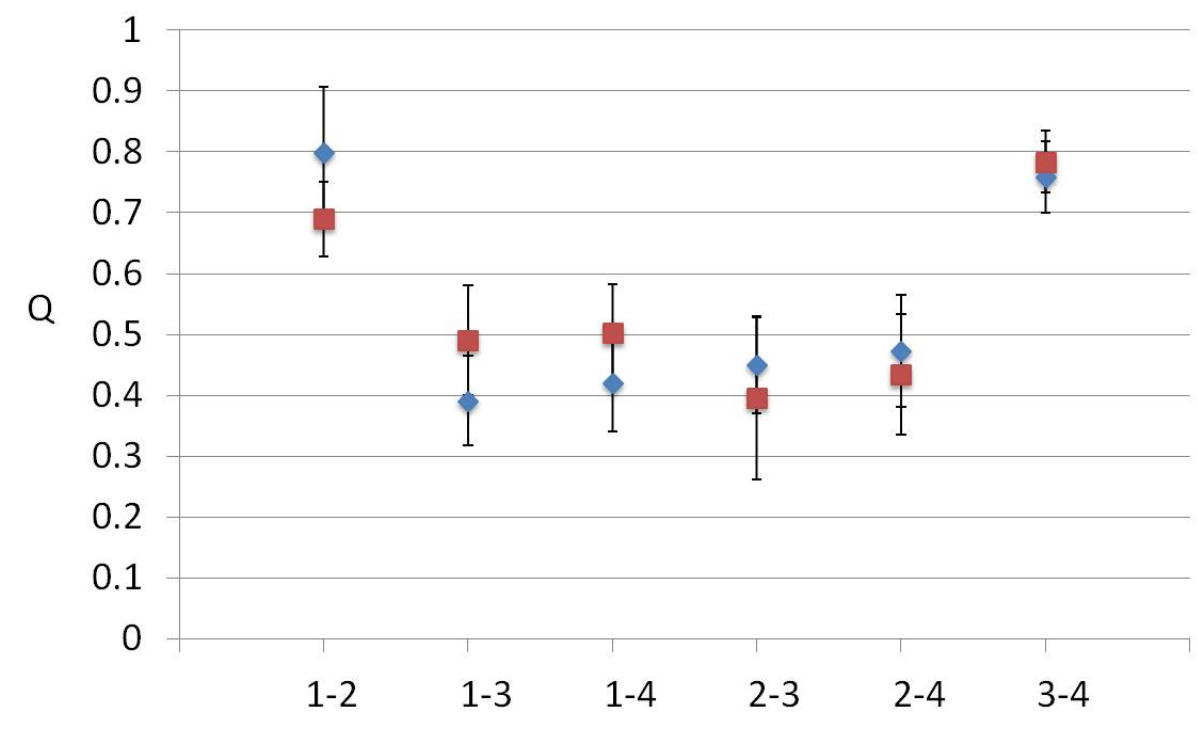

Figure 5.18: Event synchronization showed no significant difference as kindling progressed along the septotemporal axis. The rhombuses represent stage 1 seizures, while the squares represent stage 5 seizures.

tion in pairs $1-3,1-4$, and 2-3 during the early stages of kindling were significantly different from that of the fully kindled stage ( $p<0.05$, ANOVA). In the septotemporal direction, no significant differences in synchrony strength were seen between the different stages of kindling (Figure 5.18).

\subsection{Conclusion}

In this chapter, we investigated the dynamic neuronal synchrony in a two dimensional surface in hippocampal CA1 cell layer during electrical kindling in awake rats. The rapid kindling model was deliberately picked for this study as it enabled us to study the development of synchronization processed as synchronization across successive seizure stages.

First, two commonly used seizure severity measurements were utilized, BSS and ADD. The former, the BSS, divides the seizures into five stages based on 
animal behavior during evoked seizures. We were able to show that the rapid kindling protocol successfully kindled the rats, resulting in advancing behavior scores. While the latter, the ADD, shows a rapid increase during the first day of kindling. It increases at a slower rate for the rest of the kindling.

Three synchronization measurements were applied to the high frequency firing data: cross correlation, theta phase synchronization, and event synchronization. Cross correlation shows that the high frequency firings were highly correlated along the lamellar axis, but not the septotemporal. Both theta phase synchronization and event synchronization quantitatively demonstrate that neuronal synchrony along lamellar axis increased in the small monitored area as kindling progressed; whereas no significant variations in synchrony were observed along the septotemporal axis. Event synchronization demonstrated that firings along the lamellar axis were more synchronized compared to those of the septotemporal axis.

Theta phase synchronization also revealed a distinct propagation pattern in the hippocampal CA1. High frequency firing propagated from proximal to distal along the lamellar axis; while no propagation pattern was observed along the septotemporal axis. 


\section{Chapter 6}

\section{Synchronization during}

\section{Anti-epileptic Drug Treatment}

The purpose of this study was to test the effects of two anti-seizure drugs on synchrony during electrically evoked seizures in freely moving rats. EEG recording was accomplished via a microelectrode array implanted along the lamellar axis in the hippocampal CA1 cell layer. This was done in a total of 8 animals across two treatment groups (Phenytoin, $n=4$; Levetiracetam, $n=4$ ). To ensure that the animals were fully kindled before drug treatment testing, the animals were exposed to the stimulus until a minimum of three stage 5 behavioral seizures had been recorded. After the injection, the same stimulus was delivered 8 more times, once every 30 minutes for 4 hours (when the recording ended) to the CA3 region of the hippocampus. Synchrony was measured in the same manner as before. 


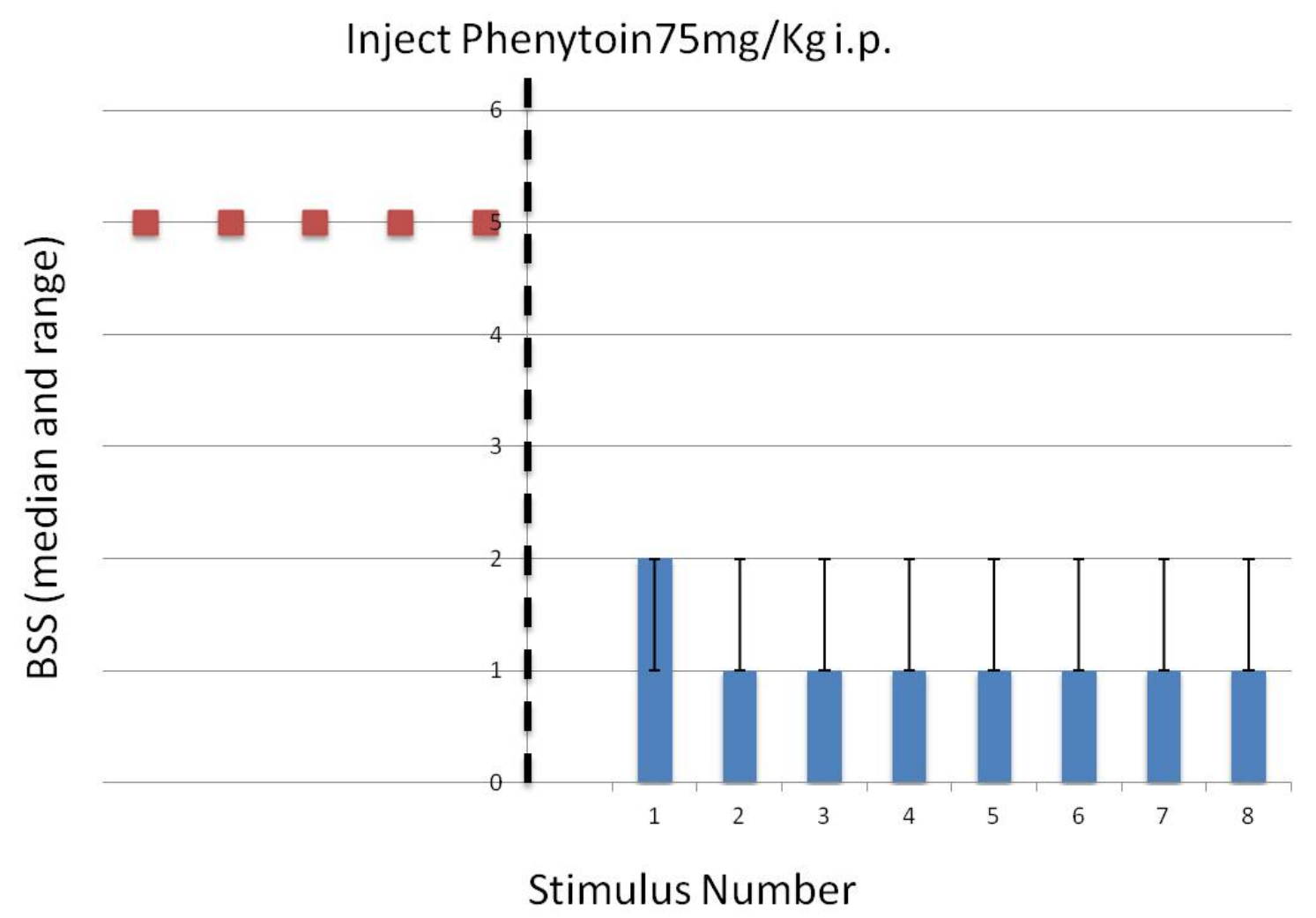

Figure 6.1: After the phenytoin treatment, BSS dropped from stage 5 to stage 1 or 2 .

\subsection{Phenytoin Treatment}

\subsubsection{Behavioral Seizure Scores and After-discharge Duration}

In order to quantify the severity of evoked seizures, BSS and ADD were recorded.

Thirty minutes after the phenytoin injection, behavioral seizure stage dropped from stage 5 to stage 1 or 2. As shown in Figure 6.1, BSS stayed at this reduced level for 4 hours (until the end of the recording).

The after-discharge duration also showed a similar decrease (Figure 6.2). The mean ADD was $39.6 \pm 9.1$ seconds for fully kindled seizures. Thirty minutes after the phenytoin treatment, the ADD declined significantly to $7.8 \pm 3.2$ seconds. This level of seizure duration was maintained over the remaining 4 hours of the 


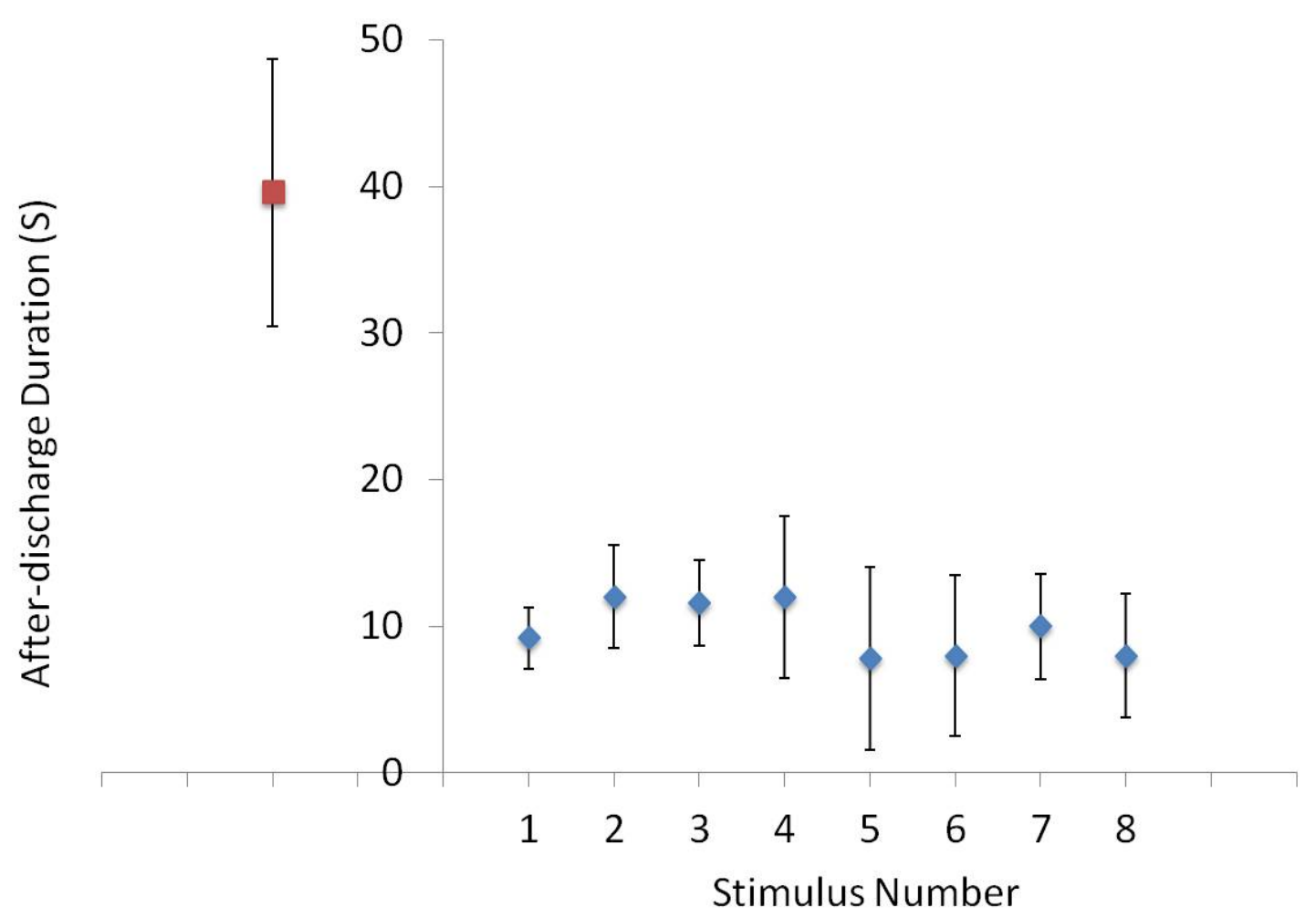

Figure 6.2: After the phenytoin treatment, ADD was reduced to a lower value.

recording. It did not increase with successive applications of the stimulus.

Both BSS and ADD demonstrate that the severity of seizures reduces after the phenytoin treatment, and does not recover within 4 hours.

\subsubsection{Spike Rate}

The first thing we looked at with the high frequency firing data was the firing frequency. The spike rate of the seizures before treatment was significantly different from that of the seizures afterwards $(\mathrm{p}<0.05$, Kolmogorov-Smirnov test) (See Figure 6.3). The low frequency component (under $10 \mathrm{~Hz}$ ) was significantly reduced as a percentage of the seizure's total frequency construction after the phenytoin treatment. Correspondingly, the high frequency component (above $300 \mathrm{~Hz}$ ) gained 

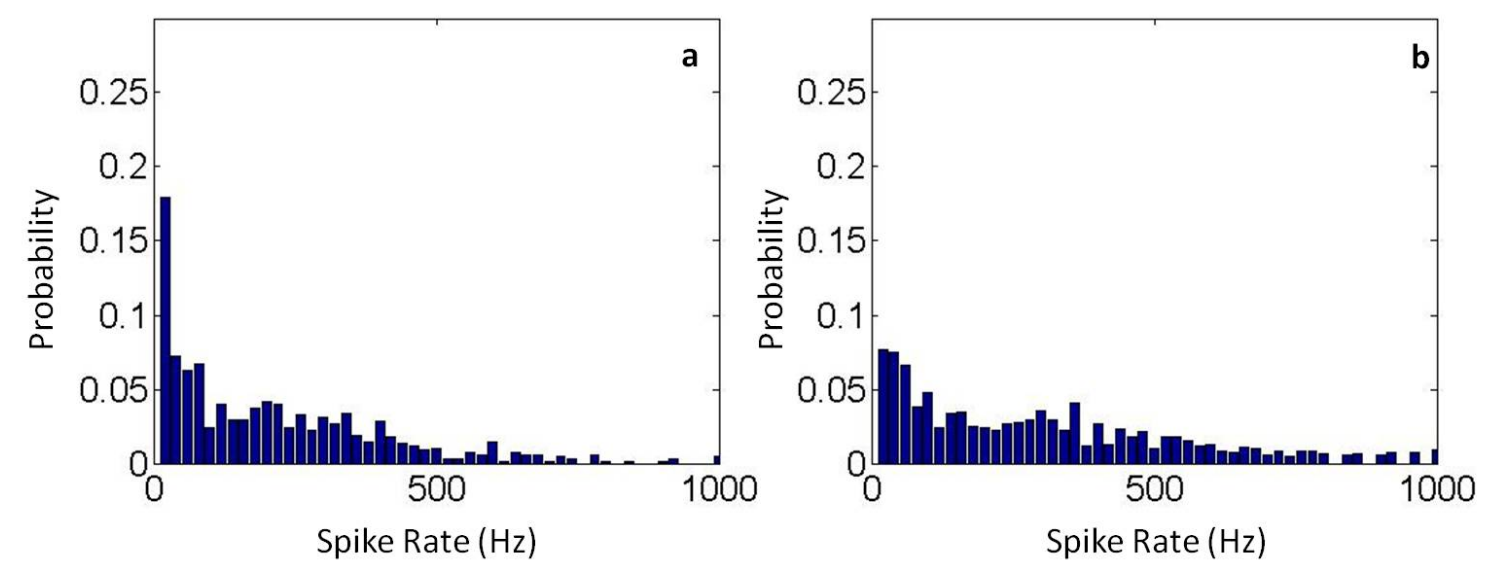

Figure 6.3: Spike rate before(a) and after(b) the phenytoin treatment.

percentage share. These low frequencies typically occurred in the time periods between bursts, while the high frequencies tended to occur within. Therefore these frequency shifts demonstrate an increased preference for bursting activities during seizures in the phenytoin treated rats.

\subsubsection{Theta Phase Synchronization}

Theta phase analysis robustly demonstrates that neuronal synchrony decreases after phenytoin treatment. Order, and therefore synchrony, was quantified by calculating the Shannon entropy of the theta phase distributions. The Shannon entropy of the evoked seizures was significantly different from that of the stage 5 seizures ( $\mathrm{p}<0.05$,ANOVA), for the first 2 hours after phenytoin treatment (See Figure 6.4). The mean Shannon entropy increased for the first one and a half hours, which indicates that after the treatment, the evoked seizures became significantly less synchronized than the stage 5 seizures. After 2 hours, the Shannon entropy recovered to the pre-injection level.

We compared the synchrony change with BSS and ADD changes. The theta phase synchronization returned to pre-treatment level before BSS and ADD. This 


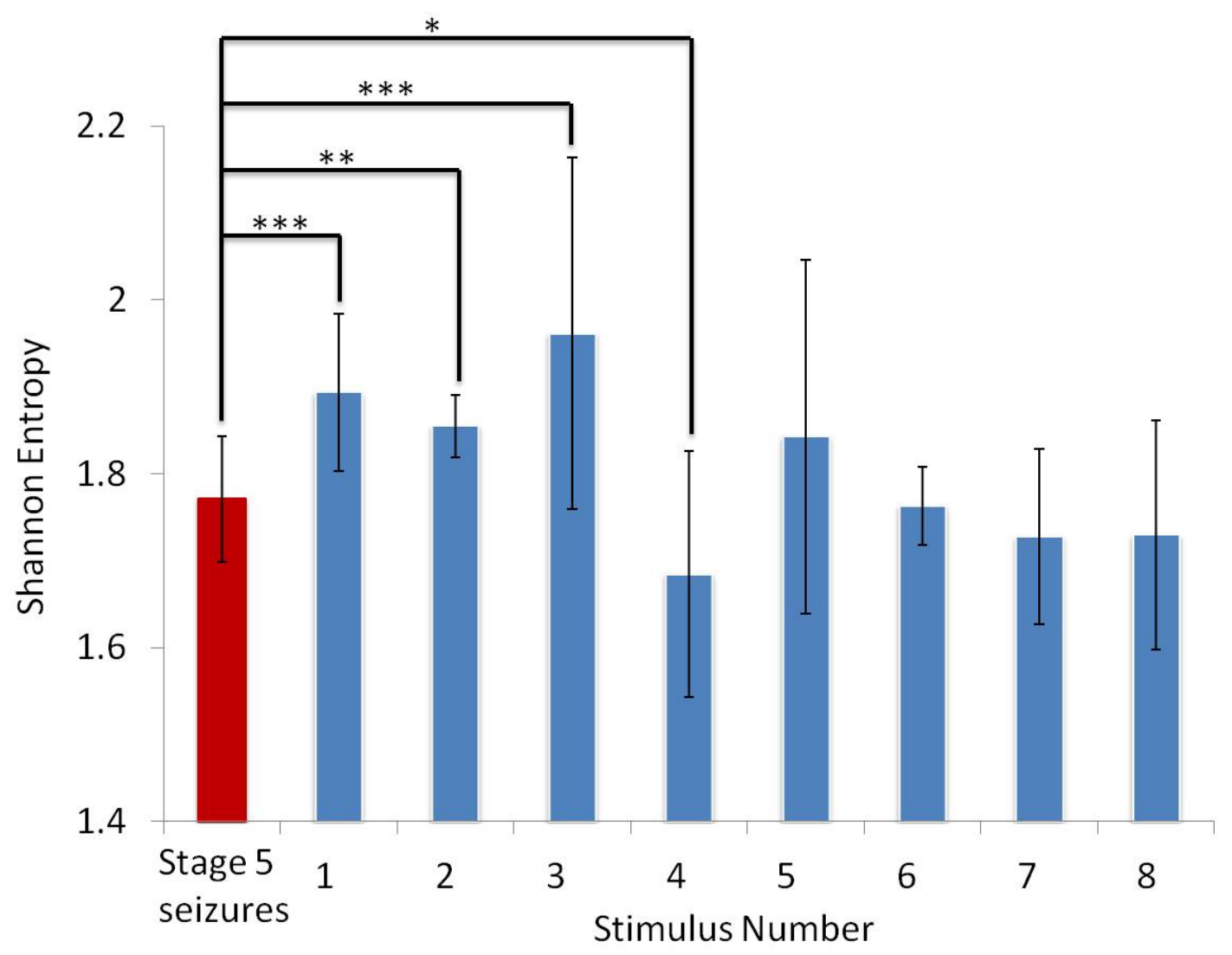

Figure 6.4: Shannon entropy increased after the treatment for the first one hour and half and returned to the pre-injection level after 2 hours. The red bar represents the Shannon entropy of the stage 5 seizures, while the blue bars represent the entropy of the evoked seizures after the phenytoin treatment.

indicates that the seizure behavior and duration are suppressed by phenytoin. Even when the behavioral score and after-discharge duration remain at a low value, the synchronization between the firings starts to come back.

The preferred firing phase value, which occurred around the trough of theta oscillation, was lost after the phenytoin treatment (See Figure 6.5), and the theta phase distribution became evenly spread between 0 and $2 \pi$ as well. This indicates that synchrony was destroyed by the phenytoin treatment. However, the preferred firing phase returned 2 hours later as synchrony returned to the pre-injection levels. 

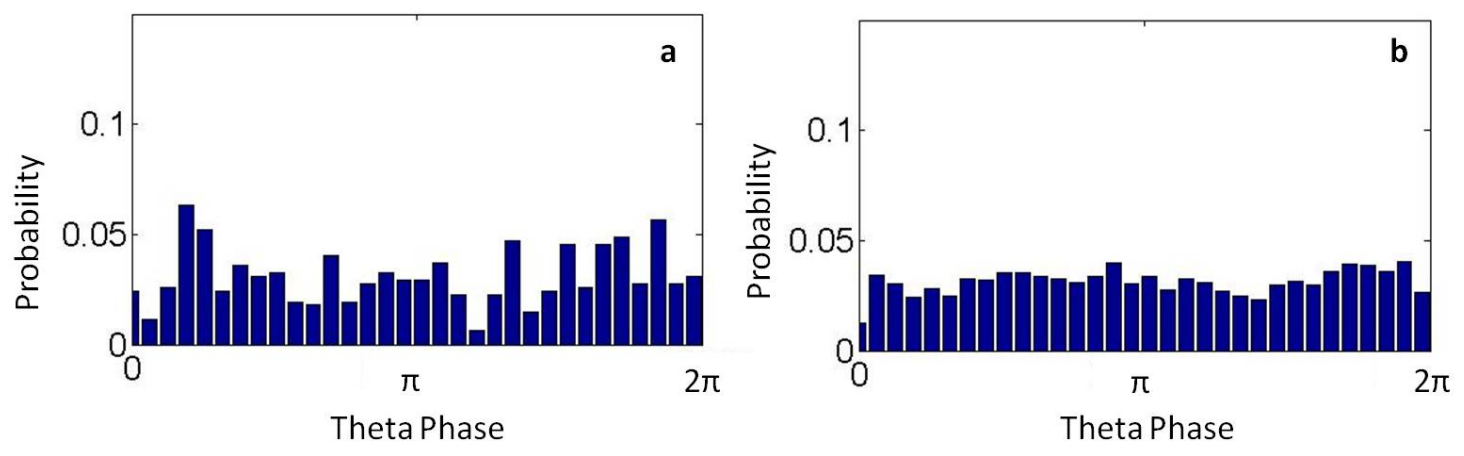

Figure 6.5: Theta phase distribution (a) before and (b) after phenytoin injection. The preferred firing phase was lost after the phenytoin treatment.

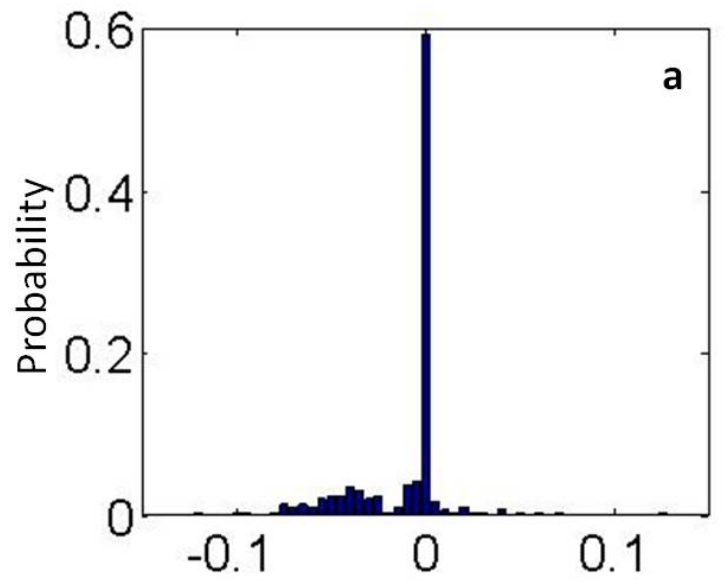

Theta Phase Difference

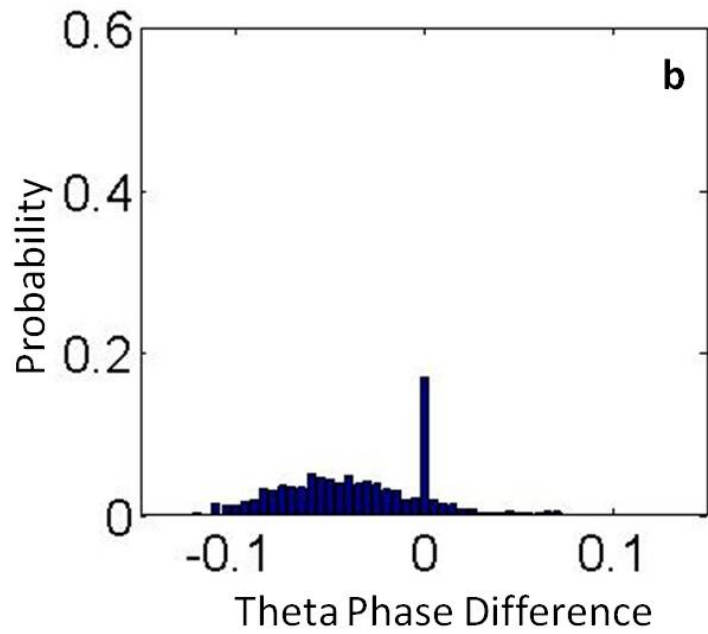

Figure 6.6: Theta phase difference distribution before(a) and after(b) phenytoin treatment. Both distributions were negatively skewed. Also, the population around 0 was reduced in the distribution after the phenytoin injection.

Theta phase differences were calculated between all possible channel pairings (a total of 6). Firstly, all of these distributions were negatively skewed before and after the phenytoin treatment (see Figure 6.6). The median and mean were negative for all distributions, which indicate that the firing recorded by one channel leads the other channel. The firings propagated through the transverse extent of the monitored CA1 cell layer, from proximal to distal, which suggests that phenytoin does not affect this propagation pattern. Secondly, theta phase distribution showed 


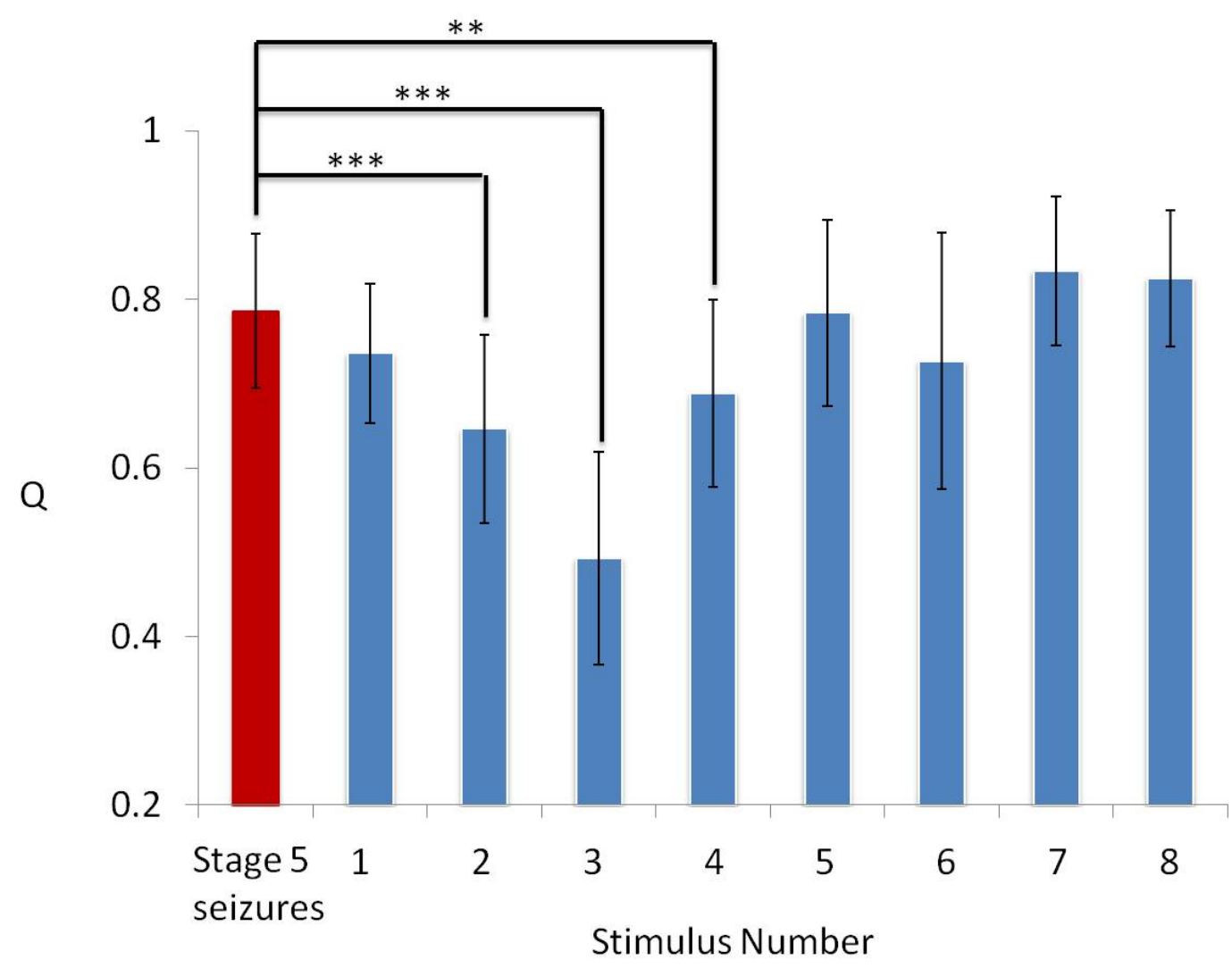

Figure 6.7: Event synchronization before(a) and after(b) phenytoin injection. The synchrony decreased after treatment for the first two hours, then returned to the pre-injection level. The red bar represents the event synchronization strength of the stage 5 seizures; while the blue bars represent synchronization of levels of successive, post phenytoin treatment evoked seizures.

a reduced population around 0 after the phenytoin injection, likely because there was a longer time delay between recording sites due to the decrease in neuronal synchronization.

\subsubsection{Event Synchronization}

In Figure 6.7, thirty minutes after phenytoin treatment, the event synchronization strength was not significantly different from that of the stage 5 seizures $(\mathrm{p}=0.097$, ANOVA). However, by 1-2 hours post injection, the change had became 
significant ( $\mathrm{p}<0.01$, ANOVA). The mean synchrony attenuated and reached its lowest value at about 1.5 hours post injection. Starting around 2 hours post injection, synchrony values slowly began to return to pre-injection levels. This return trend was also seen in the theta phase analysis.

Both theta phase synchronization and event synchronization showed that synchrony decreased within the first 2 hours of phenytoin injection and returned back to pre-injection levels within 4 hours of treatment. Conversely, BSS and ADD remained at significantly reduced levels for the duration of the recording.

\subsection{Levetiracetam Treatment}

\subsubsection{Behavioral Seizure Scores and After-discharge Duration}

As with the phenytoin treated animals, the animals that received an injection of levetiracetam $(n=4)$ displayed a marked and rapid decrease in behavioral seizure stage, falling from stage 5 at the time of injection, to stage 2 at the next application of the stimulus (30 minutes later) (See Figure 6.8). But while phenytoin treated animals maintained these low behavioral seizure stages for the remainder of the recording, the BSS of levetiracetam-treated animals began to increase approximately one hour after injection. By the end of the recording, 3 out of 4 rats had recovered to stage 5 behavioral seizures.

The ADD also decreased significantly after the LEV treatment (Figure 6.9). The mean ADD was $91.6 \pm 20.3$ seconds for stage 5 seizures. Thirty minutes after LEV injection, the mean ADD reduced to $50.1 \pm 13.7$ seconds. However, unlike BSS, ADD remained at reduced levels, averaging $45.3 \pm 17.2$ seconds at the end of the recording, 4 hours later. 
CHAPTER 6. SYNCHRONIZATION DURING ANTI-EPILEPTIC DRUG

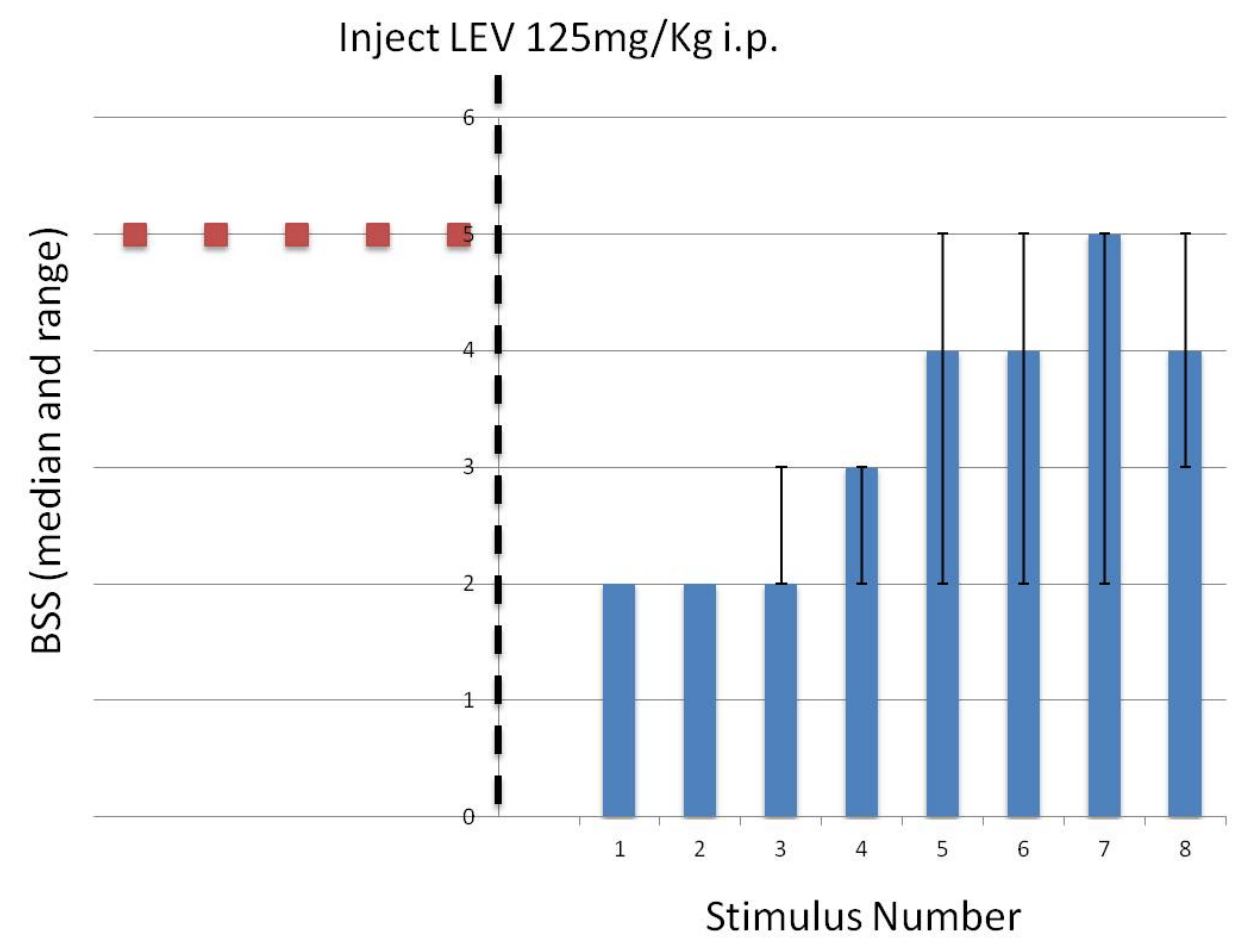

Figure 6.8: After the LEV treatment, BSS dropped from stage 5 to stage 2 before recovering back to stages 4 and 5 .

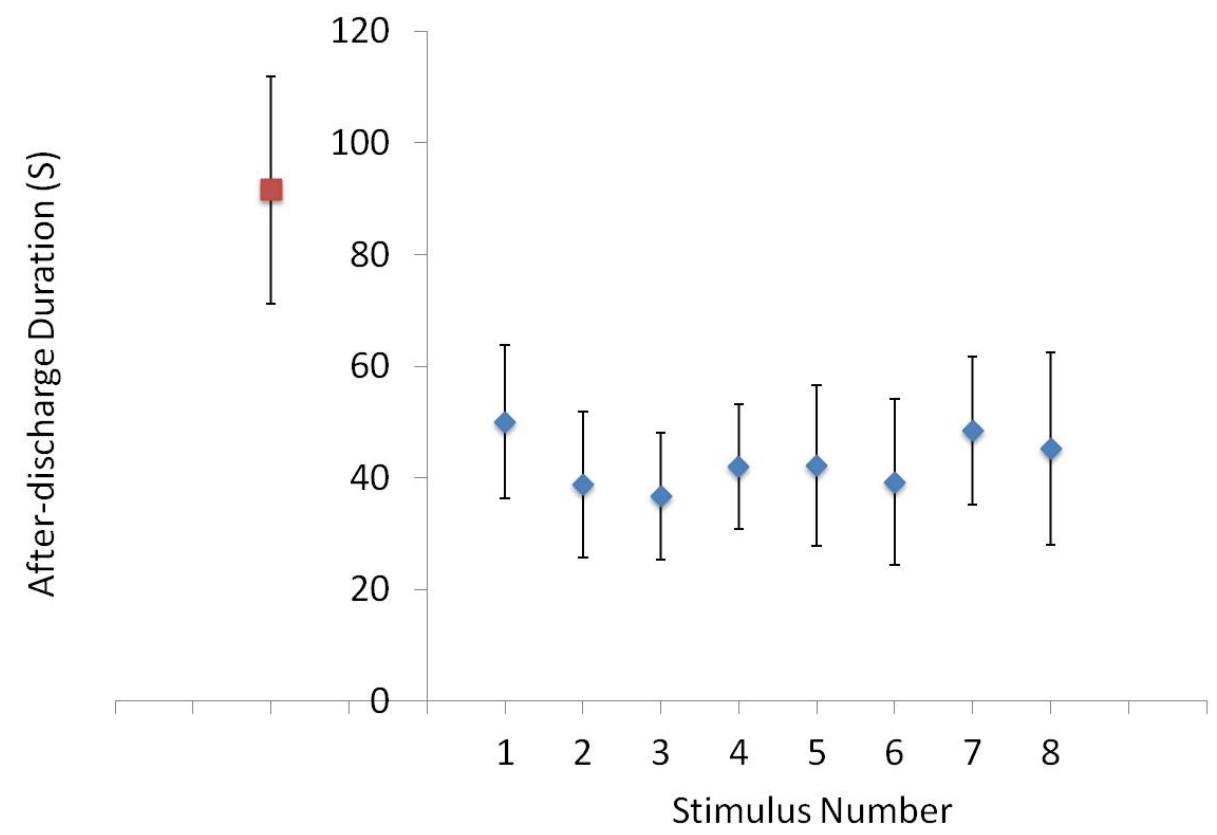

Figure 6.9: After the LEV treatment, ADD fell to lower values. 

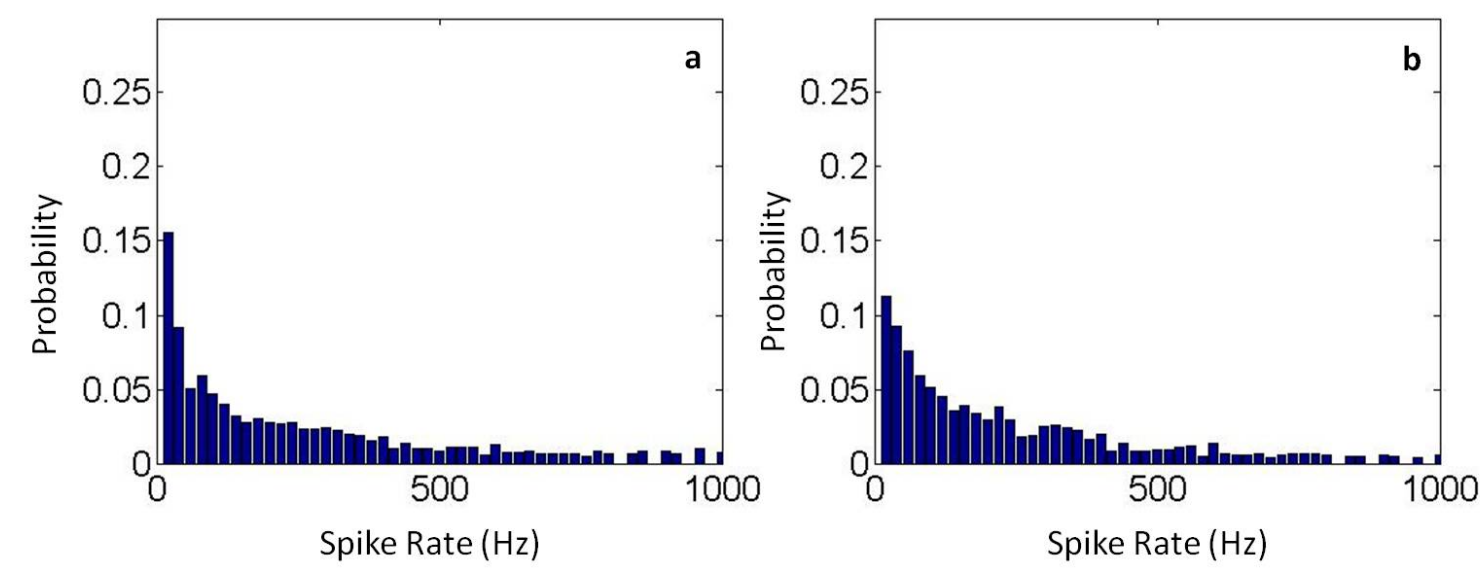

Figure 6.10: Spike rate before(a) and after (b) the LEV treatment.

\subsubsection{Spike Rate}

The firing frequency analysis yielded similar results to that of the phenytoin experiments. That is, treatment with LEV significantly altered the spike frequency composition of evoked seizures ( $\mathrm{p}<0.05$, Kolmogorov-Smirnov test) (Figure 6.10). The low frequency component (under $10 \mathrm{~Hz}$ ) was reduced by the LEV treatment, while the high frequency component (above $300 \mathrm{~Hz}$ ) experienced an increase. This is the same frequency shift observed in the phenytoin treated rats.

\subsubsection{Theta Phase Synchronization}

Theta phase analysis robustly demonstrates that neuronal synchrony decreases after LEV treatment. Shannon entropy was calculated to quantify the order in theta phase distribution. The Shannon entropy of the evoked seizures was significantly different from that of the stage 5 seizures ( $p<0.001$, ANOVA), for the first 2 hours after LEV, as shown in Figure 6.11. The mean Shannon entropy increased for the first one and a half hours after the treatment, which indicates that the evoked seizures become less synchronized than the stage 5 seizures. However, this was 


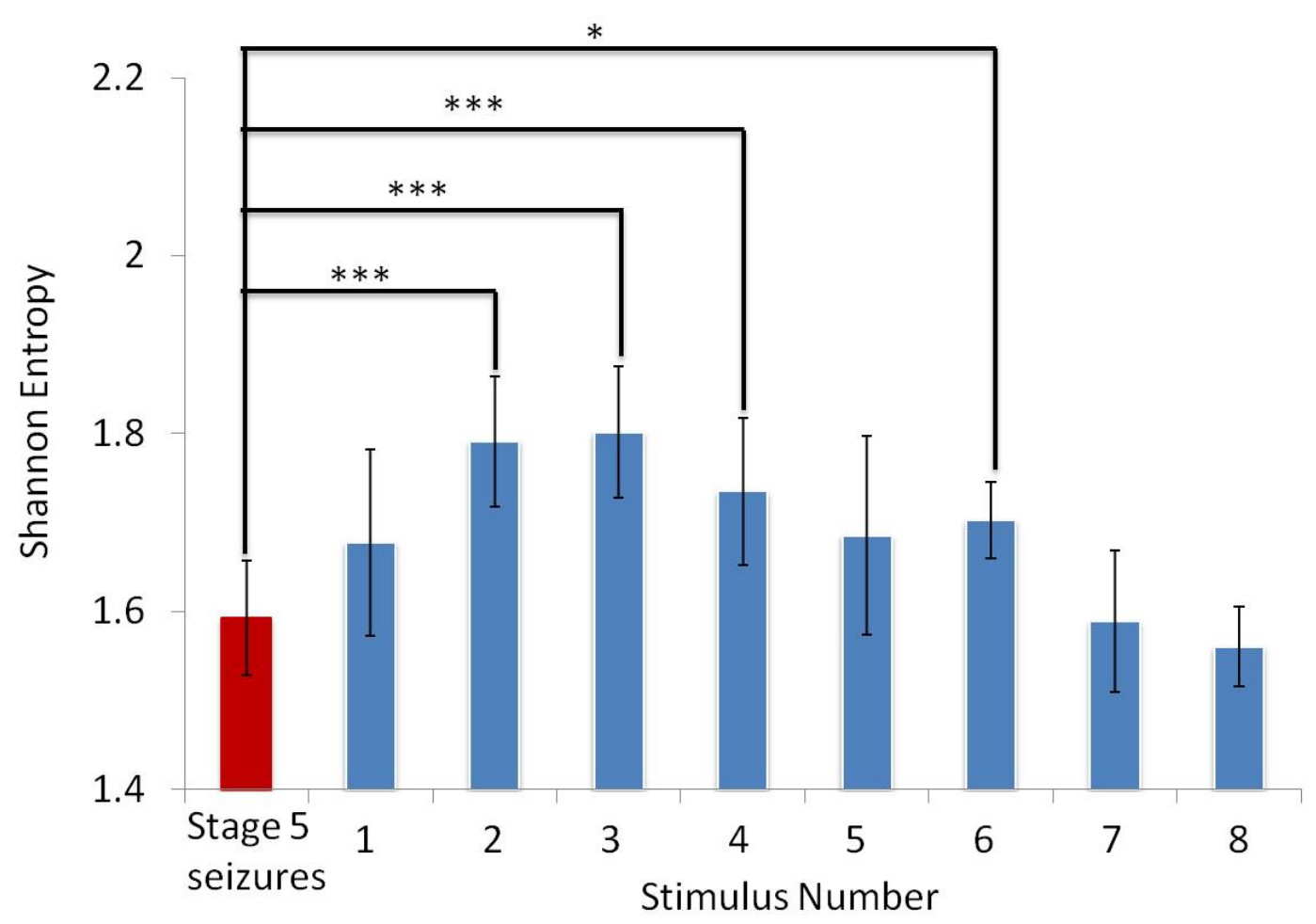

Figure 6.11: Shannon entropy increased after the treatment for the first one and a half hours before returning to the pre-injection levels. The red bar represents the Shannon entropy of the stage 5 seizures, while the blue bars represent the entropy of the evoked seizures after the LEV treatment.

temporary, as the mean Shannon entropy started to decrease and returned to the pre-injection level after 3 hours.

We compared the synchrony change with BSS and ADD change. The theta phase synchronization and BSS returned to pre-treatment level around the same time, while ADD remained suppressed by LEV.

The preferred firing phase value, which occurs around the trough of the theta oscillations, was lost after LEV treatment (Figure 6.12). The theta phase distribution became evenly spread between 0 and $2 \pi$ after the injection. This indicates that synchrony was destroyed by the LEV treatment. The preferred firing phase returned 3 hours after injection as synchrony recovered to the pre-injection levels. 


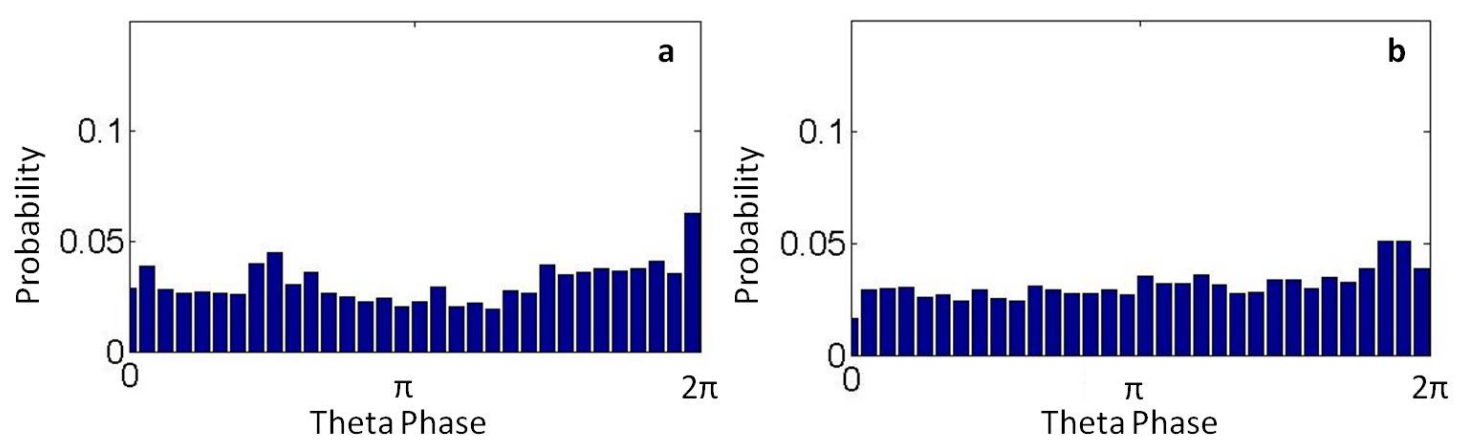

Figure 6.12: Theta phase distribution before(a) and after(b) after LEV injection. The preferred firing phase was initially lost aft the LEV treatment, although it returned 3 hours after injection.
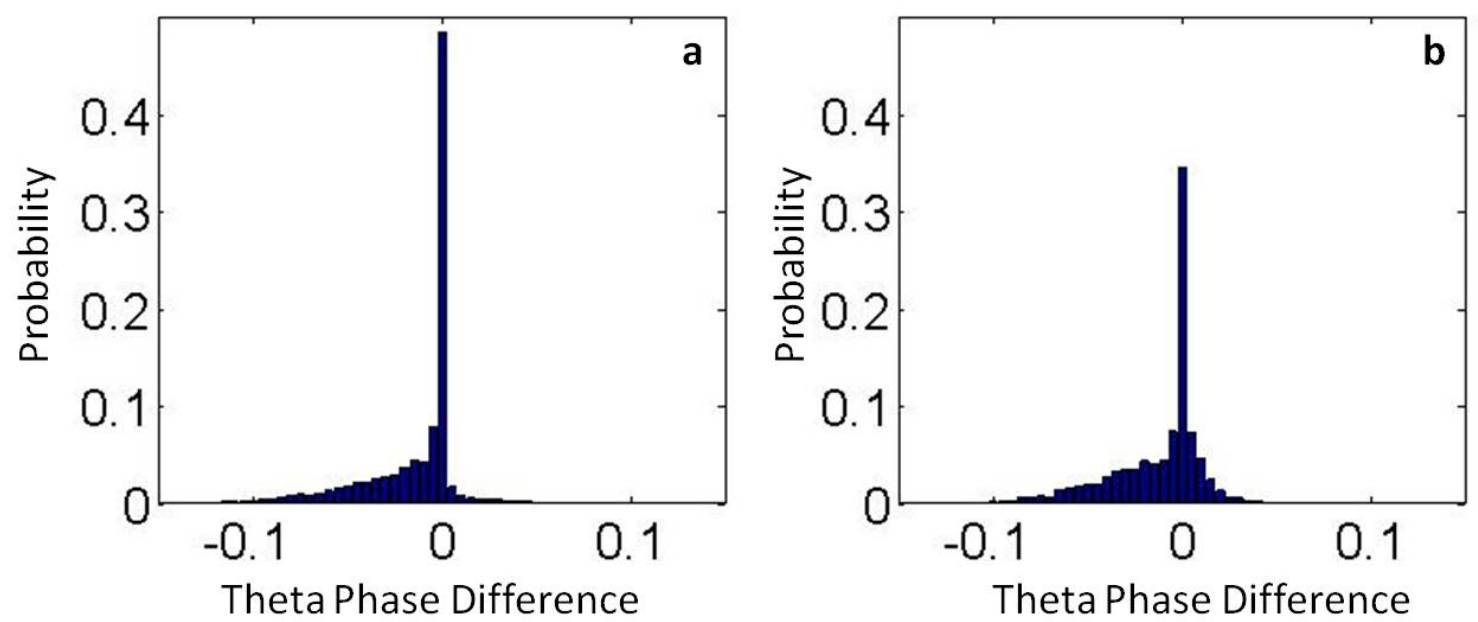

Figure 6.13: Theta phase difference distribution before(a) and after(b) the LEV treatment.

Theta phase differences were calculated between all possible channel pairings. All six distributions were negatively skewed before and after LEV injection. It indicated that the propagation pattern was not affected by the administration of LEV, and that high frequency firing propagated from proximal to distal along the lamellar axis. The theta phase difference distribution did not show a significant difference before and after the LEV treatment (Figure 6.13). Nor was there a significant difference between the mean, median or skewness of the distribution ( $\mathrm{p}$ $<0.05$, ANOVA). This stands in stark contrast to the phase difference distributions 


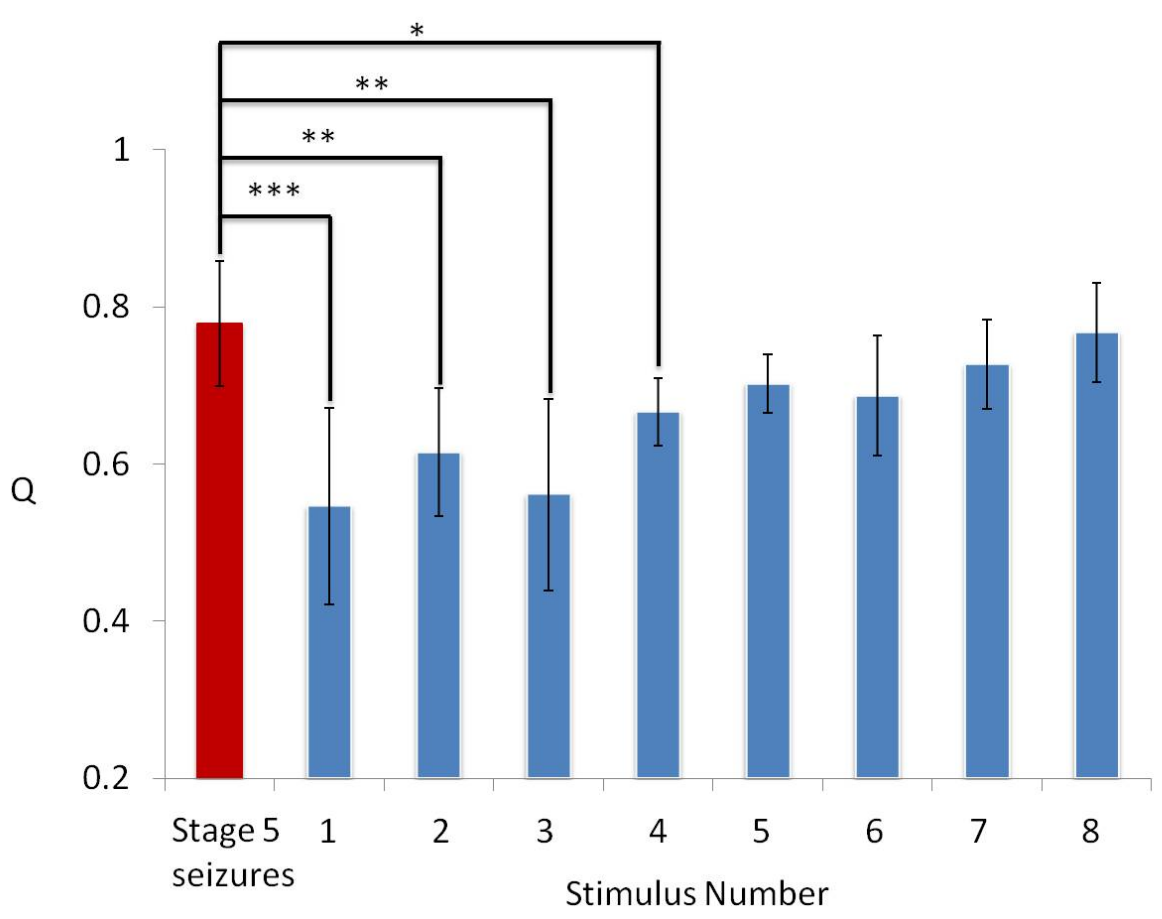

Figure 6.14: Event synchronization before(a) and after(b) the after LEV treatment. The synchrony decreased after the treatment for the first two hours and then returned to pre-injection levels. The red bar represents the event synchronization strength of the stage 5 seizures; while the blue bars represent after LEV injection.

of the phenytoin-treated animals, where there were significant differences between the before and after of treatment of the phase difference distributions.

\subsubsection{Event Synchronization}

In Figure 6.14, event synchronization fell to a lower value over the course of the 2 hours immediately following the LEV treatment, before slowly returning to its pre-injection value.

The initial decrease in the strength of event synchronization $(Q)$ was significant $(p$ $<0.05$, ANOVA) when compared to the behavioral stage 5 seizures, and it remained so for the duration of the first two hours post treatment. After which Q returned to 
its pre-injection levels. This return to baseline is also apparent in the theta phase analysis.

\subsection{Conclusion}

In this chapter, we present our findings from investigating dynamic neuronal synchrony before and after two commonly used anti-epileptic drugs, phenytoin and levetiracetam. Theta phase synchronization and event synchronization were used to quantify the synchrony of neuronal firing along the lamellar axis before and after drug treatment. Both synchronization measurements demonstrated that synchrony decreased within the first 2 (approximately) hours of the phenytoin/levetiracetam treatment before returning to pre-injection levels. The disruption of synchrony leads to the disruption of the preferred firing phase for both treatments. The preferred firing phase comes back as synchrony returns to its pre-injection levels. Neither antiseizure drug affected the propagation pattern along the lamellar axis, from proximal to distal. 


\section{Chapter 7}

\section{Discussion}

The goal of this study was to characterize the dynamic properties of neuronal firings during epileptogenesis and seizures, and to quantify the synchrony of high frequency firing by a linear measurement of synchrony, cross correlation, and two non-linear measurements, theta phase synchronization and event synchronization. We described and characterized the complex spatial-temporal synchrony structure of evoked seizures in both anesthetized and awake rats. We also tested the effects of two antiseizure drugs, phenytoin and levetiracetam, on seizure severity and neuronal synchrony.

Neuronal activity during a seizure is complex, high-dimensional, nonlinear, nonstationary, and noisy [111]. It has been hypothesized that seizures are the result of hypersynchronous firing of neurons [56]. However, recent studies have suggested that seizures may involve both synchronized and desynchronized neuronal dynamics $[2,58,60-64]$. It is still unclear whether a seizure can be described as a completely synchronized state, or if the mechanisms underlying generation, maintenance and propagation are more complex. This study yielded some interesting results. We found that in the lamellar direction, neurons are maximally correlated 
during the middle phase of a seizure, while both the beginning and the end of a seizure are less well-correlated. This indicates that neurons are not synchronized at the same level throughout evoked seizures. Also, we found that neurons were more correlated along the lamellar axis than those along the septotemporal axis. This suggests that the firings of neurons were not homogeneously synchronized across locations. These results imply that in contrast to the commonly held view, seizures are not hypersynchronized activities, not in time and space, and lamellar configuration might still hold as a useful concept in synchronization analysis.

The lamellar hypothesis argues that hippocampal cells are activated in a stripelike fashion, and that the principal excitatory pathways of the hippocampus are organized in a lamellar fashion [50]. Recently, with the development of intact in vitro hippocampal preparation, the intra-hippocampal epileptiform activity was shown to propagate in both the lamellar and septotemporal directions $[1,5,52]$. However, the lamellar hypothesis had not previously been tested in whole animals. To further study seizure propagation in the hippocampal CA1 cell layer, we investigated neuronal synchrony in both lamellar and septotemporal directions in awake rats. The complex structure underlying seizure propagation was explored by theta phase synchronization. We discovered that neuronal firings in the hippocampal CA1 propagate along the lamellar axis, which is from proximal to distal in the hippocampus CA1 region. Proximal CA1 is the part of CA1 that is close to CA3. This pattern indicates that seizures originate in the hippocampal CA3 as a result of direct CA3 stimulation, and then propagate through the transverse extent of the monitored CA1 cell layer. However, no propagation pattern was observed along the septotemporal axis. These findings suggest that neurons primarily propagate along the lamellar axis during evoked seizures. Even more interestingly, we revealed that the propagation pattern is preserved in the lamellar direction throughout 
the kindling process, while no rapid propagation pattern is observed, or emerges, in the septotemporal direction. Therefore, for the first time, a two dimensional propagation structure of neuronal firing was discovered during kindling. We conclude that the lamellar organization remains a useful concept for understanding the propagation of hippocampal neuronal firings during kindling.

Kindling is known to increase the afterdischarge duration and contribute to epileptogenesis [112]. Even though kindling does not cause a large loss of neurons in the hippocampus [47], it induces neuronal circuit reorganization (mossy fibers sprouting from pre-existing axons within the hilus of the dentate gyrus and along the dorsal-ventral hippocampal axis) and a variety of cellular alterations [45]. However, there has not been any study showing whether or how the structure of synchrony changes during kindling in awake animals. We studied the spatial-temporal structure of synchrony during the epileptogenesis process in both the lamellar and septotemporal directions. Our study revealed that the synchrony level along the lamellar axis increases as kindling progresses, and that no significant variation in synchrony occurs along the septotemporal axis. It quantitatively demonstrates that the synchrony of neurons increases only along the lamellar axis during the process of epileptogenesis. It also indicates that the lamellar structure is an important concept in hippocampal synchrony analysis.

We developed a novel synchrony measurement, theta phase synchronization, by using theta oscillations as underlying clocks to investigate the synchrony of high frequency firing. Since the neurons do not fire periodically like chemical oscillators, for those who do not understand the underlying firing mechanism, the actual application of the phase model to neuronal firing data is vastly more difficult. Theta oscillations are essential for the normal functioning of the hippocampus and function as a clock for hippocampal activity during seizures [88-90]. Therefore, 
theta oscillations are the perfect underlying clocks for the new synchrony method. Theta phase synchronization revealed that the hippocampal CA1 neurons tend to fire around the negative peaks of the theta oscillations along the lamellar axis at the end of kindling, while having no preference at the beginning of kindling. However, the neuronal firings did not interact with the underlying theta oscillations in the septotemporal direction. No preferred firing phase was observed during kindling. The development of a preferred firing phase along the lamellar axis indicates that local neuronal firings became strongly influence by the slow theta oscillations. Additionally, the marked transition from unaffected to strongly affected indicates that the firings became more organized along the lamellar axis as kindling progressed. Also, as previously shown, theta phase synchronization successfully demonstrated that the synchrony level changes in the lamellar axis, but not in the septotemporal axis, during kindling. In conclusion, we presented a new approach to measure synchronization that is based on theta oscillations. This method gives an easy visualization of synchronization and firing patterns. Lastly, we gained insight into the temporal relationship between low frequency theta oscillations and high frequency firing during electrically evoked seizures. This approach is promising for the study of epileptic seizures, where synchronization is important and analysis of the firing pattern of neurons is needed.

Antiepileptic drugs are primarily intended to prevent epileptic seizures. To exhibit antiepileptic activity, a drug must act on one or more target molecules in the brain. These targets include ion channels, neurotransmitter transporters and neurotransmitter metabolic enzymes. The ultimate effect of these interactions is the modification of the bursting properties of neurons which is thought to reduce synchronization. Phenytoin obstructs recurrent action potentials by blocking voltage-dependent sodium channels $[20,94]$. Therefore, phenytoin should be able 
to reduce synchrony after treatment. LEV has multiple mechanisms of action $[97,98]$, though it has been shown to bind to the synaptic vesicle protein 2 (SV2) [99]. Loss of SV2 means that synaptic strength is decreased specifically during high frequency firing, like bursting activities [100]. Since levetiracetam inhibits synaptic excitation, it might also have an effect on synchrony. LEV has been shown in some in vitro models to decrease synchronization [113]. However, the changes in neuronal synchronization and propagation with AEDs treatment in awake animals have not been fully investigated and are poorly understood. In this study, we examined the effects of phenytoin and levetiracetam on synchrony and propagation during electrically evoked seizures in freely moving rats.

We found that synchrony decreases within the first 2-3 hours of the phenytoin or levetiracetam treatment before recovering to pre-injection levels, and the preferred firing phase value, which occurred around the trough of the theta oscillation, was lost after both treatments. This indicates that the synchrony level of neurons is reduced by phenytoin and levetiracetam. We also observed that both ADD and BSS were suppressed after the phenytoin and levetiracetam treatments. Similar results were already shown in the kindling model $[46,101,102]$. What is new is that the synchronization measurements showed that synchrony returned to pretreatment levels long before BSS or ADD. Therefore synchronization measurements may be a better indicator of seizure severity, and a superior measurement as to the effectiveness of a treatment, than ADD or BSS. As for seizure propagation, the results showed that the firing propagation pattern remained the same, which from proximal to distal through the transverse extent of the monitored CA1 cell layer, after both treatments. It suggests that both treatments do not affect the propagation pattern. In conclusion, phenytoin and levetiracetam attenuate the synchrony level as we expected, but do not affect the propagation pattern along the lamellar axis. 


\section{Chapter 8}

\section{Future Work}

\subsection{Neuronal Firing Patterns in the Dentate Gyrus and Hippocampal CA1 during Seizures}

Previous studies have been proposed that dentate granule cells act as a gate, regulating hippocampal excitation $[86,114]$. In contrast with pyramidal neurons, dentate granule cells generate single action potentials and do not generate bursts. They lack the recurrent connections necessary to synchronize and propagate bursts. The major input to the hippocampus is via the perforant path, arising from layer II of entorhinal cortex, innervating dentate gyrus (DG). Thus, within the normal hippocampus, pyramidal neurons can generate and propagate seizures while dentate granule cells resist their spread [115]. The dentate gyrus is hypothesized to act as a gate that filters the flow of information from the entorhinal cortex to the hippocampus $[115,116]$. The breakdown of this gating function of the dentate gyrus is considered crucial for the occurrence of seizures [53,117]. Voltage sensitive dye imaging technique is utilized to monitor function of the DG in slices prepared from epileptic animals, as entorhinal cortical afferents to the dentate gyrus are 
activated $[118,119]$. In vivo work shows that under normal conditions the resistance of the dentate gyrus to the development of epileptic discharges enables it to retard seizures. However, in vivo work also demonstrates that under appropriate conditions the dentate gyrus can support and sustain a robust and unique type of paroxysm (MDA)[120]. Data from these studies illustrated that in both nonepileptic and epileptic animals, these strongly inhibited dentate granule cells act as a gate, regulating hippocampal excitation. The relationship between the CA1 and DG firings during seizures would be helpful in investigating propagation pattern from the DG to CA1.

As mentioned before, recurrent seizures induce neuron loss and reorganization of neuronal circuitry with newly sprouted synapses. In the normal dentate gyrus, mossy fiber axons project a dense plexus into the hilus and extend a single, prominent axon into CA3. In temporal lobe epilepsy (TLE), the dentate mossy fiber axons undergo prominent sprouting and expand their terminal fields into the supragranular region of the inner molecular layer [121]. It is possible that the mossy fiber sprouting might alter the synchrony and propagation between the CA1 and DG. Studies have investigated CA1 pyramidal neuron density, pyramidal cell axonal morphology, location and types of sprouted mossy fibers [121, 122]. However, neuronal firing synchronization and propagation pattern in the hippocampal CA1 and DG in chronic epilepsy model have not been studied.

The future work could focus on using phase synchrony methods to analyze propagation and synchronization between the DG and the CA1 regions during electrically kindled seizures and recurrent spontaneous seizures of chronic epilepsy.

Two microelectrode arrays will be incorporated into the existing dynamic brain recording apparatus to allow multi-region simultaneous recordings in the hippocampus. Neuronal firing synchronization and the propagation patterns in the 
hippocampal CA1 and the DG will be studied by applying theta phase analysis and event synchronization. The propagation pattern from the DG to CA1 during spontaneous seizures of chronic epilepsy will be compared with that obtained in electrically kindled seizures. The effect of mossy fiber sprouting on synchrony and propagation between the CA1 and DG will also be investigated.

\subsection{Deep Brain Stimulation}

Antiepileptic drugs provide satisfactory control of seizures for most patients with epilepsy [18]. However, the development of rapid refractoriness to these drugs in status epilepticus (SE) [123] precludes successful management of the condition. It is estimated that about one third of patients do not respond favorably to currently available drug treatments and up to $50 \%$ experience major side effects of these treatments [124]. Currently, deep brain stimulation therapy is being tested as an alternative method to terminate seizures by de-synchronizing groups of neurons with an external electrical stimulus [125-128]. This experimental progress is greatly encouraging; however, it is not yet known what types of dynamic stimulation will be most effective in producing optimal results.

The current stimulation paradigm is based on a predetermined pulse-train stimulation waveform with a fixed frequency, amplitude and duration. This paradigm does not account for the complex relationship among individual neuronal behavior (waveform and response to stimulation), overall collective behavior, and degree of synchronization leading to seizure [2].

Based on what have been discovered in this study, building a precisely engineered stimulation paradigm will be promising. 


\section{Appendix A}

\section{MATLAB Code for EEG Data Analysis}

\section{A.1 Spike Detection}

1 function $[$ maxtab, mintab $]=$ peakdet $(\mathrm{v}, \operatorname{delta}, \mathrm{x})$

$\%$ PEAKDET find peaks in a time series

$\%[$ maxtab, mintab] $=$ peakdet $(v$, delta,$x)$ finds the local maxiam

$\%$ and minima in the vector $v$.

6

$\operatorname{maxtab}=[]$

$\operatorname{mintab}=[]$

$\mathrm{v}=\mathrm{v}(:) ; \%$ Just in case this wasn't a proper vector

11

$$
\begin{aligned}
& \text { if nargin }<3 \\
& \qquad x=(1 \text { :length }(\mathrm{v}))^{\prime} ; \\
& \text { else } \\
& \quad \mathrm{x}=\mathrm{x}(:)
\end{aligned}
$$




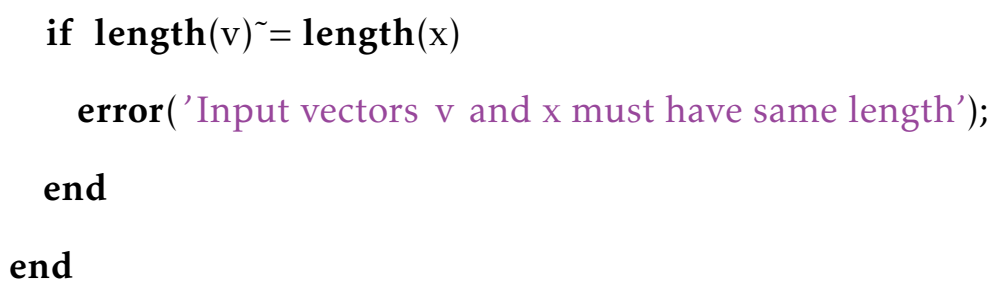

if lookformax

if this $<\mathrm{mx}$-delta

$$
\begin{aligned}
& \text { maxtab }=[\text { maxtab } ; \operatorname{mxpos} \mathrm{mx}] \\
& \mathrm{mn}=\text { this; } \operatorname{mnpos}=\mathrm{x}(\mathrm{i})
\end{aligned}
$$




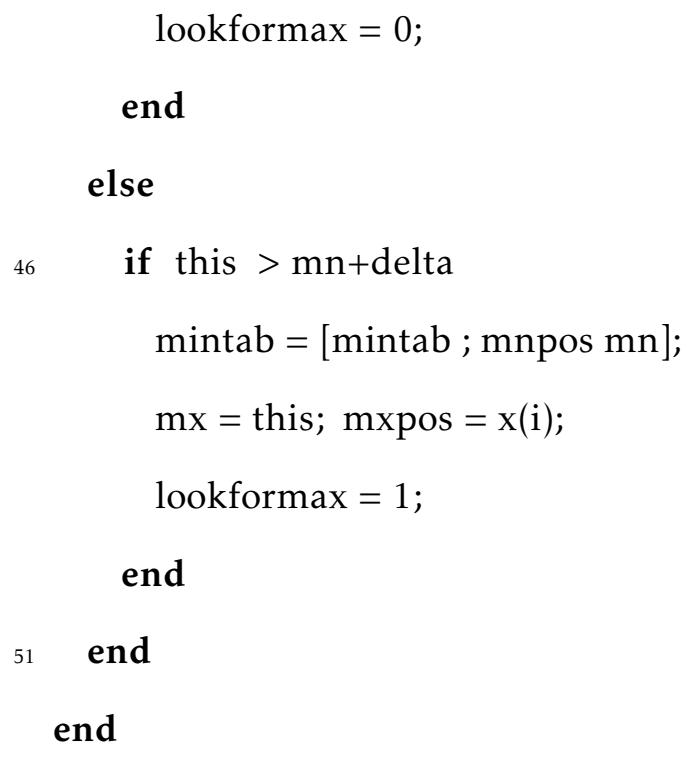

\section{A.2 Theta Phase Synchronization}

\section{A.2.1 Assign Theta Phase}

function [phase_data,phase_ch1,phase_ch2,phase_ch3,phase_ch4]=theta_dist(binWidth, new,theta_spikes_position,spikes_position_1,spikes_position_2,spikes_position_3, spikes_position_4)

$\%$ Assign theta phase value to all the peaks.

$3 \%$ new--filtered data

$\%$ theta_spikes_position --define theta cycle

\% spikes_position_1,2,3,4--all the peaks

phase_data $=[]$;

8 phase_data $(:, 1)=(1: \text { length }(\text { new }))^{\prime}$;

\%give each time point a phase value

for $\mathrm{k}=1$ :theta_spikes_position(1)

phase_data $(\mathrm{k}, 2)=2 *$ pi $/$ theta_spikes_position $(1) * \mathrm{k}$; 
end

13 for $i=1:($ length(theta_spikes_position) -1$)$

for $\mathrm{j}=$ theta_spikes_position(i) : theta_spikes_position $(\mathrm{i}+1)$

phase_data $(j, 2)=2 * \mathbf{p i} /($ theta_spikes_position $(\mathrm{i}+1)$-theta_spikes_position $(\mathrm{i})) *(\mathrm{j}-$

theta_spikes_position(i));

end

end

${ }_{18}$ for $\mathrm{z}=$ theta_spikes_position(length(theta_spikes_position)) :length(new)

phase_data $(z, 2)=2 *$ pi $/($ length $($ new $)$-theta_spikes_position(length(theta_spikes_position))

)$*(z$-theta_spikes_position(length(theta_spikes_position $)))$;

end

phase_ch1 = phase_data(spikes_position_1,2);

phase_ch2=phase_data(spikes_position_2,2);

23 phase_ch3=phase_data(spikes_position_3,2);

phase_ch4=phase_data(spikes_position_4,2);

\%plot theta phase distribution

subplot $(2,2,1)$

28 sort_phase_ch1 =sort $($ phase_ch 1$)$;

binCtrs $=0$ :binWidth: $2 \star \mathbf{p i}$;

counts1=hist(sort_phase_ch1,binCtrs);

bar(binCtrs, counts1/sum(counts1));

$\operatorname{xlim}\left(\left[\begin{array}{ll}0 & 2 * \mathbf{p i}\end{array}\right]\right)$;

$33 \operatorname{ylim}\left(\left[\begin{array}{ll}0 & 0.15\end{array}\right]\right)$;

$\operatorname{set}($ gca, 'FontSize', 20)

$\operatorname{set}($ gca, 'XTick', $[0$ pi $2 \star$ pi $]$ ) 


\section{$\operatorname{subplot}(2,2,2)$}

38 sort_phase_ch2=sort(phase_ch2);

binCtrs $=0$ :binWidth $: 2 * \mathbf{p i}$;

counts2=hist(sort_phase_ch2,binCtrs);

bar(binCtrs, counts2/sum(counts2));

$\operatorname{xlim}\left(\left[\begin{array}{ll}0 & 2 * \mathbf{p i}\end{array}\right]\right)$;

${ }_{43} \operatorname{ylim}\left(\left[\begin{array}{ll}0 & 0.15\end{array}\right]\right)$;

$\operatorname{set}($ gca, 'FontSize', 20)

$\operatorname{set}($ gca, 'XTick', [0 pi $2 *$ pi $]$ )

$\operatorname{subplot}(2,2,3)$

48 sort_phase_ch3=sort(phase_ch3);

binCtrs $=0$ :binWidth $: 2 * \mathbf{p i}$;

counts3=hist(sort_phase_ch3,binCtrs);

bar(binCtrs, counts3/sum(counts3));

$x \lim \left(\left[\begin{array}{ll}0 & 2 * \mathbf{p i}\end{array}\right]\right)$;

$53 \operatorname{ylim}\left(\left[\begin{array}{ll}0 & 0.15\end{array}\right]\right)$;

$\operatorname{set}($ gca, 'FontSize', 20)

$\operatorname{set}($ gca, 'XTick', [0 pi $2 *$ pi $]$ )

$\operatorname{subplot}(2,2,4)$

58 sort_phase_ch4=sort(phase_ch4);

binCtrs $=0$ :binWidth: $2 * \mathbf{p i}$;

counts4=hist(sort_phase_ch4,binCtrs);

bar(binCtrs, counts $4 /$ sum(counts 4$)$ );

$\mathbf{x l i m}\left(\left[\begin{array}{ll}0 & 2 * \mathbf{p i}\end{array}\right]\right)$;

${ }_{63} \operatorname{ylim}\left(\left[\begin{array}{ll}0 & 0.15\end{array}\right]\right)$; 
set (gca,' FontSize',20)

$\operatorname{set}\left(\right.$ gca, ${ }^{\prime} X$ Tick', $[0$ pi $2 *$ pi $]$ )

\section{A.2.2 Shannon Entropy}

function $[$ mean_1, mean_2, mean_3,mean_4] = mean_ShanEnt $($ phase_ch1,phase_ch2, phase_ch3,phase_ch4,window_size,slide,number_bins)

\% Calculate shannon entropy for each channel

$\%$ phase_ch1,2,3,4--theta phase value for all the peaks

$\%$ Shannon Entropy $=-\operatorname{sum}(P . * \log (P)) ; P--$ probability of each bin

5

ShanEnt $=[]$;

for $i=1$ :round $(($ length(phase_ch 1$)-$ window_size $) /$ slide)

sample_window $=$ phase $\_$ch $1(1+\operatorname{slide} *(i-1)$ : window_size $+\operatorname{slide} *(i-1))$;

$\mathrm{P}=$ hist(sample_window,number_bins)/window_size;

10 index_zeros $=$ find $(\mathrm{P}==0)$;

$\mathrm{P}($ index_zeros $)=[]$;

ShanEnt $(\mathrm{i})=-\operatorname{sum}(\mathrm{P} . \log (\mathrm{P}))$;

end

mean_1=mean(ShanEnt);

${ }_{15}$ ShanEnt=[];

for $i=1$ :round ((length(phase_ch2)-window_size)/slide)

sample_window $=$ phase_ch $2(1+\operatorname{slide} *(i-1):$ window_size + slide $*(i-1))$;

$\mathrm{P}=$ hist(sample_window,number_bins)/window_size;

20 index zeros $=$ find $(P==0)$;

$\mathrm{P}($ index zeros $)=[]$;

ShanEnt $(\mathrm{i})=-\operatorname{sum}(\mathrm{P} . \log (\mathrm{P}))$; 


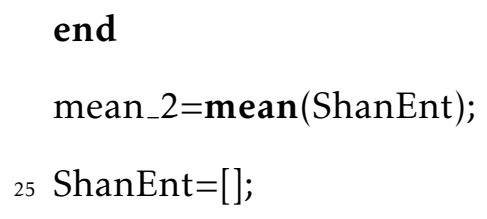

\section{A.3 Event Synchronization}

$\%$ Event Synchronization 
function $[$ sum_J] = event_synch(action_potentials_1,action_potentials_2, lag_index)

$4 \%$ action_potentials_1, action_potentials_2 --peaks in two time series

sum_J $_{-}=0 ; \%$ Initialize variable

for counter $=1$ :length(action_potentials_1) $\%$ Look at every action potential

$9 \%$ Verify that lag_index is smaller than half the interval between 2 action potentials

if counter $<=$ length(action_potentials__1) -1

$\min \_l a g=\min \left(\left[\operatorname{lag} \_\right.\right.$index,$\left(\right.$action_potentials_1 $(1$, counter +1$)-$ action $\_$potentials_1 $(1$, counter $)) / 2])$;

else

min_lag = lag_index;

14

end

\%Find a action potential of 2 that is between the action potential in 1 and the lag_index

ind $=$ find(action_potentials_2 $(1,:) \quad<=$ action_potentials_ $1(1$, counter $)+$ min_lag \& action_potentials_2 $(1,:)>$ action_potentials_1 (1,counter), 1$)$;

if isempty(ind)

19 $\%$ See if the action potentials in 1 and 2 have the same index ind $=$ find $($ action_potentials_2 $(1,:)==$ action_potentials_1 $(1$, counter $), 1)$; if isempty(ind) $\mathrm{J}=0 ; \%$ No action potential in 2 after action potential in 1 $\operatorname{sum}_{-} \mathrm{J}=\operatorname{sum}_{-} \mathrm{J}+\mathrm{J}$;

$24 \quad$ else

$\mathrm{J}=.5 ; \%$ Action potentials occur at the same time 
$\operatorname{sum}_{-} \mathrm{J}=\operatorname{sum}_{-} \mathrm{J}+\mathrm{J} ;$

end

else

$\%$ Recalulate lag index

if ind $>=2 \& \&$ counter $>=2 \& \&$ counter $\sim=$ length $\left(\right.$ action $\_$potentials $\left.\_1\right) \& \&$ ind $\sim=$

length(action_potentials_2)

$\min \_l a g=\min \left(\left[\operatorname{lag} \_\right.\right.$index,$($action_potentials_1 $(1$, counter +1$)-$ action_potentials_1(1,counter) $) / 2,($ action_potentials_2 $(1$, ind +1$)-$ action_potentials_2 $(1$, ind $)) / 2,($ action_potentials_1 $(1$, counter $)-$ action_potentials_1 $(1$, counter-1) $) / 2$,(action_potentials_2 $(1$,ind $)-$ action_potentials_2 $(1$, ind-1))/2]);

elseif ind $>=2 \& \&$ counter $==$ length $\left(\right.$ action_potentials $\left.\_1\right) \& \&$ ind $^{\sim}=$ length ( action_potentials_2)

$\min \_l a g=\min \left(\left[\operatorname{lag} \_\right.\right.$index,$($action_potentials_2 $(1$, ind +1$)-$ action_potentials_2 $(1$, ind $)) / 2,($ action_potentials_1 (1, counter)-action_potentials_1 1 ,counter $-1)) / 2,($ action_potentials_2 $(1$, ind $)-$ action_potentials_2 $(1$, ind -1$)) / 2])$; action_potentials_2)

$\min \_l a g=\min \left(\left[\operatorname{lag} \_\right.\right.$index,$($action_potentials_1 $(1$, counter +1$)-$ action_potentials_1(1,counter $)) / 2$,(action_potentials_1 (1, counter)action_potentials_1 $(1$, counter-1) $) / 2,($ action_potentials_2 $(1$,ind $)-$ action_potentials_2(1,ind-1))/2]);

elseif ind $<2 \& \&$ counter $<2$

$\min \_l a g=\min \left(\left[\operatorname{lag} \_\right.\right.$index,$($action_potentials_1 $(1$, counter +1$)-$ action_potentials_ $1(1$, counter $)) / 2$, (action_potentials_ $2(1$, ind +1$)-$ action_potentials_2(1,ind))/2]);

elseif ind $<2 \& \&$ counter $\sim$ length(action_potentials_1) 
$\min \_l a g=\min \left(\left[\operatorname{lag} \_\right.\right.$index,$($action_potentials_1 $(1$, counter +1$)-$ action_potentials_ $1(1$, counter $)) / 2,($ action_potentials_2 $2(1$, ind +1$)-$ action_potentials_2 $(1$,ind $)) / 2$,(action_potentials_1 $(1$, counter $)-$ action_potentials_1(1,counter-1))/2]);

elseif counter $<2 \& \&$ ind $^{\sim}=$ length(action_potentials_2)

$\min \_l a g=\min ([$ lag_index,$($ action_potentials_1 $(1$, counter +1$)-$ action_potentials_1 $(1$, counter $)) / 2,($ action_potentials_2 $(1$, ind +1$)-$ action_potentials_2 $(1$, ind $)) / 2$, (action_potentials_2 $(1$, ind $)-$ action_potentials_2(1,ind-1))/2]);

elseif counter $==$ length(action_potentials_1) \& \& ind==length( action_potentials_2) $\min \_l a g=\min \left(\left[\operatorname{lag} \_\right.\right.$index,$($action_potentials_1 $(1$, counter $)-$ action_potentials_1 $(1$, counter-1) $) / 2,($ action_potentials_2 $(1$,ind $)-$ action_potentials_2(1,ind-1))/2]);

$\min \_l a g=\min \left(\left[\operatorname{lag} \_\right.\right.$index,$($action_potentials_1 $(1$, counter +1$)-$ action_potentials_1 1 , counter $)) / 2$, (action_potentials_2 $(1$,ind $)-$ action_potentials_2(1,ind-1))/2]);

else \%counter at end and ind $<2$

$\min \_l a g=\min \left(\left[\operatorname{lag} \_\right.\right.$index,$($action_potentials_2 $(1$, ind +1$)-$ action_potentials_2 $(1$, ind $)) / 2,($ action_potentials_1 (1, counter)-action_potentials_1 1 ,counter $-1)) / 2])$;

end

\%Find a action potential of 2 that is between the action potential in 1 and the lag_index ind $=$ find $($ action_potentials_2 $(1,:)<=$ action_potentials_ $1(1$, counter $)+$ min_lag 
$\&$ action_potentials_2 $2(1,:)>$ action_potentials_1 (1, counter), 1);

if isempty(ind)

$\mathrm{J}=0 ; \%$ No action potential in 2 after action potential in 1

54 $\operatorname{sum}_{-} \mathrm{J}=\operatorname{sum}_{-} \mathrm{J}+\mathrm{J}$;

else

$\mathrm{J}=1$; \%Action potential in 2 is within the lag_index of 1 $\operatorname{sum}_{-} \mathrm{J}=\operatorname{sum}_{-} \mathrm{J}+\mathrm{J}$;

end

$59 \quad$ end

end 


\section{Appendix B}

\section{List of Publications Based on the Dissertation}

1. Xin Ren, J.L. Hudson and J. Kapur, "Dynamic Emergence of Neuronal Synchrony during seizures" J. Physiol., (in preparation). 


\section{Bibliography}

[1] P. Carlen, M. Derchansky, D. Rokni, J. T. Rick, R. Wennberg, B. L. Bardakjian, L. Zhang, and Y. Yarom. Bidirectional multisite seizure propagation in the intact isolated hippocampus: The multifocality of the seizure "focus". Neurobiol Dis., 23:312-328, 2006.

[2] M. G. Frei, H. P. Zaveri, S. Arthurs, G. K. Bergey, C. C. Jouny, K. Lehnertz, J. Gotman, I. Osorio, T. I. Netoff, W. J. Freeman, J. Jefferys, G. Worrell, V. Quyen Mle, S. J. Schiff, and F. Mormann. Controversies in epilepsy: debates held during the fourth international workshop on seizure prediction. Epilepsy Behav, 19:4-16, 2010.

[3] A. Pitkanen, P.A Schwartzkroin, and S.L. MoshA. Models of seizures and epilepsy. Elsevier Academic, 2006.

[4] D. G. Amaral and M. P. Witter. The three-dimensional organization of the hippocampal formation: a review of anatomical data. Neuroscience, $31: 571-91,1989$.

[5] A. B. Kibler and D. M. Durand. Orthogonal wave propagation of epileptiformactivity in the planar mouse hippocampus in vitro. Epilepsia, 52: 1590-1600, 2011. 
[6] D. Jaeger, S. Gilman, and J. W. Aldridge. A multiwire microelectrode for single unit recording in deep brain structures. J Neurosci Methods, 32:143-8, 1990.

[7] D. Lee. Analysis of phase-locked oscillations in multi-channel singleunit spike activity with wavelet cross-spectrum. journal of Neuroscience Methods, 115:67-75, 2002.

[8] X. Li, D. Cui, P. Jiruska, J. E. Fox, X. Yao, and J. G. Jefferys. Synchronization measurement of multiple neuronal populations. J Neurophysiol, 98: 3341-8, 2007.

[9] P. P. Lenck-Santini and G. L. Holmes. Altered phase precession and compression of temporal sequences by place cells in epileptic rats. $J$ Neurosci, 28:5053-62, 2008.

[10] G. V. Goddard. The kindling model of epilepsy. Trends in Neurosciences, 6:275-279, 1983.

[11] Jaideep Kapur. A loss of GABA-mediated inhibition contributes to the self-reinforcement of kindled seizures. PhD thesis, University of Virginia, 1988.

[12] D. A. Henze, Z. Borhegyi, J. Csicsvari, A. Mamiya, K. D. Harris, and G. Buzsaki. Intracellular features predicted by extracellular recordings in the hippocampus in vivo. J Neurophysiol, 84:390-400, 2000.

[13] J. Y. Chang, L. H. Shi, F. Luo, D. J. Woodward, and D. C. McIntyre. Temporal sequence of ictal discharges propagation in the corticolimbic basal ganglia system during amygdala kindled seizures in freely moving rats. Epilepsy Research, 73:85-97, 2007. 
[14] H. Osterhage, F. Mormann, M. Staniek, and K. Lehnertz. Measuring synchronization in the epileptic brain: A comparison of different approaches. International journal of Bifurcation and Chaos, 17:3539-3544, 2007.

[15] A. Wilmer, M. H. de Lussanet, and M. Lappe. A method for the estimation of functional brain connectivity from time-series data. $\operatorname{Cog} n$ Neurodyn, 4:133-49, 2010.

[16] S. J. Schiff. Dangerous phase. Neuroinformatics, 3:315-317, 2005.

[17] R. Quian Quiroga, T. Kreuz, and P. Grassberger. Event synchronization: a simple and fast method to measure synchronicity and time delay patterns. Phys Rev E Stat Nonlin Soft Matter Phys, 66:041904, 2002.

[18] M. A. Rogawski and W. Loscher. The neurobiology of antiepileptic drugs. Nat Rev Neurosci, 5:553-64, 2004.

[19] S. C. Schachter. Antiepileptic drug therapy: General treatment principles and application for special patient populations. Epilepsia, 40: S20-S25, 1999.

[20] R. L. Macdonald and K. M. Kelly. Antiepileptic drug mechanisms of action. Epilepsia, 36:S2-S12, 1995.

[21] Raymond P. Kesner. Neurobiology of learning and memory. Academic Press, 1998.

[22] D. A. McCormick and D. Contreras. On the cellular and network bases of epileptic seizures. Annual Review of Physiology, 63:815-846, 2001. 
[23] R. J. Delorenzo, J. M. Pellock, A. R. Towne, and J. G. Boggs. Epidemiology of status epilepticus. journal of Clinical Neurophysiology, 12:316-325, 1995.

[24] R. K. Wong, R. D. Traub, and R. Miles. Cellular basis of neuronal synchrony in epilepsy. Adv Neurol, 44:583-92, 1986.

[25] K. J. Staley, J. S. Bains, and J. M. Longacher. Reciprocal interactions between ca3 network activity and strength of recurrent collateral synapses. Nature Neuroscience, 2:720-726, 1999.

[26] L. D. Iasemidis, P. M. Pardalos, D. S. Shiau, W. Chaovalitwongse, K. Narayanan, S. Kumar, P. R. Carney, and J. C. Sackellares. Prediction of human epileptic seizures based on optimization and phase changes of brain electrical activity. Optimization Methods E Software, 18:81-104, 2003.

[27] L. D. Iasemidis, D. S. Shiau, J. C. Sackellares, P. M. Pardalos, and A. Prasad. Dynamical resetting of the human brain at epileptic seizures: application of nonlinear dynamics and global optimization techniques. IEEE Trans Biomed Eng, 51:493-506, 2004.

[28] K. Lehnertz. Non-linear time series analysis of intracranial eeg recordings in patients with epilepsy-an overview. Int J Psychophysiol, 34:45-52, 1999.

[29] M. Le Van Quyen, J. Soss, V. Navarro, R. Robertson, M. Chavez, M. Baulac, and J. Martinerie. Preictal state identification by synchronization changes in long-term intracranial eeg recordings. Clinical Neurophysiology, 116:559-568, 2005. 
[30] B. Litt, R. Esteller, J. Echauz, M. D’Alessandro, R. Shor, T. Henry, P. Pennell, C. Epstein, R. Bakay, M. Dichter, and G. Vachtsevanos. Epileptic seizures may begin hours in advance of clinical onset: A report of five patients. Neuron, 30:51-64, 2001.

[31] P. M. Pardalos, V. Yatsenko, J. C. Sackellares, D. S. Shiau, W. Chaovalitwongse, and L. D. Iasemidis. Analysis of eeg data using optimization, statistics, and dynamical system techniques. Computational Statistics $\mathcal{E}$ Data Analysis, 44:391-408, 2003.

[32] M. R. Sperling and M. J. O'Connor. Comparison of depth and subdural electrodes in recording temporal lobe seizures. Neurology, 39:1497-504, 1989.

[33] H.O. Luders and Y. G. Comair. Epilepsy Surgery. Lippincott Williams and Wilkins, 2000.

[34] N. So, P. Gloor, L. F. Quesney, M. Jones-Gotman, A. Olivier, and F. Andermann. Depth electrode investigations in patients with bitemporal epileptiform abnormalities. Annals of Neurology, 25:423-31, 1989.

[35] K. L. Drake, K. D. Wise, J. Farraye, D. J. Anderson, and S. L. Bement. Performance of planar multisite microprobes in recording extracellular single-unit intracortical activity. Ieee Transactions on Biomedical Engineering, 35:719-732, 1988.

[36] A. B. Kibler, B. G. Jamieson, and D. M. Durand. A high aspect ratio microelectrode array for mapping neural activity in vitro. Journal of Neuroscience Methods, 204:296-305, 2012. 
[37] A. C. Hoogerwerf and K. D. Wise. A three-dimensional microelectrode array for chronic neural recording. IEEE Trans Biomed Eng, 41:1136-46, 1994.

[38] A. Bragin, J. Csicsvari, M. Penttonen, and G. Buzsaki. Epileptic afterdischarge in the hippocampal-entorhinal system: current source density and unit studies. Neuroscience, 76:1187-203, 1997.

[39] A. Bragin, M. Penttonen, and G. Buzsaki. Termination of epileptic afterdischarge in the hippocampus. J Neurosci, 17:2567-79, 1997.

[40] M. S. Fee, P. P. Mitra, and D. Kleinfeld. Automatic sorting of multiple unit neuronal signals in the presence of anisotropic and non-gaussian variability. J Neurosci Methods, 69:175-88, 1996.

[41] J. C. Sanchez, T. H. Mareci, W. M. Norman, J. C. Principe, W. L. Ditto, and P. R. Carney. Evolving into epilepsy: Multiscale electrophysiological analysis and imaging in an animal model. Experimental Neurology, 198: 31-47, 2006.

[42] H. B. Michelson and E. W. Lothman. An ontogenetic study of kindling using rapidly recurring hippocampal seizures. Brain Res Dev Brain Res, 61:79-85, 1991.

[43] E. W. Lothman and J. M. Williamson. Closely spaced recurrent hippocampal seizures elicit two types of heightened epileptogenesis: a rapidly developing, transient kindling and a slowly developing, enduring kindling. Brain Res, 649:71-84, 1994.

[44] E. H. Bertram and J. F. Cornett. The evolution of a rat model of chronic spontaneous limbic seizures. Brain Res, 661:157-62, 1994. 
[45] U. Sayin, S. Osting, J. Hagen, P. Rutecki, and T. Sutula. Spontaneous seizures and loss of axo-axonic and axo-somatic inhibition induced by repeated brief seizures in kindled rats. J Neurosci, 23:2759-68, 2003.

[46] E. W. Lothman, J. M. Williamson, and K. E. VanLandingham. Intraperitoneal phenytoin suppresses kindled responses: effects on motor and electrographic seizures. Epilepsy Res, 9:11-8, 1991.

[47] J. Tuunanen and A. Pitkanen. Do seizures cause neuronal damage in rat amygdala kindling? Epilepsy Res, 39:171-6, 2000.

[48] T. Sutula, S. Lauersdorf, M. Lynch, C. Jurgella, and A. Woodard. Deficits in radial arm maze performance in kindled rats: evidence for longlasting memory dysfunction induced by repeated brief seizures. $J$ Neurosci, 15:8295-301, 1995.

[49] S. K. Schultz. Principles of neural science, 4th edition. American journal of Psychiatry, 158:662-662, 2001.

[50] P. Andersen, T. V. P. Bliss, and K. K. Skrede. Lamellar organization of hippocampal excitatory pathways. Experimental Brain Research, 13:222, 1971.

[51] X. G. Li, P. Somogyi, A. Ylinen, and G. Buzsaki. The hippocampal ca3 network: an in vivo intracellular labeling study. J Comp Neurol, 339: 181-208, 1994.

[52] P. Andersen, A. F. Soleng, and M. Raastad. The hippocampal lamella hypothesis revisited. Brain Research, 886:165-171, 2000. 
[53] I. Mody, J. Behr, and K. J. Lyson. Enhanced propagation of epileptiform activity through the kindled dentate gyrus. journal of Neurophysiology, 79:1726-1732, 1998.

[54] C. A. Schevon, S. A. Weiss, Jr. McKhann, G., R. R. Goodman, R. Yuste, R. G. Emerson, and A. J. Trevelyan. Evidence of an inhibitory restraint of seizure activity in humans. Nat Commun, 3:1060, 2012.

[55] George Paxinos. The rat nervous system. Academic Press, 1995.

[56] Wilder Penfield and Herbert H. Jasper. Epilepsy and the functional anatomy of the human brain. Little Brown, 1954.

[57] D. G. Margineanu. Epileptic hypersynchrony revisited. Neuroreport, 21: 963-7, 2010.

[58] A. Cymerblit-Sabba and Y. Schiller. Development of hypersynchrony in the cortical network during chemoconvulsant-induced epileptic seizures in vivo. J Neurophysiol, 107:1718-30, 2012.

[59] L. Chocholova, J. Faber, and Z. Taichmanova. Correlation of spike intervals and amplitudes in hypersynchronous eeg episodes in rat with cobalt focus. Act Nerv Super (Praha), 17:134-8, 1975.

[60] M. Chavez, M. L. Quyen, Q. Navarro, M. Baulac, and J. Martinerie. Spatio-temporal dynamics prior to neocortical seizures: Amplitude versus phase couplings. Ieee Transactions on Biomedical Engineering, 50: 571-583, 2003.

[61] M. Le Van Quyen, J. Martinerie, V. Navarro, M. Baulac, and F. J. Varela. 
Characterizing neurodynamic changes before seizures. Journal of Clinical Neurophysiology, 18:191-208, 2001.

[62] F. Mormann, T. Kreuz, R. G. Andrzejak, P. David, K. Lehnertz, and C. E. Elger. Epileptic seizures are preceded by a decrease in synchronization. Epilepsy Research, 53:173-185, 2003.

[63] T. I. Netoff and S. J. Schiff. Decreased neuronal synchronization during experimental seizures. J Neurosci, 22:7297-307, 2002.

[64] S. J. Schiff, T. Sauer, R. Kumar, and S. L. Weinstein. Neuronal spatiotemporal pattern discrimination: The dynamical evolution of seizures. Neuroimage, 28:1043-1055, 2005.

[65] P. Jiruska, M. de Curtis, J. G. Jefferys, C. A. Schevon, S. J. Schiff, and K. Schindler. Synchronization and desynchronization in epilepsy: controversies and hypotheses. J Physiol, 591:787-97, 2013.

[66] A. Adhikari, T. Sigurdsson, M. A. Topiwala, and J. A. Gordon. Crosscorrelation of instantaneous amplitudes of field potential oscillations: A straightforward method to estimate the directionality and lag between brain areas. Epilepsy Research, 191:191-200, 2010.

[67] J. Arnhold, P. Grassberger, K. Lehnertz, and C. E. Elger. A robust method for detecting interdependences: application to intracranially recorded eeg. Physica D-Nonlinear Phenomena, 134:419-430, 1999.

[68] J. P. Lachaux, E. Rodriguez, J. Martinerie, and F. J. Varela. Measuring phase synchrony in brain signals. Human Brain Mapping, 8:194-208, 1999. 
[69] K. Lehnertz, R. G. Andrzejak, J. Arnhold, T. Kreuz, F. Mormann, C. Rieke, G. Widman, and C. E. Elger. Nonlinear eeg analysis in epilepsy: Its possible use for interictal focus localization, seizure anticipation, and prevention. Physica D-Nonlinear Phenomena, 18:209-222, 2001.

[70] M. Le Van Quyen, J. Martinerie, M. Baulac, and F. Varela. Anticipating epileptic seizures in real time by a non-linear analysis of similarity between eeg recordings. Neuroreport, 10:2149-2155, 1999.

[71] E. Rodriguez, N. George, J. P. Lachaux, J. Martinerie, B. Renault, and F. J. Varela. Perception's shadow: long-distance synchronization of human brain activity. Nature, 397:430-433, 1999.

[72] M. G. Rosenblum, A. S. Pikovsky, and J. Kurths. Phase synchronization of chaotic oscillators. Physical Review Letters, 76:1804-1807, 1996.

[73] S. J. Schiff, P. So, T. Chang, R. E. Burke, and T. Sauer. Detecting dynamical interdependence and generalized synchrony through mutual prediction in a neural ensemble. Physical Review E, 54:6708-6724, 1996.

[74] P. Tass, M. G. Rosenblum, J. Weule, J. Kurths, A. Pikovsky, J. Volkmann, A. Schnitzler, and H. J. Freund. Detection of n:m phase locking from noisy data: Application to magnetoencephalography. Physical Review Letters, 81:3291-3294, 1998.

[75] F. Varela, J. P. Lachaux, E. Rodriguez, and J. Martinerie. The brainweb: Phase synchronization and large-scale integration. Nature Reviews Neuroscience, 2:229-239, 2001.

[76] N. F. Rulkov, M. M. Sushchik, L. S. Tsimring, and H. D. I. Abarbanel. 
Generalized synchronization of chaos in directionally coupled chaotic systems. Physical Review E, 51:980-994, 1995.

[77] M. Le van Quyen, J. Martinerie, C. Adam, and F. J. Varela. Nonlinear analyses of interictal eeg map the brain interdependences in human focal epilepsy. Journal of Clinical Neurophysiology, 127:250-266, 1999.

[78] K. M. Bertashius. Propagation of human complex-partial seizures - a correlation-analysis. Electroencephalography and Clinical Neurophysiology, 78:333-340, 1991.

[79] P. Jiruska, M. Tomasek, D. Netuka, J. Otahal, J. G. R. Jefferys, X. L. Li, and P. Marusic. Clinical impact of a high-frequency seizure onset zone in a case of bitemporal epilepsy. Epileptic Disorders, 10:231-238, 2008.

[80] M. Muller, G. Baier, C. Rummel, and K. Schindler. Estimating the strength of genuine and random correlations in non-stationary multivariate time series. Epl, 84, 2008.

[81] K. Schindler, H. Leung, C. E. Elger, and K. Lehnertz. Assessing seizure dynamics by analysing the correlation structure of multichannel intracranial eeg. Brain, 130:65-77, 2007.

[82] F. Wendling, F. Bartolomei, J. J. Bellanger, J. Bourien, and P. Chauvel. Epileptic fast intracerebral eeg activity: evidence for spatial decorrelation at seizure onset. Brain, 126:1449-1459, 2003.

[83] G. Ruhenstroth-Bauer, S. Vogl, H. Baumer, C. Moritz, and H. M. Weinmann. Natural atmospherics and occurrence of seizures in six adolescents with epilepsy: a cross correlation study. Seizure, 4:303-6, 1995. 
[84] M. F. Muller, G. Baier, Y. L. Jimenez, A. O. M. Garcia, C. Rummel, and K. Schindler. Evolution of genuine cross-correlation strength of focal onset seizures. Journal of Clinical Neurophysiology, 28:450-462, 2011.

[85] Y. Mizuno-Matsumoto, K. Okazaki, A. Kato, T. Yoshimine, Y. Sato, S. Tamura, and T. Hayakawa. Visualization of epileptogenic phenomena using cross-correlation analysis: Localization of epileptic foci and propagation of epileptiform discharges. Ieee Transactions on Biomedical Engineering, 46:271-279, 1999.

[86] I. Z. Kiss, M. Quigg, S. H. Chun, H. Kori, and J. L. Hudson. Characterization of synchronization in interacting groups of oscillators: application to seizures. Biophys J, 94:1121-30, 2008.

[87] E. V. Lubenov and A. G. Siapas. Hippocampal theta oscillations are travelling waves. Nature, 459:534-9, 2009.

[88] J. O'Keefe and J. Dostrovsky. The hippocampus as a spatial map. preliminary evidence from unit activity in the freely-moving rat. Brain Res, 34:171-5, 1971.

[89] J. O'Keefe and D. H. Conway. Hippocampal place units in the freely moving rat: why they fire where they fire. Exp Brain Res, 31:573-90, 1978.

[90] J. O'Keefe and M. L. Recce. Phase relationship between hippocampal place units and the eeg theta rhythm. Hippocampus, 3:317-30, 1993.

[91] A. G. Siapas, E. V. Lubenov, and M. A. Wilson. Prefrontal phase locking to hippocampal theta oscillations. Neuron, 46:141-151, 2005. 
[92] C. Geisler, K. Diba, E. Pastalkova, K. Mizuseki, S. Royer, and G. Buzsaki. Temporal delays among place cells determine the frequency of population theta oscillations in the hippocampus. Proceedings of the National Academy of Sciences of the United States of America, 107:7957-7962, 2010.

[93] Putnam Tj Merritt H. A new series of anticonvulsant drugs tested by experiments on animals. Archives of Neurology And Psychiatry, 39: 1003-1015, 1938.

[94] Y. Yaari, M. E. Selzer, and J. H. Pincus. Phenytoin: mechanisms of its anticonvulsant action. Annals of Neurology, 20:171-84, 1986.

[95] S. D. Shorvon, A. Lowenthal, D. Janz, E. Bielen, and P. Loiseau. Multicenter double-blind, randomized, placebo-controlled trial of levetiracetam as add-on therapy in patients with refractory partial seizures. european levetiracetam study group. Epilepsia, 41:1179-86, 2000.

[96] J. J. Cereghino, V. Biton, B. Abou-Khalil, F. Dreifuss, L. J. Gauer, and I. Leppik. Levetiracetam for partial seizures: results of a double-blind, randomized clinical trial. Neurology, 55:236-42, 2000.

[97] C. Y. Lee, C. C. Chen, and H. H. Liou. Levetiracetam inhibits glutamate transmission through presynaptic p/q-type calcium channels on the granule cells of the dentate gyrus. British journal of Pharmacology, 158: 1753-1762, 2009.

[98] M. Madeja, D. G. Margineanu, A. Gorji, E. Siep, P. Boerrigter, H. Klitgaard, and E. J. Speckmann. Reduction of voltage-operated potassium currents by levetiracetam: a novel antiepileptic mechanism of action? Neuropharmacology, 45:661-71, 2003. 
[99] B. A. Lynch, N. Lambeng, K. Nocka, P. Kensel-Hammes, S. M. Bajjalieh, A. Matagne, and B. Fuks. The synaptic vesicle protein sv2a is the binding site for the antiepileptic drug levetiracetam. Proceedings of the National Academy of Sciences of the United States of America, 101: 9861-9866, 2004.

[100] K. L. Custer, N. S. Austin, J. M. Sullivan, and S. M. Bajjalieh. Synaptic vesicle protein 2 enhances release probability at quiescent synapses. journal of Neuroscience, 26:1303-1313, 2006.

[101] S. Tokuda, N. Sofue, Y. Ohno, M. Sasa, and T. Serikawa. Inhibitory effects of levetiracetam on absence seizures in a novel absence-like epilepsy animal model, groggy rat. Brain Research, 1359:298-303, 2010.

[102] W. Loscher, D. Honack, and C. Rundfeldt. Antiepileptogenic effects of the novel anticonvulsant levetiracetam (ucb 1059) in the kindling model of temporal lobe epilepsy. journal of Pharmacology and Experimental Therapeutics, 284:474-479, 1998.

[103] G. Renfrey, H. Schlinger, J. Jakubow, and A. Poling. Effects of phenytoin and phenobarbital on schedule-controlled responding and seizure activity in the amygdala-kindled rat. journal of Pharmacology and Experimental Therapeutics, 248:967-973, 1989.

[104] R. J. Racine. Modification of seizure activity by electrical stimulation. ii. motor seizure. Electroencephalogr Clin Neurophysiol, 32:281-94, 1972.

[105] G. Buzsaki. Theta oscillations in the hippocampus. Neuron, 33:325-40, 2002. 
[106] V. S. Sohal, S. Pangratz-Fuehrer, U. Rudolph, and J. R. Huguenard. Intrinsic and synaptic dynamics interact to generate emergent patterns of rhythmic bursting in thalamocortical neurons. J Neurosci, 26:4247-55, 2006.

[107] gliageek. Illustration of a hippocampal neuron. frontalcortex.com, 2009. URL http://frontalcortex .com/?page=oll $\mid$ \&topic= $24 \backslash \& q i d=1223$.

[108] R. Brette and A. Destexhe. Handbook of Neural Activity Measurement. Cambridge University Press, 2012.

[109] T. L. Richardson, R. W. Turner, and J. J. Miller. Action-potential discharge in hippocampal ca1 pyramidal neurons - current source-density analysis. Journal of Neurophysiology, 58:981-996, 1987.

[110] P. Andersen, R. Morris, D. Amaral, T. Bliss, and John O'Keef. The Hippocampus Book. Oxford University Press, USA, 2006.

[111] Ivan Osorio and Ying-Cheng Lai. A phase-synchronization and randommatrix based approach to multichannel time-series analysis with application to epilepsy. Chaos, 21:033108, 2011.

[112] A. Dennison, G. C. Teskey, and D. P. Cain. Persistence of kindling: effect of partial kindling, retention interval, kindling site, and stimulation parameter. J Neurosci, 21:172-182, 1995.

[113] I. Niespodziany, H. Klitgaard, and D. G. Margineanu. Desynchronizing effect of levetiracetam on epileptiform responses in rat hippocampal slices. Neuroreport, 14(9):1273-1276, 2003. 
[114] C. W. Ang, G. C. Carlson, and D. A. Coulter. Hippocampal ca1 circuitry dynamically gates direct cortical inputs preferentially at theta frequencies. J Neurosci, 25:9567-80, 2005.

[115] E. W. Lothman, J. L. Stringer, and E. H. Bertram. The dentate gyrus as a control point for seizures in the hippocampus and beyond. Epilepsy Research, 7:301-313, 1992.

[116] U. Heinemann, H. Beck, J. P. Dreier, E. Ficker, J. Stabel, and C. L. Zhang. The dentate gyrus as a regulated gate for the propagation of epileptiform activity. Epilepsy Research, 7:273-280, 1992.

[117] C. R. Houser and Z. C. Peng. Temporal patterns of fos expression in the dentate gyrus after spontaneous seizures in a mouse model of temporal lobe epilepsy. journal of Neuroscience, 25:7210-7220, 2005.

[118] H. R. Pathak, F. Weissinger, M. Terunuma, G. C. Carlson, F. C. Hsu, S. J. Moss, and D. A. Coulter. Disrupted dentate granule cell chloride regulation enhances synaptic excitability during development of temporal lobe epilepsy. J Neurosci, 27:14012-22, 2007.

[119] C. W. Ang, G. C. Carlson, and D. A. Coulter. Massive and specific dysregulation of direct cortical input to the hippocampus in temporal lobe epilepsy. J Neurosci, 26:11850-6, 2006.

[120] E. W. Lothman. Functional anatomy: a challenge for the decade of the brain. Epilepsia, 32 Suppl 5:S3-13, 1991.

[121] J. E. Cavazos, P. Zhang, R. Qazi, and T. P. Sutula. Ultrastructural features of sprouted mossy fiber synapses in kindled and kainic acid-treated rats. J Comp Neurol, 458:272-92, 2003. 
[122] B. N. Smith and F. E. Dudek. Short- and long-term changes in ca1 network excitability after kainate treatment in rats. J Neurophysiol, 85: $1-9,2001$.

[123] J. Kapur and R. L. Macdonald. Rapid seizure-induced reduction of benzodiazepine and $\mathrm{zn} 2+$ sensitivity of hippocampal dentate granule cell gabaa receptors. J Neurosci, 17:7532-40, 1997.

[124] Y. Li and D. J. Mogul. Electrical control of epileptic seizures. journal of Clinical Neurophysiology, 24:197-204, 2007.

[125] B. J. Gluckman, H. Nguyen, S. L. Weinstein, and S. J. Schiff. Adaptive electric field control of epileptic seizures. J Neurosci, 21:590-600, 2001.

[126] W. H. Theodore and R. S. Fisher. Brain stimulation for epilepsy. Lancet Neurology, 3:111-118, 2004.

[127] M. E. Colpan, Y. Li, J. Dwyer, and D. J. Mogul. Proportional feedback stimulation for seizure control in rats. Epilepsia, 48:1594-603, 2007.

[128] R. Fisher, V. Salanova, T. Witt, R. Worth, T. Henry, R. Gross, K. Oommen, I. Osorio, J. Nazzaro, D. Labar, M. Kaplitt, M. Sperling, E. Sandok, J. Neal, A. Handforth, J. Stern, A. DeSalles, S. Chung, A. Shetter, D. Bergen, R. Bakay, J. Henderson, J. French, G. Baltuch, W. Rosenfeld, A. Youkilis, W. Marks, P. Garcia, N. Barbaro, N. Fountain, C. Bazil, R. Goodman, G. McKhann, K. Babu Krishnamurthy, S. Papavassiliou, C. Epstein, J. Pollard, L. Tonder, J. Grebin, R. Coffey, and N. Graves. Electrical stimulation of the anterior nucleus of thalamus for treatment of refractory epilepsy. Epilepsia, 51:899-908, 2010. 